\title{
GEOMETRIC STRUCTURES OF THE CLASSICAL GENERAL RELATIVISTIC PHASE SPACE
}

\author{
JOSEF JANYŠKA AND MARCO MODUGNO
}

\begin{abstract}
This paper is concerned with basic geometric properties of the phase space of a classical general relativistic particle, regarded as the 1st jet space of motions, i.e. as the 1st jet space of timelike 1-dimensional submanifolds of spacetime. This setting allows us to skip constraints.

Our main goal is to determine the geometric conditions by which the Lorentz metric and a connection of the phase space yield contact and Jacobi structures. In particular, we specialise these conditions to the cases when the connection of the phase space is generated by the metric and an additional tensor. Indeed, the case generated by the metric and the electromagnetic field is included, as well.
\end{abstract}

\section{Contents}

1. Introduction 2

2. Geometry of spacetime 5

2.1. Spacetime 5

2.2. Spacetime connections $\quad 5$

2.3. Differential operators 7

2.4. Spacetime 2-forms and 2-vectors 8

3. Geometric structures of the tangent bundle 9

3.1. General spacetime connection case 9

3.2. Linear spacetime connection case $\quad 10$

3.3. Non-metric spacetime connection case $\quad 10$

4. Geometry of the Einstein phase space $\quad 12$

4.1. Jets of submanifolds 12

4.2. Phase space $\quad 13$

4.3. Orthogonal splittings $\quad 13$

4.4. Splitting of the spacetime 2-form 15

4.5. Vertical bundle of the phase space $\quad 16$

4.6. Phase connection 17

4.7. Dynamical phase connection 17

2000 Mathematics Subject Classification. 53B15, 53B30, 53B50, 53D05, 53D17, 58A10, 58A20, 58A32.

Key words and phrases. Spacetime, phase space, spacetime connection, phase connection, Schouten bracket, Frölicher-Nijenhuis bracket, symplectic structure, Poisson structure, Jacobi structure.

This research has been supported by the Ministry of Education of the Czech Republic under the project MSM0021622409, by the Grant agency of the Czech Republic under the project GA 201/05/0523, by MIUR of Italy under the project PRIN 2005 "Simmetrie e Supersimmetrie Classiche e Quantistiche", by GNFM of INdAM and by Florence University. 
4.8. Phase 2-form and 2-vector

4.9. Spacetime and phase 2-forms and 2-vectors $\quad 19$

5. Contact and Jacobi structures: general case $\quad 21$

5.1. Regularity and duality $\quad 21$

5.2. Splittings of the phase tangent and cotangent bundles 23

5.3. Almost-cosymplectic-contact structure $\quad 26$

5.4. Almost-coPoisson-Jacobi structure $\quad 28$

5.5. Contact structure $\quad 34$

5.6. Jacobi structure $\quad 35$

6. Contact and Jacobi structures: linear case 38

6.1. Covariant derivatives and the adapted base $\quad 38$

6.2. Almost-cosymplectic-contact structure $\quad 43$

6.3. Almost-coPoisson-Jacobi structure $\quad 44$

6.4. Contact structure $\quad 46$

6.5. Jacobi structure $\quad 47$

7. Metric and non-metric phase objects 47

7.1. Metric contact and Jacobi structures $\quad 48$

7.2. Non-metric contact and Jacobi structures $\quad 48$

7.3. Further examples of non-metric phase structures

References

\section{InTRODUCTION}

Usually the classical phase space of general relativity is defined to be the subspace of the tangent bundle of spacetime consisting of future oriented timelike vectors. In this phase space we can achieve the standard symplectic formalism for general relativistic analytical mechanics. However, only time normalised vectors have physical meaning, hence one is forced to introduce the corresponding constraint with all associated odd consequences.

On the other hand, there is another possible approach to the phase space, by defining it as the 1st jet space of motions regarded as timelike 1-dimensional submanifolds of spacetime. This viewpoint deals with the essential projective nature of the general relativistic phase space and allows us to skip constraints.

The present paper is aimed at analysing a few basic properties of the phase space according to the 2nd approach. Clearly, the most natural goal is to study the contact and Jacobi structures induced by the Lorentz metric. On the other hand, we could show that the electromagnetic field yields additional terms to the purely metric geometric objects of the phase space. Indeed, the analytical mechanics for a general relativistic particle effected by the electromagnetic fields is to be formulated with these extended geometric objects.

For this reason, in the present paper, we determine the conditions by which the geometric objects induced on the phase space by a general phase connection yield contact and Jacobi structures. Of course, we consider the purely metric framework as particular case. 
Actually, the literature on jets of submanifolds is less popular and more sophisticated than that on jets of fibred manifolds. So, here we sketch a few essential notions on jets of submanifolds in order to make the paper selfconsistent.

We shall be concerned with several geometric structures on the tangent space and on the phase space of spacetime. Some of these structures are well known and some are less standard. Thus, we recall some definitions and introduce new objects, as well. Indeed, the new objects are suitable to capture the non easy objects arising on the phase space.

Throughout the paper, all manifolds and maps will be smooth. If $\boldsymbol{M}$ and $\boldsymbol{N}$ are manifolds, then the sheaf of local maps $\boldsymbol{M} \rightarrow \boldsymbol{N}$ is denoted by $\operatorname{map}(\boldsymbol{M}, \boldsymbol{N}) ;$ if $p: \boldsymbol{F} \rightarrow \boldsymbol{B}$ is a fibred manifold, then the sheaf of local sections $\boldsymbol{B} \rightarrow \boldsymbol{F}$ is denoted by $\sec (\boldsymbol{B}, \boldsymbol{F})$; if $p^{\prime}: \boldsymbol{F}^{\prime} \rightarrow \boldsymbol{B}^{\prime}$ is another fibred manifold, then the sheaf of local fibered maps $\boldsymbol{F} \rightarrow \boldsymbol{F}^{\prime}$ is denoted by fib $\left(\boldsymbol{F}, \boldsymbol{F}^{\prime}\right)$.

Let $M$ be an $m$-dimensional manifold.

If $\Omega$ is a $2-$ form and $\Lambda$ a 2 -vector, then we define the "musical" morphisms

$$
\begin{aligned}
& \Omega^{b}: \sec (\boldsymbol{M}, T \boldsymbol{M}) \rightarrow \sec \left(\boldsymbol{M}, T^{*} \boldsymbol{M}\right): X \mapsto i_{X} \Omega, \\
& \Lambda^{\sharp}: \sec \left(\boldsymbol{M}, T^{*} \boldsymbol{M}\right) \rightarrow \sec (\boldsymbol{M}, T \boldsymbol{M}): \alpha \mapsto i_{\alpha} \Lambda .
\end{aligned}
$$

Let $\boldsymbol{M}$ be a $2 n$-dimensional manifold.

We recall that a symplectic structure [11, pag. 90] is defined by a 2 -form $\Omega$ such that

$$
d \Omega=0, \quad \Omega^{n} \not \equiv 0,
$$

and that a Poisson structure [11, pag. 107] is defined by a 2 -vector $\Lambda$ such that

$$
[\Lambda, \Lambda]=0,
$$

where [,] denotes the Schouten bracket. Moreover, if $\Lambda^{n} \not \equiv 0$, then we say that the Poisson structure is regular.

The musical morphism $\Omega^{b}$ of a symplectic structure turns out to be an isomorphism. If a Poisson structure is regular, then the musical morphism $\Lambda^{\sharp}$ turns out to be an isomorphism. We say that a symplectic structure and a Poisson structure are mutually dual if

$$
\left(\Omega^{b}\right)^{-1}=\Lambda^{\sharp}
$$

Indeed, if $\Omega$ is a symplectic 2 -form and $\Lambda$ the 2 -vector given by $\Lambda^{\sharp}=\left(\Omega^{b}\right)^{-1}$, then $\Lambda$ defines a regular Poisson structure.

Let $\boldsymbol{M}$ be a $(2 n+1)$-dimensional manifold.

We define a covariant pair to be a pair $(\omega, \Omega)$ consisting of a 1 -form $\omega$ and a 2 -form $\Omega$ of constant rank $2 r$, with $0 \leq r \leq n$, such that $\omega \wedge \Omega^{r} \not \equiv 0$, and a contravariant pair to be a pair $(E, \Lambda)$ consisting of a vector field $E$ and a 2 -vector $\Lambda$ of constant rank $2 s$, with $0 \leq s \leq n$, such that $E \wedge \Lambda^{s} \not \equiv 0$. Thus, by definition, we have $\Omega^{r} \not \equiv 0, \Omega^{r+1} \equiv 0$ and $\Lambda^{s} \not \equiv 0, \Lambda^{s+1} \equiv 0$.

We say that the pairs $(\omega, \Omega)$ and $(E, \Lambda)$ are regular if, respectively,

$$
\omega \wedge \Omega^{n} \not \equiv 0 \quad \text { and } \quad E \wedge \Lambda^{n} \not \equiv 0
$$


We recall that a cosymplectic structure [1] is defined by a covariant pair $(\omega, \Omega)$ such that

$$
d \omega=0, \quad d \Omega=0, \quad \omega \wedge \Omega^{n} \not \equiv 0,
$$

and a contact structure [8] is defined by a covariant pair $(\omega, \Omega)$ such that

$$
\Omega=d \omega, \quad \omega \wedge \Omega^{n} \not \equiv 0 .
$$

Equivalently, we can define a contact structure [11, pag. 285] as a 1-form $\omega$ such that

$$
\omega \wedge(d \omega)^{n} \not \equiv 0 .
$$

More generally, we define an almost-cosymplectic-contact structure [8] by a covariant pair $(\omega, \Omega)$ such that

$$
d \Omega=0, \quad \omega \wedge \Omega^{n} \not \equiv 0 .
$$

Thus, an almost-cosymplectic--contact structure becomes a cosymplectic structure when $d \omega=0$ and a contact structure when $\Omega=d \omega$.

We recall that a Jacobi structure [11, pag. 337] is defined by a contravariant pair $(E, \Lambda)$ such that

$$
[E, \Lambda]=0, \quad[\Lambda, \Lambda]=-2 E \wedge \Lambda
$$

In the particular case when $E \equiv 0$, we obtain $[\Lambda, \Lambda]=0$ and the corresponding structure, given by $(E, \Lambda) \equiv(0, \Lambda)$, is a Poisson structure.

Further, a coPoisson structure [8] is defined by a contravariant pair $(E, \Lambda)$ such that

$$
[E, \Lambda]=0, \quad[\Lambda, \Lambda]=0 .
$$

More generally, we define [8] an almost-coPoisson-Jacobi structure by a contravariant pair $(E, \Lambda)$, along with a 1-form $\omega$, called fundamental 1-form, such that

$$
[E, \Lambda]=-E \wedge \Lambda^{\sharp}\left(L_{E} \omega\right), \quad[\Lambda, \Lambda]=2 E \wedge\left(\left(\Lambda^{\sharp} \otimes \Lambda^{\sharp}\right)(d \omega)\right) .
$$

The covariant pair $(\omega, \Omega)$ and the contravariant pair $(E, \Lambda)$ are said to be mutually dual if they are regular, the maps $\Omega^{b}{ }_{\mid \mathrm{im}} \Lambda^{\sharp}: \operatorname{im} \Lambda^{\sharp} \rightarrow \operatorname{im} \Omega^{b}$ and $\Lambda^{\sharp}{ }_{\mid \mathrm{im}} \Omega^{b}: \operatorname{im} \Omega^{b} \rightarrow \operatorname{im} \Lambda^{\sharp}$ are isomorphisms and

$$
\left(\Omega_{\mid \mathrm{im} \Lambda^{\sharp}}\right)^{-1}=\Lambda_{\mid \mathrm{im} \Omega^{b}}^{\sharp}, \quad\left(\Lambda_{\mid \mathrm{im} \Omega^{b}}^{\sharp}\right)^{-1}=\Omega^{b}{ }_{\mid \mathrm{im} \Lambda^{\sharp}}, \quad i_{E} \Omega=0, \quad i_{\omega} \Lambda=0, \quad i_{E} \omega=1 .
$$

1.1. Theorem. [8] Let $(\omega, \Omega)$ and $(E, \Lambda)$ be mutually dual covariant and contravariant pairs. Then,

(1) $(\omega, \Omega)$ is an almost-cosymplectic-contact structure if and only if $(E, \Lambda)$ is an almostcoPoisson-Jacobi structure along with the fundamental 1-form $\omega$;

$(2)(\omega, \Omega)$ is a cosymplectic structure if and only if $(E, \Lambda)$ is a coPoisson structure;

(3) $(\omega, \Omega)$ is a contact structure if and only if $(E, \Lambda)$ is a Jacobi structure.

Actually, the geometric structures arising in this paper, in the framework of the Einstein's phase space, involve mainly the concepts of contact and regular Jacobi structures (eventually, of almost-cosymplectic-contact and regular almost-coPoisson-Jacobi structures). On the other hand, the analogous geometric structures arising in the framework of the Galilei's phase space [7] involve mainly the concepts of cosymplectic and coPoisson structures. 
In order to make our theory explicitly independent from scales, we introduce the "spaces of scales" 9]. Roughly speaking, a space of scales $\mathbb{S}$ has the algebraic structure of $\mathbb{R}^{+}$but has no distinguished 'basis'. We can define the tensor product of spaces of scales and the tensor product of spaces of scales and vector spaces. We can define rational tensor powers $\mathbb{S}^{m / n}$ of a space of scales $\mathbb{S}$. Moreover, we can make a natural identification $\mathbb{S}^{*} \simeq \mathbb{S}^{-1}$.

The basic objects of our theory (the metric field, the phase 2-form, the phase 2-vector, etc.) will be valued into scaled vector bundles, that is into vector bundles multiplied tensorially with spaces of scales. In this way, each tensor field carries explicit information on its "scale dimension".

Actually, we assume the following basic spaces of scales: the space of time intervals $\mathbb{T}$, the space of lengths $\mathbb{L}$ and the space of masses $\mathbb{M}$. We assume the speed of light $c \in \mathbb{T}^{-1} \otimes \mathbb{L}$ and the Planck constant $\hbar \in \mathbb{T}^{-1} \otimes \mathbb{L}^{2} \otimes \mathbb{M}$ as "universal scales". Moreover, we will consider a particle of mass $m \in \mathbb{M}$ and charge $q \in \mathbb{T}^{-1} \otimes \mathbb{L}^{3 / 2} \otimes \mathbb{M}^{1 / 2}$.

\section{Geometry of SPACEtime}

We start by recalling a few basic properties of the Einstein spacetime and of its tangent bundle, [4, 5].

2.1. Spacetime. We assume spacetime to be an oriented 4-dimensional manifold $\boldsymbol{E}$ equipped with a scaled Lorentzian metric $g: \boldsymbol{E} \rightarrow \mathbb{L}^{2} \otimes\left(T^{*} \boldsymbol{E} \otimes T^{*} \boldsymbol{E}\right)$, with signature $(-+++)$; we suppose spacetime to be time oriented. The dual metric will be denoted by

$\bar{g}: \boldsymbol{E} \rightarrow \mathbb{L}^{-2} \otimes(T \boldsymbol{E} \otimes T \boldsymbol{E})$. Actually, our results are essentially valid for any dimension $n \geq 3$ and a pseudo-Riemannian metric of signature $(1, n-1)$.

A spacetime chart is defined to be an ordered chart $\left(x^{0}, x^{i}\right) \in \operatorname{map}\left(\boldsymbol{E}, \mathbb{R} \times \mathbb{R}^{3}\right)$ of $\boldsymbol{E}$, which fits the orientation of spacetime and such that the vector field $\partial_{0}$ is timelike and time oriented and the vector fields $\partial_{1}, \partial_{2}, \partial_{3}$ are spacelike. In the following, we shall always refer to spacetime charts. Greek indices $\lambda, \mu, \ldots$ will span spacetime coordinates, while Latin indices $i, j, \ldots$ will span spacelike coordinates. We have the coordinate expressions

$$
\begin{array}{rlrl}
g & =g_{\lambda \mu} d^{\lambda} \otimes d^{\mu}, & \text { with } & g_{\lambda \mu} \in \operatorname{map}\left(\boldsymbol{E}, \mathbb{L}^{2} \otimes \mathbb{R}\right), \\
\bar{g}=g^{\lambda \mu} \partial_{\lambda} \otimes \partial_{\mu}, & \text { with } & g^{\lambda \mu} \in \operatorname{map}\left(\boldsymbol{E}, \mathbb{L}^{-2} \otimes \mathbb{R}\right) .
\end{array}
$$

The fact that the metric $g$ is a scaled tensor implies that the induced objects of the phase space turn out to be scaled. In order to obtain unscaled objects of the phase space we could replace $g$ with the rescaled Lorentz metric $G=: \frac{m}{\hbar} g: \boldsymbol{E} \rightarrow \mathbb{T} \otimes\left(T^{*} \boldsymbol{E} \otimes T^{*} \boldsymbol{E}\right)$. We leave to the reader the possible task to perform explicitly this easy rescaling throughout the paper.

2.2. Spacetime connections. Let us sketch the notion of general connection of the phase space and related objects. This generality is intended as a preparation for the notion of general connection of the phase space (which is not a vector bundle, hence it does not carry the usual elementary definition of connection) and later in order to allow general theorems concerning geometric structures of the phase space.

We define a spacetime connection to be a connection of the bundle $T \boldsymbol{E} \rightarrow \boldsymbol{E}$.

A spacetime connection can be expressed, equivalently, by a tangent valued form $K: T \boldsymbol{E} \rightarrow T^{*} \boldsymbol{E} \otimes T T \boldsymbol{E}$, which is projectable over 1 $: \boldsymbol{E} \rightarrow T^{*} \boldsymbol{E} \otimes T \boldsymbol{E}$, or by the 
complementary vertical valued form $\nu[K]: T \boldsymbol{E} \rightarrow T^{*} T \boldsymbol{E} \otimes V T \boldsymbol{E}$. Their coordinate expressions are of the type

$$
K=d^{\lambda} \otimes\left(\partial_{\lambda}+K_{\lambda}^{\nu} \dot{\partial}_{\nu}\right), \quad \nu[K]=\left(\dot{d}^{\nu}-K_{\lambda}^{\nu} d^{\lambda}\right) \otimes \dot{\partial}_{\nu}, \quad \text { with } \quad K_{\lambda}^{\nu} \in \operatorname{map}(T \boldsymbol{E}, \mathbb{R}),
$$

where $\left(\partial_{\lambda}, \dot{\partial}_{\lambda}\right)$ and $\left(d^{\lambda}, \dot{d}^{\lambda}\right)$ are the induced bases of local sections of TTE $\rightarrow$ T $\boldsymbol{E}$ and $T^{*} T \boldsymbol{E} \rightarrow T \boldsymbol{E}$, respectively.

Let us consider a spacetime connection $K$.

The connection $K$ is said to be linear if it is a linear fibred morphism over $\mathbf{1}: \boldsymbol{E} \rightarrow$ $T^{*} \boldsymbol{E} \otimes T \boldsymbol{E}$. Thus, the connection $K$ is linear if and only if its coordinate expression is of the type

$$
K_{\lambda}{ }^{\nu}=K_{\lambda}{ }^{\nu}{ }_{\mu} \dot{x}^{\mu}, \quad \text { with } \quad K_{\lambda{ }^{\nu}} \in \operatorname{map}(\boldsymbol{E}, \mathbb{R}) .
$$

The torsion of $K$ is defined to be the vertical valued 2 -form

$$
T \equiv T[K]=: 2[v, K]: T \boldsymbol{E} \rightarrow \Lambda^{2} T^{*} \boldsymbol{E} \otimes V T \boldsymbol{E},
$$

where [,] is the Frölicher-Nijenhuis bracket and $v: T \boldsymbol{E} \rightarrow T^{*} \boldsymbol{E} \otimes V T \boldsymbol{E}$ is the natural vertical valued 1 -form with coordinate expression $v=d^{\lambda} \otimes \dot{\partial}_{\lambda}$. We have the coordinate expression

$$
T=T_{\lambda \mu}{ }^{\nu} d^{\lambda} \wedge d^{\mu} \otimes \dot{\partial}_{\nu}=-2 \dot{\partial}_{\mu} K_{\lambda}^{\nu} d^{\lambda} \wedge d^{\mu} \otimes \dot{\partial}_{\nu}
$$

In the linear case, $T$ can be regarded as the section $T: \boldsymbol{E} \rightarrow \Lambda^{2} T^{*} \boldsymbol{E} \otimes T \boldsymbol{E}$ given, for each vector fields $X$ and $Y$, by $T(X, Y)=\nabla_{X} Y-\nabla_{Y} X-[X, Y]$ and its coordinate expression turns out to be given by the usual formula

$$
T=-\left(K_{\lambda}{ }^{\nu}{ }_{\mu}-K_{\mu}{ }^{\nu}{ }_{\lambda}\right) d^{\lambda} \wedge d^{\mu} \otimes \partial_{\nu} .
$$

Thus, the connection $K$ is linear and torsion free if and only if its coordinate expression is given by $K_{\lambda}{ }^{\nu}=K_{\lambda}{ }^{\nu}{ }_{\mu} \dot{x}^{\mu}$, with $K_{\lambda}{ }^{\nu}{ }_{\mu}=K_{\mu}{ }^{\nu}{ }_{\lambda} \in \operatorname{map}(\boldsymbol{E}, \mathbb{R})$.

The curvature of $K$ is defined to be the vertical valued 2 -form

$$
R \equiv R[K]=:-[K, K]: T \boldsymbol{E} \rightarrow \Lambda^{2} T^{*} \boldsymbol{E} \otimes V T \boldsymbol{E},
$$

where $[$,$] is the Frölicher-Nijenhuis bracket. We have the coordinate expression$

$$
R=R_{\lambda \mu}{ }^{\nu} d^{\lambda} \wedge d^{\mu} \otimes \dot{\partial}_{\nu}=-2\left(\partial_{\lambda} K_{\mu}{ }^{\nu}+K_{\lambda}{ }^{\rho} \dot{\partial}_{\rho} K_{\mu}{ }^{\nu}\right) d^{\lambda} \wedge d^{\mu} \otimes \dot{\partial}_{\nu}
$$

In the linear case, $R$ can be regarded as the section $R: \boldsymbol{E} \rightarrow \Lambda^{2} T^{*} \boldsymbol{E} \otimes T \boldsymbol{E} \otimes T^{*} \boldsymbol{E}$ given, for each vector fields $X, Y$ and $Z$, by $R(X, Y, Z)=\nabla_{X} \nabla_{Y} Z-\nabla_{Y} \nabla_{X} Z-\nabla_{[X, Y]} Z$ and its coordinate expression turns out to be given by the usual formula

$$
R=R_{\lambda \mu}{ }^{\nu}{ }_{\sigma} d^{\lambda} \wedge d^{\mu} \otimes \partial_{\nu} \otimes d^{\sigma}=-2\left(\partial_{\lambda} K_{\mu}{ }^{\nu}{ }+K_{\lambda}{ }^{\rho}{ }_{\sigma} K_{\mu}{ }^{\nu}{ }_{\rho}\right) d^{\lambda} \wedge d^{\mu} \otimes \partial_{\nu} \otimes d^{\sigma} .
$$

We denote by $K[g]$ the Levi-Civita connection, i.e. the torsion free linear spacetime connection such that $\nabla g=0$. We have the usual coordinate expression

$$
K_{\mu}^{\lambda}{ }_{\nu}^{\lambda}=-\frac{1}{2} g^{\lambda \rho}\left(\partial_{\mu} g_{\rho \nu}+\partial_{\nu} g_{\rho \mu}-\partial_{\rho} g_{\mu \nu}\right) \text {. }
$$


2.3. Differential operators. Let us recall some differential operators associated with tangent valued forms and apply them to a spacetime connection, its curvature and the metric.

A spacetime connection $K$ and its curvature $R$, regarded as tangent valued forms of $T \boldsymbol{E}$, yield [10] Lie derivatives of forms of $T \boldsymbol{E}$. Namely, if $\phi \in \sec \left(T \boldsymbol{E}, \Lambda^{r} T^{*} T \boldsymbol{E}\right)$, then we define $L_{K} \phi \in \sec \left(T \boldsymbol{E}, \Lambda^{r+1} T^{*} T \boldsymbol{E}\right)$ and $L_{R} \phi \in \sec \left(T \boldsymbol{E}, \Lambda^{r+2} T^{*} T \boldsymbol{E}\right)$ as

$$
L_{K} \phi=:\left(i_{K} d-d i_{K}\right) \phi \text { and } L_{R} \phi=:\left(i_{R} d+d i_{R}\right) \phi .
$$

In the particular case when $\phi$ is horizontal, i.e. $\phi \in \operatorname{fib}\left(T \boldsymbol{E}, \Lambda^{r} T^{*} \boldsymbol{E}\right)$, we have the coordinate expressions

$$
\begin{aligned}
L_{K} \phi & =\left(\partial_{\lambda_{1}} \phi_{\lambda_{2} \ldots \lambda_{r+1}}+K_{\lambda_{1}}{ }^{\mu} \dot{\partial}_{\mu} \phi_{\lambda_{2} \ldots \lambda_{r+1}}\right) d^{\lambda_{1}} \wedge \ldots \wedge d^{\lambda_{r+1}}, \\
L_{R} \phi & =R_{\lambda_{1} \lambda_{2}}{ }^{\mu} \dot{\partial}_{\mu} \phi_{\lambda_{3} \ldots \lambda_{r+2}} d^{\lambda_{1}} \wedge \ldots \wedge d^{\lambda_{r+2}} .
\end{aligned}
$$

In the further particular case when $K$ (hence also $R$ ) and $\phi$ are linear, the above expressions become

$$
\begin{aligned}
L_{K} \omega & =\left(\partial_{\lambda_{1}} \phi_{\lambda_{2} \ldots \lambda_{r+1} \nu}+K_{\lambda_{1}}{ }^{\mu}{ }_{\nu} \phi_{\lambda_{2} \ldots \lambda_{r+1} \mu}\right) \dot{x}^{\nu} d^{\lambda_{1}} \wedge \ldots \wedge d^{\lambda_{r+1}}, \\
L_{R} \phi & =R_{\lambda_{1} \lambda_{2}}{ }^{\mu}{ }_{\nu} \phi_{\lambda_{3} \ldots \lambda_{r+2} \mu} \dot{x}^{\nu} d^{\lambda_{1}} \wedge \ldots \wedge d^{\lambda_{r+2}} .
\end{aligned}
$$

On the other hand, a linear spacetime connection $K$ yields the covariant exterior differential [10] of cotangent valued forms of $\boldsymbol{E}$. Namely, if $\phi \in \sec \left(\boldsymbol{E}, \Lambda^{r} T^{*} \boldsymbol{E} \otimes T^{*} \boldsymbol{E}\right)$, then we define $d_{K} \phi \in \sec \left(\boldsymbol{E}, \Lambda^{r+1} T^{*} \boldsymbol{E} \otimes T^{*} \boldsymbol{E}\right)$, through the equality

$$
\begin{aligned}
d_{K} \phi\left(X_{1}, \cdots, X_{r+1}\right)(Y)= & \sum_{i=1}^{r+1}(-1)^{i+1} \nabla_{X_{i}}\left(\phi\left(X_{1}, \cdots, \hat{X}_{i}, \cdots, X_{r+1}\right)\right)(Y) \\
& +\sum_{i<j}(-1)^{i+j} \phi\left(\left[X_{i}, X_{j}\right], X_{1}, \ldots, \hat{X}_{i}, \ldots, \hat{X}_{j}, \ldots, X_{r+1}\right)(Y),
\end{aligned}
$$

for each vector fields $X_{1}, \cdots, X_{r+1}, Y$ of $\boldsymbol{E}$, the vector fields $\hat{X}_{i}$ being omitted.

We have the coordinate expression

$$
d_{K} \phi=(r+1)\left(\partial_{\lambda_{1}} \phi_{\lambda_{2} \ldots \lambda_{r+1} \nu}+K_{\lambda_{1}}^{\mu}{ }_{\nu} \phi_{\lambda_{2} \ldots \lambda_{r+1} \mu}\right) d^{\lambda_{1}} \wedge \ldots \wedge d^{\lambda_{r+1}} \otimes d^{\nu} .
$$

Thus, we can compare the Lie derivative and the covariant exterior differential, by considering any cotangent valued $r$-form of $\boldsymbol{E}$ as a linear horizontal $r$-form of $T \boldsymbol{E}$. Indeed, the following result holds.

2.1. Lemma. 4 Let $K$ be a spacetime connection and $\phi$ a linear horizontal $r$-form of $T \boldsymbol{E}$. Then, the Lie derivative $L_{K} \phi$ is a linear horizontal $(r+1)$-form of $T \boldsymbol{E}$ if and only if $K$ is linear. Moreover, in such a case, we have

$$
L_{K} \phi \cong \frac{1}{r+1} d_{K} \phi
$$

2.2. Note. We can apply $d_{K}$ and $L_{K}$ to the scaled metric $g$ as follows.

We can regard $g$ as an $\left(\mathbb{L}^{2} \otimes T^{*} \boldsymbol{E}\right)$-valued 1 -form of $\boldsymbol{E}$. Then, if $K$ is linear, we obtain the covariant exterior differential $d_{K} g$, which is an $\left(\mathbb{L}^{2} \otimes T^{*} \boldsymbol{E}\right)$-valued 2-form given by

$$
\left(d_{K} g\right)(X, Y)(Z)=\left(\nabla_{X}\left(Y^{b}\right)-\nabla_{Y}\left(X^{b}\right)-\left([X, Y]^{b}\right)\right)(Z)
$$




$$
=\left(\nabla_{X} g\right)(Y, Z)-\left(\nabla_{Y} g\right)(X, Z)+g(T(X, Y), Z),
$$

for each vector fields $X, Y, Z$, where $g^{b}: T \boldsymbol{E} \rightarrow \mathbb{L}^{2} \otimes T^{*} \boldsymbol{E}$ denotes the musical map. We have the coordinate expression

$$
\begin{aligned}
& d_{K} g=2\left(\nabla_{\lambda} g_{\mu \rho}-g_{\sigma \rho} K_{\lambda}{ }^{\sigma}{ }_{\mu}\right)\left(d^{\lambda} \wedge d^{\mu}\right) \otimes d^{\rho} \\
& =2\left(\partial_{\lambda} g_{\mu \rho}+g_{\sigma \mu} K_{\lambda}^{\sigma}{ }_{\rho}\right)\left(d^{\lambda} \wedge d^{\mu}\right) \otimes d^{\rho} .
\end{aligned}
$$

On the other hand, we can regard the musical map $g^{b}$ as a scaled linear horizontal 1-form (the metric Liouville 1-form) of T $\boldsymbol{E}$, with coordinate expression $g^{b}=g_{\lambda \mu} \dot{x}^{\lambda} d^{\mu}$. Then, we obtain the Lie derivative $L_{K} g^{b}$, which is a scaled horizontal 2-form of $T \boldsymbol{E}$, with coordinate expression

$$
L_{K} g^{b}=\left(\partial_{\lambda} g_{\rho \mu} \dot{x}^{\rho}+g_{\rho \mu} K_{\lambda}^{\rho}\right) d^{\lambda} \wedge d^{\mu}
$$

hence, if $K$ is linear,

$$
L_{K} g^{b}=\left(\partial_{\lambda} g_{\rho \mu}+g_{\sigma \mu} K_{\lambda}^{\sigma}{ }_{\rho}\right) \dot{x}^{\rho} d^{\lambda} \wedge d^{\mu} .
$$

Thus, if $K$ is linear, we have

$$
L_{K} g^{b} \cong \frac{1}{2} d_{K} g
$$

2.4. Spacetime 2-forms and 2-vectors. Let us analyse the spacetime 2-form and 2 -vector generated by the metric and a spacetime connection.

Let us consider a spacetime connection $K$ and the natural vertical valued 1 -form $v$ : $T \boldsymbol{E} \rightarrow T^{*} \boldsymbol{E} \otimes V T \boldsymbol{E}$, with coordinate expression $v=d^{\lambda} \otimes \dot{\partial}_{\lambda}$.

We define the spacetime 2-form and the spacetime 2-vector of $T \boldsymbol{E}$ associated with $g$ and $K$ to be, respectively, the sections [2, 3, 4]

$$
\begin{aligned}
& \Upsilon=\Upsilon[g, K]=: g\lrcorner(\nu[K] \wedge v): T \boldsymbol{E} \rightarrow \mathbb{L}^{2} \otimes \Lambda^{2} T^{*} T \boldsymbol{E}, \\
& \Xi=\Xi[g, K]=: \bar{g}\lrcorner(K \wedge v) \quad: T \boldsymbol{E} \rightarrow \mathbb{L}^{-2} \otimes \Lambda^{2} T T \boldsymbol{E},
\end{aligned}
$$

with coordinate expressions

$$
\Upsilon=g_{\lambda \mu}\left(\dot{d}^{\lambda}-K_{\nu}{ }^{\lambda} d^{\nu}\right) \wedge d^{\mu} \quad \text { and } \quad \Xi=g^{\lambda \mu}\left(\partial_{\lambda}+K_{\lambda}{ }^{\nu} \dot{\partial}_{\nu}\right) \wedge \dot{\partial}_{\mu},
$$

and, if $K$ is linear,

$$
\Upsilon=g_{\lambda \mu}\left(\dot{d}^{\lambda}-K_{\nu}{ }_{\rho} \dot{x}^{\rho} d^{\nu}\right) \wedge d^{\mu} \quad \text { and } \quad \Xi=g^{\lambda \mu}\left(\partial_{\lambda}+K_{\lambda}{ }_{\rho}{ }_{\rho} \dot{x}^{\rho} \dot{\partial}_{\nu}\right) \wedge \dot{\partial}_{\mu} .
$$

The scaled 2-form and the scaled 2-vector

$$
\begin{aligned}
& \eta=: \Upsilon \wedge \Upsilon \wedge \Upsilon \wedge \Upsilon: T \boldsymbol{E} \rightarrow \mathbb{L}^{8} \otimes \Lambda^{8} T^{*} T \boldsymbol{E} \\
& \bar{\eta}=: \Xi \wedge \Xi \wedge \Xi \wedge \Xi: T \boldsymbol{E} \rightarrow \mathbb{L}^{-8} \otimes \Lambda^{8} T T \boldsymbol{E}
\end{aligned}
$$

turn out to be a scaled volume form and a scaled volume vector, with coordinate expressions

$$
\begin{aligned}
& \eta=4 !|g| \dot{d}^{0} \wedge \dot{d}^{1} \wedge \dot{d}^{2} \wedge \dot{d}^{3} \wedge d^{0} \wedge d^{1} \wedge d^{2} \wedge d^{3} \\
& \bar{\eta}=4 !|\bar{g}| \dot{\partial}_{0} \wedge \dot{\partial}_{1} \wedge \dot{\partial}_{2} \wedge \dot{\partial}_{3} \wedge \partial_{0} \wedge \partial_{1} \wedge \partial_{2} \wedge \partial_{3} .
\end{aligned}
$$

We have [4] $i_{\Xi} \Upsilon=-4$ and $\Xi^{\sharp}=\left(\Upsilon^{b}\right)^{-1}$; thus, $\Upsilon$ and $\Xi$ are mutually dual. Here, we naturally identify $\Upsilon^{b}: T T \boldsymbol{E} \rightarrow \mathbb{L}^{2} \otimes T^{*} T \boldsymbol{E}$ with $\Upsilon^{b}: \mathbb{L}^{-2} \otimes T T \boldsymbol{E} \rightarrow T^{*} T \boldsymbol{E}$. 
In view of forthcoming considerations, let us consider a linear spacetime connection $K$, the scaled tangent space $\tilde{T} \boldsymbol{E}=: \mathbb{T}^{*} \otimes T \boldsymbol{E}$ and the natural vertical valued 1 -form $\tilde{v}: \tilde{T} \boldsymbol{E} \rightarrow T^{*} \tilde{T} \boldsymbol{E} \otimes V \tilde{T} \boldsymbol{E}$, with coordinate expression $\tilde{v}=d_{0}^{\lambda} \otimes \dot{\partial}_{\lambda}^{0}$.

We observe that $K$ induces naturally a linear connection $\tilde{K}$ on the tensor product bundle $\mathbb{T}^{*} \otimes T \boldsymbol{E}$, via the tensor product of the natural flat linear connection of the trivial bundle $\boldsymbol{E} \times \mathbb{T}^{*} \rightarrow \boldsymbol{E}$ and the linear connection $K$. Indeed, the connection $\tilde{K}$ can be regarded as a tangent valued form $\tilde{K}: \tilde{T} \boldsymbol{E} \rightarrow T^{*} \boldsymbol{E} \otimes T \tilde{T} \boldsymbol{E}$, or as the complementary vertical valued form $\nu[\tilde{K}]: T \tilde{T} \boldsymbol{E} \rightarrow V \tilde{T} \boldsymbol{E}$, with coordinate expressions

$$
\tilde{K}=d^{\lambda} \otimes\left(\partial_{\lambda}+K_{\lambda}{ }^{\mu}{ }_{\nu} \dot{x}_{0}^{\nu} \dot{\partial}_{\mu}^{0}\right) \quad \text { and } \quad \nu[\tilde{K}]=\left(\dot{d}_{0}^{\mu}-K_{\lambda}{ }^{\mu}{ }_{\nu} \dot{x}_{0}^{\nu} d^{\lambda}\right) \otimes \dot{\partial}_{\mu}^{0} .
$$

We can refrase the previous construction by replacing, respectively, $T \boldsymbol{E}, K$ and $v$ with $\tilde{T} \boldsymbol{E}, \tilde{K}$ and $\tilde{v}$.

We define the scaled spacetime 2-form and the scaled spacetime 2-vector of $\tilde{T} \boldsymbol{E}$, associated with $g$ and $\tilde{K}$, to be the scaled sections

$$
\begin{aligned}
& \widetilde{\Upsilon}=\widetilde{\Upsilon}[g, \tilde{K}]=: g\lrcorner(\nu[\tilde{K}] \wedge \tilde{v}): \tilde{T} \boldsymbol{E} \rightarrow\left(\mathbb{T}^{*} \otimes \mathbb{L}^{2}\right) \otimes \Lambda^{2} T^{*} \tilde{T} \boldsymbol{E}, \\
& \widetilde{\Xi}=\widetilde{\Xi}[g, \tilde{K}]=: \bar{g}\lrcorner(\tilde{K} \wedge \tilde{v}) \quad: \tilde{T} \boldsymbol{E} \rightarrow\left(\mathbb{T} \otimes \mathbb{L}^{-2}\right) \otimes \Lambda^{2} T \tilde{T} \boldsymbol{E},
\end{aligned}
$$

with coordinate expressions

$$
\widetilde{\Upsilon}=g_{\lambda \mu} u^{0} \otimes\left(\dot{d}_{0}^{\lambda}-K_{\nu}{ }_{\rho}{ }_{\rho} \dot{x}_{0}^{\rho} d^{\nu}\right) \wedge d^{\mu} \quad \text { and } \quad \widetilde{\Xi}=g^{\lambda \mu} u_{0} \otimes\left(\partial_{\lambda}+K_{\lambda}{ }_{\rho}{ }_{\rho} \dot{x}_{0}^{\rho} \dot{\partial}_{\nu}^{0}\right) \wedge \dot{\partial}_{\mu}^{0} \text {. }
$$

We have [4] $i_{\widetilde{\Xi}} \widetilde{\Upsilon}=-4$ and $\widetilde{\Xi}^{\sharp}=\left(\widetilde{\Upsilon}^{b}\right)^{-1}$; thus, $\widetilde{\Upsilon}$ and $\widetilde{\Xi}$ are mutually dual. Here, we naturally identify $\widetilde{\Upsilon}^{b}: T \tilde{T} \boldsymbol{E} \rightarrow \mathbb{T}^{*} \otimes \mathbb{L}^{2} \otimes T^{*} \tilde{T} \boldsymbol{E}$ with $\tilde{\Upsilon}^{b}: \mathbb{T} \otimes \mathbb{L}^{-2} \otimes T \tilde{T} \boldsymbol{E} \rightarrow T^{*} \tilde{T} \boldsymbol{E}$.

\section{Geometric structures of the tangent Bundle}

Next, we recall a few results [4] on symplectic and Poisson structures induced on the tangent bundle of spacetime by the metric $g$ and a spacetime connection $K$, and add some new results as well.

3.1. General spacetime connection case. Let us consider a spacetime connection $K$, its curvature $R=R[K]$, the spacetime 2-form $\Upsilon=\Upsilon[g, K]$ and the spacetime 2-vector $\Xi=\Xi[g, K]$. Let $I=\dot{x}^{\lambda} \dot{\partial}_{\lambda}$ be the Liouville vector field of $T \boldsymbol{E}$.

3.1. Theorem. [4] The following conditions are equivalent:
(1) $L_{I} L_{K} g^{b}=0$ and $L_{R} g^{b}=0$.
(2) $d \Upsilon=0$.
(3) $[\Xi, \Xi]=0$

3.2. Corollary. $\Upsilon$ is a scaled symplectic 2 -form and $\Xi$ a scaled Poisson 2 -vector if and only if $L_{I} L_{K} g^{b}=0$ and $L_{R} g^{b}=0$.

3.3. Corollary. The condition $L_{K} g^{b}=0$ implies $L_{I} L_{K} g^{b}=0$ and $L_{R} g^{b}=0$, hence it implies that $\Upsilon$ is a scaled symplectic 2 -form and $\Xi$ a scaled Poisson 2 -vector.

3.4. Lemma. The 2 -form $\Upsilon+L_{K} g^{b}$ is exact and more precisely we have

$$
\Upsilon+L_{K} g^{b}=d g^{b} .
$$


Hence, we have

$$
d \Upsilon=-d L_{K} g^{b}
$$

Proof. We have

$$
\begin{aligned}
\Upsilon+L_{K} g^{b} & =g_{\lambda \mu}\left(\dot{d}^{\lambda}-K_{\nu}{ }^{\lambda} d^{\nu}\right) \wedge d^{\mu}+\left(\partial_{\lambda} g_{\rho \mu} \dot{x}^{\rho}+g_{\rho \mu} K_{\lambda}{ }^{\rho}\right) d^{\lambda} \wedge d^{\mu} \\
& =\dot{\partial}_{\lambda}\left(g_{\rho \mu} \dot{x}^{\rho}\right) \dot{d}^{\lambda} \wedge d^{\mu}+\partial_{\lambda}\left(g_{\rho \mu} \dot{x}^{\rho}\right) d^{\lambda} \wedge d^{\mu} \\
& =d\left(g_{\rho \mu} \dot{x}^{\rho} d^{\mu}\right)=d g^{b}
\end{aligned}
$$

3.5. Theorem. The following conditions are equivalent:
(1) $L_{K} g^{b}=0$;
(2) $\Upsilon=d g^{b}$.

3.2. Linear spacetime connection case. Let us consider a linear spacetime connection $K$ and the induced scaled spacetime 2 -form $\Upsilon=\Upsilon[g, K]$ and scaled spacetime 2 -vector $\Xi=\Xi[g, K]$.

3.6. Theorem. 4] The following equalities are equivalent:
(1) $L_{K} g^{b}=0$;
(2) $d_{K} g=0$;
(3) $\Upsilon=d g^{b}$;
(4) $[\Xi, \Xi]=0$.

3.7. Corollary. $\Upsilon$ is a scaled symplectic 2 -form and $\Xi$ a scaled Poisson 2 -vector if and only if $d_{K} g=0=L_{K} g^{b}$.

3.8. Note. If $K$ is torsion free, then $\nabla g$ is symmetric if and only if $d_{K} g=0=L_{K} g^{b}$.

In fact, in virtue of Note 2.2 , we have $d_{K} g=0=L_{K} g^{b}$ if and only if $\left(\nabla_{X} g\right)(Y, Z)=$ $\left(\nabla_{Y} g\right)(X, Z)$.

3.9. Theorem. [4 If $K$ is torsion free, then the following conditions are equivalent:
(1) $\nabla g$ is symmetric;
(2) $d \Upsilon=0$;
(3) $[\Xi, \Xi]=0$.

3.10. Corollary. Let $K$ be torsion free. Then, $\Upsilon$ is a scaled symplectic 2 -form and $\Xi$ a scaled Poisson $2-$ vector if and only if $\nabla g$ is symmetric.

3.11. Corollary. If $K=K[g]$ is the Levi-Civita connection, then we obtain the metric scaled symplectic 2-form and scaled Poisson 2-vector

$$
\Upsilon=\Upsilon[g]=: \Upsilon[g, K[g]] \quad \text { and } \quad \Xi=\Xi[g]=: \Xi[g, K[g]] \text {, }
$$

respectively. In this case, we have $\Upsilon=d g^{b}$.

3.3. Non-metric spacetime connection case. We have seen that the metric $g$ yields naturally the metric scaled symplectic 2 -form $\Upsilon=\Upsilon[g]$ and the metric scaled Poisson 2 -vector $\Xi=\Xi[g]$. Now, we discuss the case of "non-metric" connections $K$ yielding a scaled spacetime 2 -form $\Upsilon=: \Upsilon[g, K]$, which fulfills the equality $\Upsilon=d g^{b}$.

3.12. Note. The equality

$$
K=K[g]+\Phi
$$

yields a bijection between spacetime connections $K$ and sections $\Phi: T \boldsymbol{E} \rightarrow T^{*} \boldsymbol{E} \otimes V T \boldsymbol{E}$, with coordinate expression $\Phi=\Phi_{\lambda}{ }^{\mu} d^{\lambda} \otimes \dot{\partial}_{\mu}$, where $\Phi_{\lambda}^{\mu} \in \operatorname{map}(T \boldsymbol{E}, \mathbb{R})$. 
On the other hand, by identifying $V T \boldsymbol{E}$ with $T \boldsymbol{E} \times{ }_{\boldsymbol{E}} T \boldsymbol{E}$, the equalities

$$
\Phi=: \operatorname{proj}_{2} \circ \Phi, \quad \Phi=v(\underline{\Phi}) \quad \text { and } \quad \phi=: g^{b}(\underline{\Phi}), \quad \Phi=g^{\sharp 2}(\phi)
$$

yield bijections between the sections $\Phi: T \boldsymbol{E} \rightarrow T^{*} \boldsymbol{E} \otimes V T \boldsymbol{E}$ and the fibred morphisms $\Phi: T \boldsymbol{E} \rightarrow T^{*} \boldsymbol{E} \otimes T \boldsymbol{E}$ and $\phi: T \boldsymbol{E} \rightarrow \mathbb{L}^{2} \otimes\left(T^{*} \boldsymbol{E} \otimes T^{*} \boldsymbol{E}\right)$.

Let us consider a spacetime connection $K=K[g]+\Phi=K[g]+v(\Phi)$ and the induced scaled spacetime 2 -form $\Upsilon=\Upsilon[g, K]$.

3.13. Theorem. The following conditions are equivalent:
(1) $\Upsilon=d g^{b}$;
(2) $L_{\Phi} g^{b}=0$;
(3) $\phi$ is symmetric.

Proof. In virtue of Theorem [3.6, $\Upsilon=d g^{b}$ if and only if $L_{K} g^{b}=0$, i.e. if and only if $L_{\Phi} g^{b}=0$, i.e., in coordinates, if and only if $g_{\lambda \rho} \Phi_{\mu}{ }^{\rho} d^{\lambda} \wedge d^{\mu}=0$. Hence, $L_{\Phi} g^{b}=0$ if and only if $\phi$ is symmetric.

3.14. Example. The connection $K=K[g]+v$ is a "non-metric" non-linear spacetime connection yielding the scaled symplectic 2 -form $\Upsilon=d g^{b}$.

3.15. Note. It is easy to see that $K$ is linear if and only if $\Phi$ is linear, i.e. if and only if $\phi$ can be identified with a section $\phi: \boldsymbol{E} \rightarrow \mathbb{L}^{2} \otimes T^{*} \boldsymbol{E} \otimes T^{*} \boldsymbol{E} \otimes T^{*} \boldsymbol{E}$ which is symmetric in the 1st two factors.

Moreover, if $\phi$ is symmetric in all factors, then $K$ turns out to be torsion free.

3.16. Note. We recall [14] that a linear torsion free connection $K$ is projectively equivalent to the metric connection $K[g]$ if and only if $K$ and $K[g]$ have the same unparametrized geodesics, i.e., in coordinates, if and only if

$$
K_{\lambda}{ }^{\nu}{ }_{\mu}=K[g]_{\lambda}{ }^{\nu}{ }_{\mu}+\delta_{\lambda}^{\nu} \psi_{\mu}+\delta_{\mu}^{\nu} \psi_{\lambda}, \quad \text { where } \quad \psi_{\lambda} \in \operatorname{map}(\boldsymbol{E}, \mathbb{R}) .
$$

It is easy to see that any torsion free linear spacetime connection $K$ projectively equivalent to $K[g]$ is such that $\Upsilon=d g^{b}$ and it is associated with a symmetric $(0,3)$-tensor field of the type $\phi=g \odot \psi$, for a certain spacetime 1-form $\psi$.

But not all torsion free linear spacetime connections $K$ such that $\Upsilon=d g^{b}$ are projectively equivalent to $K[g]$. Hence, the condition $d_{K} g=0$ is more general than the condition for connections projectively equivalent to $K[g]$.

3.17. Note. Let $K$ and $K^{\prime}$ be two spacetime connections and consider the difference tensor $\Phi=: K-K^{\prime}: T \boldsymbol{E} \rightarrow T^{*} \boldsymbol{E} \otimes V T \boldsymbol{E}$. Then, the following conditions are equivalent:

1) $\Upsilon[g, K]=\Upsilon\left[g, K^{\prime}\right]$

2) $\Xi[g, K]=\Xi\left[g, K^{\prime}\right]$,

3) $\phi=: g^{b}(\underline{\Phi})$ is symmetric.

Thus, the relation $\Upsilon[g, K]=\Upsilon\left[g, K^{\prime}\right]$, or equivalently, the relation $\Xi[g, K]=\Xi\left[g, K^{\prime}\right]$, defines an equivalence relation on the space of spacetime connections.

We are mainly interested in the spacetime connections which are equivalent to $K[g]$, because they yield an exact spacetime 2 -form $\Upsilon$. 


\section{Geometry of the Einstein phase space}

Next, we study the geometric properties of the phase space of a classical particle in the Lorentzian framework, by adding several new results with respect to [5].

4.1. Jets of submanifolds. In view of the definition of the phase space, let us consider a manifold $\boldsymbol{M}$ of dimension $n$ and recall a few basic facts concerning jets of submanifolds.

Let $k \geq 0$ be an integer. A $k$-jet of 1 -dimensional submanifolds of $\boldsymbol{M}$ at $x \in \boldsymbol{M}$ is defined to be an equivalence class of 1-dimensional submanifolds touching each other at $x$ with a contact of order $k$. The $k$-jet of a 1-dimensional submanifold $s: \boldsymbol{N} \subset \boldsymbol{M}$ at $x \in \boldsymbol{N}$ is denoted by $j_{k} s(x)$. The set of all $k$-jets of all 1 -dimensional submanifolds at $x \in \boldsymbol{M}$ is denoted by $J_{k x}(\boldsymbol{M}, 1)$. The set $J_{k}(\boldsymbol{M}, 1)=: \bigsqcup_{x \in M} J_{k x}(\boldsymbol{M}, 1)$ is said to be the $k$-jet space of 1 -dimensional submanifolds of $\boldsymbol{M}$. In particular, for $k=0$, we have the natural identification $J_{0}(\boldsymbol{M}, 1)=\boldsymbol{M}$, given by $j_{0} s(x)=x$, for each 1 -dimensional submanifold $s: \boldsymbol{N} \subset \boldsymbol{M}$. For each integers $k \geq h \geq 0$, we have the natural projection $\pi_{h}^{k}: J_{k}(\boldsymbol{M}, 1) \rightarrow J_{h}(\boldsymbol{M}, 1): j_{k} s(x) \mapsto j_{h} s(x)$.

For each 1-dimensional submanifold $s: \boldsymbol{N} \subset \boldsymbol{M}$ and each integer $k \geq 0$, we have the $\operatorname{map} j_{k} s: \boldsymbol{N} \rightarrow J_{k}(\boldsymbol{M}, 1): x \mapsto j_{k} s(x)$.

A chart of $\boldsymbol{M}$ is said to be divided if the set of its coordinate functions is divided into two subsets of 1 and $n-1$ elements. Our typical notation for a divided chart will be $\left(x^{0}, x^{i}\right)$, with $1 \leq i \leq n-1$. A divided chart and a 1-dimensional submanifold $s: \boldsymbol{N} \subset \boldsymbol{M}$ are said to be related if the map $\breve{x}^{0}=:\left.x^{0}\right|_{\boldsymbol{N}} \in \operatorname{map}(\boldsymbol{N}, \mathbb{R})$ is a chart of $\boldsymbol{N}$. In such a case, the submanifold $\boldsymbol{N}$ is locally characterised by $s^{i} \circ\left(\breve{x}^{0}\right)^{-1}=:\left(x^{i} \circ s\right) \circ\left(\breve{x}^{0}\right)^{-1} \in \operatorname{map}(\mathbb{R}, \mathbb{R})$. In particular, if the divided chart is adapted to the submanifold, then the chart and the submanifold are related.

Let us consider a divided chart $\left(x^{0}, x^{i}\right)$ of $\boldsymbol{M}$.

Then, for each submanifold $s: \boldsymbol{N} \subset \boldsymbol{M}$ which is related to this chart, the chart yields naturally the local fibred chart $\left(x^{0}, x^{i} ; x_{\alpha}^{i}\right)_{1 \leq|\underline{\alpha}| \leq k} \in \operatorname{map}\left(J_{k}(\boldsymbol{M}, 1), \mathbb{R}^{n} \times \mathbb{R}^{k(n-1)}\right)$ of $J_{k}(\boldsymbol{M}, 1)$, where $\underline{\alpha}=:(h)$ is a multi-index, of "range" 1 and "length" $|\underline{\alpha}|=h$, and the functions $x_{\underline{\alpha}}^{i}$ are defined by $x_{\underline{\alpha}}^{i} \circ j_{1} \boldsymbol{N}=: \partial_{0 \ldots 0} s^{i}$, with $1 \leq|\underline{\alpha}| \leq k$.

We can prove the following facts:

$1)$ the above charts $\left(x^{0}, x^{i} ; x_{\alpha}^{i}\right)$ yield a smooth structure of $J_{k}(\boldsymbol{M}, 1)$;

2) for each 1-dimensional submanifold $s: \boldsymbol{N} \subset \boldsymbol{M}$ and for each integer $k \geq 0$, the subset $j_{k} s(\boldsymbol{N}) \subset J_{k}(\boldsymbol{M}, 1)$ turns out to be a smooth 1-dimensional submanifold;

3) for each integers $k \geq h \geq 1$, the maps $\pi_{h}^{k}: J_{k}(\boldsymbol{M}, 1) \rightarrow J_{h}(\boldsymbol{M}, 1)$ turn out to be smooth bundles.

We shall always refer to such diveded charts $\left(x^{0}, x^{i}\right)$ of $\boldsymbol{M}$ and to the induced fibred charts $\left(x^{0}, x^{i} ; x_{\alpha}^{i}\right)$ of $J_{k}(\boldsymbol{M}, 1)$.

Let $m_{1} \in J_{1}(\overline{\boldsymbol{M}}, 1)$, with $m_{0}=\pi_{0}^{1}\left(m_{1}\right) \in \boldsymbol{M}$. Then, the tangent spaces at $m_{0}$ of all $1-$ dimensional submanifolds $\boldsymbol{N}$, such that $j_{1} s\left(m_{0}\right)=m_{1}$, coincide. Accordingly, we denote by $T\left[m_{1}\right] \subset T_{m_{0}} \boldsymbol{M}$ the tangent space at $m_{0}$ of the above 1-dimensional submanifolds $\boldsymbol{N}$ generating $m_{1}$. We have the natural fibred isomorphism $J_{1}(\boldsymbol{M}, 1) \rightarrow \operatorname{Grass}(\boldsymbol{M}, 1)$ : $m_{1} \mapsto T\left[m_{1}\right] \subset T_{m_{0}} \boldsymbol{M}$ over $\boldsymbol{M}$ of the 1st jet bundle with the Grassmannian bundle of dimension 1. If $s: \boldsymbol{N} \subset \boldsymbol{M}$ is a submanifold, then we obtain $T\left[j_{1} s\right]=\operatorname{span}\left\langle\partial_{0}+\partial_{0} s^{i} \partial_{i}\right\rangle$, with reference to a related chart. 
4.2. Phase space. Let us introduce the phase space of a classical particle and its basic contact structure induced by the Lorentz metric.

A motion is defined to be a 1-dimensional timelike submanifold $s: \boldsymbol{T} \subset \boldsymbol{E}$. Let us consider a motion $s: \boldsymbol{T} \subset \boldsymbol{E}$.

For every arbitrary choice of a "proper time origin" $t_{0} \in \boldsymbol{T}$, we obtain the "proper time scaled function" given by the equality $\sigma: T \rightarrow \overline{\mathbb{T}}: t \mapsto \frac{1}{c} \int_{\left[t_{0}, t\right]}\left\|\frac{d s}{d \breve{x}^{0}}\right\| d \breve{x}^{0}$.

This map yields, at least locally, a bijection $\boldsymbol{T} \rightarrow \overline{\mathbb{T}}$, hence a (local) affine structure of $\boldsymbol{T}$ associated with the vector space $\overline{\mathbb{T}}$. Indeed, this (local) affine structure does not depend on the choice of the proper time origin and of the spacetime chart.

Let us choose a time origin $t_{0} \in \boldsymbol{T}$ and consider the associated proper time scaled function $\sigma: \boldsymbol{T} \rightarrow \overline{\mathbb{T}}$ and the induced linear isomorphism $T \boldsymbol{T} \rightarrow \boldsymbol{T} \times \overline{\mathbb{T}}$. Moreover, let us consider a spacetime chart $\left(x^{\lambda}\right)$ and the induced chart $\left(\breve{x}^{0}\right) \in \operatorname{map}(\boldsymbol{T}, \mathbb{R})$. Let us set $\partial_{0} s^{\lambda}=: \frac{d s^{\lambda}}{d \breve{x}^{0}}$.

The 1 st differential of the motion $s$ is the map $d s=: \frac{d s}{d \sigma}: \boldsymbol{T} \rightarrow \mathbb{T}^{*} \otimes T \boldsymbol{E}$.

We have $g(d s, d s)=-c^{2}$ and the coordinate expression

$$
d s=\frac{d s^{\lambda}}{d \sigma}\left(\partial_{\lambda} \circ s\right)=\frac{c_{0} u^{0} \otimes\left(\left(\partial_{0} \circ s\right)+\partial_{0} s^{i}\left(\partial_{i} \circ s\right)\right)}{\sqrt{\left|\left(g_{00} \circ s\right)+2\left(g_{0 j} \circ s\right) \partial_{0} s^{j}+\left(g_{i j} \circ s\right) \partial_{0} s^{i} \partial_{0} s^{j}\right|}} .
$$

We define the phase space of a classical particle to be the subspace $\mathcal{J}_{1} \boldsymbol{E} \subset J_{1}(\boldsymbol{E}, 1)$ consisting of all 1 -jets of motions.

For each 1-dimensional submanifold $s: \boldsymbol{T} \subset \boldsymbol{E}$ and for each $x \in \boldsymbol{T}$, we have $j_{1} s(x) \in$ $\mathcal{\partial}_{1} \boldsymbol{E}$ if and only if $T\left[j_{1} s(x)\right]=T_{x} \boldsymbol{T}$ is timelike.

Any spacetime chart $\left(x^{0}, x^{i}\right)$ is related to each motion $s: \boldsymbol{T} \rightarrow \boldsymbol{E}$. Hence, the fibred chart $\left(x^{0}, x^{i}, x_{0}^{i}\right)$ is defined on tubelike open subsets of $\partial_{1} \boldsymbol{E}$.

We shall always refer to the above fibred charts.

The velocity of a motion $s: \boldsymbol{T} \subset \boldsymbol{E}$ is defined to be its 1-jet $j_{1} s: \boldsymbol{T} \rightarrow \mathcal{J}_{1}(\boldsymbol{E}, 1)$.

We define the contact map to be the unique fibred morphism д : $\mathcal{J}_{1} \boldsymbol{E} \rightarrow \mathbb{T}^{*} \otimes$ Т $\boldsymbol{E}$ over $\boldsymbol{E}$, such that д о $j_{1} s=d s$, for each motion $s: \boldsymbol{T} \rightarrow T \boldsymbol{E}$. We have the coordinate expression д $=c \alpha^{0}\left(\partial_{0}+x_{0}^{i} \partial_{i}\right)$, where we have set $\alpha^{0}=: 1 / \sqrt{\left|g_{00}+2 g_{0 j} x_{0}^{j}+g_{i j} x_{0}^{i} x_{0}^{j}\right|}$.

We have $g\left(\right.$ д, д) $=-c^{2}$.

The fibred morphism д : $\mathcal{\partial}_{1} \boldsymbol{E} \rightarrow \mathbb{T}^{*} \otimes T \boldsymbol{E}$ is injective. Indeed, it makes $\mathcal{J}_{1} \boldsymbol{E} \subset \mathbb{T}^{*} \otimes T \boldsymbol{E}$ the fibred submanifold over $\boldsymbol{E}$ characterised by the constraint $g_{\lambda \mu} \dot{x}_{0}^{\lambda} \dot{x}_{0}^{\mu}=-\left(c_{0}\right)^{2}$.

We define the time form to be the 1 -jet based scaled 1 -form $\tau=:-\frac{1}{c^{2}} g^{b}\left(\right.$ д) $: \mathcal{J}_{1} \boldsymbol{E} \rightarrow$ $\mathbb{T} \otimes T^{*} \boldsymbol{E}$, with coordinate expression $\tau=\tau_{\lambda} d^{\lambda}$, where $\tau_{\lambda}=-\frac{\alpha^{0}}{c}\left(g_{0 \lambda}+g_{i \lambda} x_{0}^{i}\right)$.

We have $\tau($ д $)=1$ and $\bar{g}(\tau, \tau)=-\frac{1}{c^{2}}$.

We define the complementary contact map to be the linear fibred morphism $\theta=: 1-$ д $\otimes \tau: \mathcal{\partial}_{1} \boldsymbol{E} \underset{\boldsymbol{E}}{\boldsymbol{E}}$ T $\boldsymbol{E} \rightarrow T \boldsymbol{E}$, over $\mathcal{\partial}_{1} \boldsymbol{E}$, given by $\theta(v)=v-\tau(v)$ д. We have the coordinate expressions $\theta=d^{\lambda} \otimes \partial_{\lambda}+\left(\alpha^{0}\right)^{2}\left(g_{0 \lambda}+g_{i \lambda} x_{0}^{i}\right) d^{\lambda} \otimes\left(\partial_{0}+x_{0}^{j} \partial_{j}\right)$.

4.3. Orthogonal splittings. We have a natural orthogonal splitting of the tangent and cotangent spaces of spacetime pullbacked over the phase space.

We define

- the д-horizontal tangent space of spacetime, 
- the $\tau$-vertical tangent space of spacetime,

- the $\tau$-horizontal cotangent space of spacetime,

- the д-vertical cotangent space of spacetime

to be, respectively, the vector subbundles over $\mathcal{J}_{1} \boldsymbol{E}$

$$
\begin{aligned}
& H_{\text {д }} \boldsymbol{E}=:\left\{\left(e_{1}, X\right) \in \underset{\mathcal{J}_{1}}{\boldsymbol{E}} \underset{\boldsymbol{E}}{\times} T \boldsymbol{E} \mid X \in T\left[e_{1}\right]\right\} \quad \subset \mathcal{J}_{1} \underset{\boldsymbol{E}}{\times} \underset{\boldsymbol{E}}{\times} T \boldsymbol{E}, \\
& V_{\tau} \boldsymbol{E}=:\left\{\left(e_{1}, X\right) \in \underset{\mathcal{J}_{1}}{\boldsymbol{E}} \underset{\boldsymbol{E}}{\times} T \boldsymbol{E} \mid X \in T\left[e_{1}\right]^{\perp}\right\} \quad \subset \mathcal{J}_{1} \boldsymbol{E} \underset{\boldsymbol{E}}{\times} T \boldsymbol{E},
\end{aligned}
$$

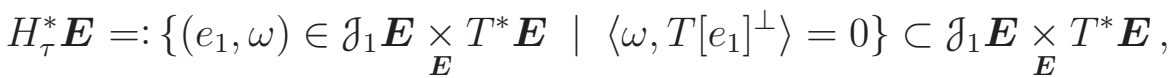

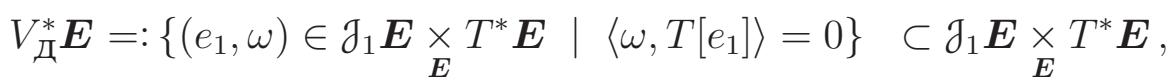

where $T\left[e_{1}\right]$ is the tangent space associated with the 1 st jet $e_{1}$ and $T\left[e_{1}\right]^{\perp}$ is its orthogonal.

Indeed, $H_{\text {д }} \boldsymbol{E}$ and $H_{\tau}^{*} \boldsymbol{E}$ are generated by д and $\tau$, respectively; moreover, $V_{\tau} \boldsymbol{E}$ and $V_{\text {Д }}^{*} \boldsymbol{E}$ are generated by $\theta$ and $\theta^{*}$, respectively.

4.1. Proposition. We have the natural orthogonal linear fibred splittings over $\mathcal{J}_{1} \boldsymbol{E}$

$$
\partial_{1} \boldsymbol{E} \underset{\boldsymbol{E}}{\times} T \boldsymbol{E}=H_{\mathrm{Z}} \boldsymbol{E} \underset{\partial_{1} \boldsymbol{E}}{\oplus} V_{\tau} \boldsymbol{E} \quad \text { and } \quad \partial_{1} \boldsymbol{E} \underset{\boldsymbol{E}}{\times} T^{*} \boldsymbol{E}=H_{\tau}^{*} \boldsymbol{E} \underset{\partial_{1} \boldsymbol{E}}{\oplus} V_{\text {Д }}^{*} \boldsymbol{E}
$$

and the corresponding projections

$$
\begin{aligned}
& \pi^{\|}=\tau \otimes \text { д : } \mathcal{\partial}_{1} \underset{\boldsymbol{E}}{\boldsymbol{E}} \underset{\boldsymbol{E}}{\mathrm{E}} \boldsymbol{\boldsymbol { E }} \rightarrow H_{\text {Д }} \boldsymbol{E} \quad \text { and } \quad \pi_{\|}=\text {д } \otimes \tau: \mathcal{\partial}_{1} \underset{\boldsymbol{E}}{\boldsymbol{E}} T^{*} \boldsymbol{E} \rightarrow H_{\tau}^{*} \boldsymbol{E},
\end{aligned}
$$

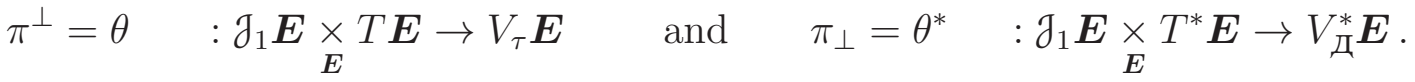

We have a natural identification $\left(H_{\text {Д }} \boldsymbol{E}\right)^{*}=H_{\tau}^{*} \boldsymbol{E}$ and $\left(V_{\tau} \boldsymbol{E}\right)^{*}=V_{\text {Д }}^{*} \boldsymbol{E}$.

4.2. Lemma. The restrictions of $g$ and $\bar{g}$ to the components of the above splitting are

$$
\begin{array}{lll}
g_{\|}=: g \circ\left(\pi^{\|}, \pi^{\|}\right)=-c^{2} \tau \otimes \tau & \text { and } & \bar{g}^{\|}=: \bar{g} \circ\left(\pi_{\|}, \pi_{\|}\right)=-\frac{1}{c^{2}} \text { д } \otimes \text { д , } \\
g_{\perp}=: g \circ\left(\pi^{\perp}, \pi^{\perp}\right)=g+c^{2} \tau \otimes \tau & \text { and } & \bar{g}^{\perp}=: \bar{g} \circ\left(\pi_{\perp}, \pi_{\perp}\right)=\bar{g}+\frac{1}{c^{2}} \text { д } \otimes \text { д . }
\end{array}
$$

We have the mutually dual local bases $\left(b_{0}, b_{i}\right)$ and $\left(\beta^{0}, \beta^{i}\right)$ adapted to the above splittings, where

$$
\begin{aligned}
b_{0}=: \partial_{0}+x_{0}^{i} \partial_{i} & \in \operatorname{fib}\left(\partial_{1} \boldsymbol{E}, H_{\text {Д }} \boldsymbol{E}\right), \\
b_{i}=: \partial_{i}-c \alpha^{0} \tau_{i}\left(\partial_{0}+x_{0}^{j} \partial_{j}\right) & \in \operatorname{fib}\left(\mathcal{\partial}_{1} \boldsymbol{E}, V_{\tau} \boldsymbol{E}\right), \\
\beta^{0}=: d^{0}+c \alpha^{0} \tau_{i}\left(d^{i}-x_{0}^{i} d^{0}\right) & \in \operatorname{fib}\left(\mathcal{\partial}_{1} \boldsymbol{E}, H_{\tau}^{*} \boldsymbol{E}\right), \\
\beta^{i}=: d^{i}-x_{0}^{i} d^{0} & \in \mathrm{fib}\left(\mathcal{J}_{1} \boldsymbol{E}, V_{\text {Д }}^{*} \boldsymbol{E}\right) .
\end{aligned}
$$

If we put

$$
\breve{\delta}_{0}^{\lambda}=\delta_{0}^{\lambda}+\delta_{i}^{\lambda} x_{0}^{i}, \quad \breve{\delta}_{\mu}^{i}=\delta_{\mu}^{i}-\delta_{\mu}^{0} x_{0}^{i},
$$

then we can write shortly

$$
b_{0}=\breve{\delta}_{0}^{\lambda} \partial_{\lambda}, \quad b_{i}=\left(\delta_{i}^{\lambda}-c \alpha^{0} \tau_{i} \breve{\delta}_{0}^{\lambda}\right) \partial_{\lambda}, \quad \beta^{0}=\left(\delta_{\mu}^{0}+c \alpha^{0} \tau_{i} \breve{\delta}_{\mu}^{i}\right) d^{\mu}, \quad \beta^{i}=\breve{\delta}_{\mu}^{i} d^{\mu} .
$$


We have the inverse relations

$$
\partial_{\lambda}=c \alpha^{0} \tau_{\lambda} b_{0}+\breve{\delta}_{\lambda}^{i} b_{i}, \quad d^{\mu}=\breve{\delta}_{0}^{\mu}\left(\beta^{0}-c \alpha^{0} \tau_{j} \beta^{j}\right)+\delta_{j}^{\mu} \beta^{j} .
$$

4.3. Lemma. We have the equalities

$$
\begin{array}{ll}
\breve{g}_{0 \lambda}=: g\left(b_{0}, \partial_{\lambda}\right)=g_{\rho \lambda} \breve{\delta}_{0}^{\rho}, & \breve{g}^{0 \lambda}=: \bar{g}\left(\beta^{0}, d^{\lambda}\right)=-\left(\alpha^{0}\right)^{2} \breve{\delta}_{0}^{\lambda}, \\
\breve{g}_{i \lambda}=: g\left(b_{i}, \partial_{\lambda}\right)=g_{i \lambda}+\left(\alpha^{0}\right)^{2} \breve{g}_{0 i} \breve{g}_{0 \lambda}, & \breve{g}^{i \lambda}=: \bar{g}\left(\beta^{i}, d^{\lambda}\right)=\breve{\delta}_{\rho}^{i} g^{\rho \lambda}, \\
\hat{g}_{00}=: g\left(b_{0}, b_{0}\right)=-1 /\left(\alpha^{0}\right)^{2}=g_{\rho \sigma} \breve{\delta}_{0}^{\rho} \breve{\delta}_{0}^{\sigma}, & \hat{g}^{00}=: \bar{g}\left(\beta^{0}, \beta^{0}\right)=-\left(\alpha^{0}\right)^{2}, \\
\hat{g}_{i j}=: g\left(b_{i}, b_{j}\right)=g_{i j}+\left(\alpha^{0}\right)^{2} \breve{g}_{0 i} \breve{g}_{0 j}, & \hat{g}^{i j}=: \bar{g}\left(\beta^{i}, \beta^{j}\right)=\breve{\delta}_{\sigma}^{j} \breve{g}^{i \sigma}=\breve{\delta}_{\rho}^{i} \breve{\delta}_{\sigma}^{j} g^{\rho \sigma}, \\
\hat{g}_{0 j}=: g\left(b_{0}, b_{j}\right)=0, & \hat{g}^{0 j}=: \bar{g}\left(\beta^{0}, \beta^{j}\right)=0 .
\end{array}
$$

4.4. Lemma. We have the coordinate expressions

$$
\begin{aligned}
\pi^{\|} & =-\left(\alpha^{0}\right)^{2} \breve{g}_{0 \lambda} \breve{\delta}_{0}^{\mu} d^{\lambda} \otimes \partial_{\mu}, & \pi_{\|} & =-\left(\alpha^{0}\right)^{2} \breve{g}_{0 \lambda} \breve{\delta}_{0}^{\mu} \partial_{\mu} \otimes d^{\lambda}, \\
\pi^{\perp} & =\breve{g}^{i \mu} \breve{g}_{i \lambda} d^{\lambda} \otimes \partial_{\mu}, & \pi_{\perp} & =\breve{g}_{i \lambda} \breve{g}^{i \mu} \partial_{\mu} \otimes d^{\lambda}, \\
g_{\|} & =-\left(\alpha^{0}\right)^{2} \breve{g}_{0 \lambda} \breve{g}_{0 \mu} d^{\lambda} \otimes d^{\mu}, & \bar{g}^{\|} & =-\left(\alpha^{0}\right)^{2} \breve{\delta}_{0}^{\lambda} \breve{\delta}_{0}^{\mu} \partial_{\lambda} \otimes \partial_{\mu}, \\
g_{\perp} & =\left(g_{\lambda \mu}+\left(\alpha^{0}\right)^{2} \breve{g}_{0 \lambda} \breve{g}_{0 \mu}\right) d^{\lambda} \otimes d^{\mu}, & \bar{g}^{\perp} & =\left(g^{\lambda \mu}+\left(\alpha^{0}\right)^{2} \breve{\delta}_{0}^{\lambda} \breve{\delta}_{0}^{\mu}\right) \partial_{\lambda} \otimes \partial_{\mu} .
\end{aligned}
$$

Later, we shall be frequently involved with the following useful technical identities.

4.5. Lemma. We have the following identities

$$
\begin{gathered}
\breve{g}_{0 \lambda} d^{\lambda}=\hat{g}_{00} \beta^{0}, \quad \breve{g}_{i \lambda} d^{\lambda}=\hat{g}_{i j} \beta^{j}, \quad \breve{g}^{0 \lambda} \partial_{\lambda}=\hat{g}^{00} b_{0}, \quad \breve{g}^{i \lambda} \partial_{\lambda}=\hat{g}^{i j} b_{j}, \\
\breve{g}_{i \lambda} \breve{g}^{i \mu}=\delta_{\lambda}^{\mu}+\left(\alpha^{0}\right)^{2} \breve{g}_{0 \lambda} \breve{\delta}_{0}^{\mu}, \quad \breve{g}_{0 i} \breve{g}^{i \lambda}=\breve{\delta}_{0}^{\lambda}-\hat{g}_{00} g^{0 \lambda}, \\
\breve{g}_{i \mu} g^{0 \mu}=\left(\alpha^{0}\right)^{2} \breve{g}_{0 i}, \quad \breve{g}_{0 \nu} \breve{g}^{i \nu}=0, \quad \breve{g}_{i \nu} \breve{g}^{0 \nu}=0, \\
\breve{g}_{i \nu} \breve{\delta}_{0}^{\nu}=0, \quad \breve{g}_{i \mu} \breve{\delta}_{\lambda}^{i}=g_{\lambda \mu}+\left(\alpha^{0}\right)^{2} \breve{g}_{0 \lambda} \breve{g}_{0 \mu}, \quad \breve{g}^{i \lambda} \breve{\delta}_{\lambda}^{j}=\hat{g}^{i j}, \\
\hat{g}^{\lambda \nu} \hat{g}_{\mu \nu}=\hat{g}^{\nu \lambda} \hat{g}_{\nu \mu}=\delta_{\mu}^{\lambda}, \quad \hat{g}^{i h} \hat{g}_{j h}=\hat{g}^{h i} \hat{g}_{h j}=\delta_{j}^{i}, \\
\hat{g}^{i j} g_{j \sigma}=\breve{\delta}_{\sigma}^{i}-\breve{g}^{i 0} \breve{g}_{0 \sigma}, \quad \hat{g}^{i j} \delta_{j}^{\rho}=\breve{g}^{i \rho}-\breve{g}^{i 0} \breve{\delta}_{0}^{\rho}, \quad \breve{g}_{0 \nu} \breve{\delta}_{0}^{\nu}=\hat{g}_{00}, \quad \hat{g}^{i j} \breve{g}_{0 j}=-\hat{g}_{00} \breve{g}^{i 0}, \\
\partial_{i}^{0} \alpha^{0}=\left(\alpha^{0}\right)^{3} \breve{g}_{0 i}, \quad \partial_{\lambda} \alpha^{0}=\frac{1}{2}\left(\alpha^{0}\right)^{3} \partial_{\lambda} \hat{g}_{00} .
\end{gathered}
$$

4.4. Splitting of the spacetime 2-form. Let us consider a linear spacetime connection $K$ and the induced scaled spacetime 2 -forms $\Upsilon=\Upsilon[g, K]$ and $\widetilde{\Upsilon}=\widetilde{\Upsilon}[g, \tilde{K}]$ (see Section 2.4). Then, we can split $\Upsilon$ and $\widetilde{\Upsilon}$, over the phase space, into the parallel and orthogonal components (according to the splitting of $T \boldsymbol{E}$ and $T^{*} \boldsymbol{E}$ achieved in the above Section 4.3) as follows.

4.6. Proposition. The pullback of $\Upsilon$, with respect to $\mathcal{\partial}_{1} \underset{\boldsymbol{E}}{\times} \underset{\boldsymbol{E}}{\times} \boldsymbol{E} \rightarrow T \boldsymbol{E}$, splits as

$$
\Upsilon \equiv \Upsilon_{\|}+\Upsilon_{\perp}
$$


where the parallel and orthogonal components are defined by

$$
\begin{aligned}
& \left.\Upsilon_{\|}=: g\right\lrcorner\left(\nu[K] \wedge \pi_{\|}\right): \mathcal{J}_{1} \underset{\boldsymbol{E}}{\boldsymbol{E}} T \boldsymbol{E} \rightarrow \mathbb{L}^{2} \otimes \Lambda^{2} T^{*} T \boldsymbol{E}, \\
& \left.\Upsilon_{\perp}=: g\right\lrcorner\left(\nu[K] \wedge \pi_{\perp}\right): \mathcal{J}_{1} \boldsymbol{E} \underset{\boldsymbol{E}}{\times} T \boldsymbol{E} \rightarrow \mathbb{L}^{2} \otimes \Lambda^{2} T^{*} T \boldsymbol{E},
\end{aligned}
$$

and have the coordinate expressions

$$
\begin{aligned}
\Upsilon_{\|} & =-c^{2} \tau_{\lambda} \tau_{\mu}\left(\dot{d}^{\lambda}-K_{\nu}{ }_{\rho} \dot{x}^{\rho} d^{\nu}\right) \wedge d^{\mu} \\
& =-\left(\alpha^{0}\right)^{2} \breve{g}_{0 \lambda} \breve{g}_{0 \mu}\left(\dot{d}^{\lambda}-K_{\nu}{ }_{\rho}{ }_{\rho} \dot{x}^{\rho} d^{\nu}\right) \wedge d^{\mu}, \\
\Upsilon_{\perp} & =\left(g_{\lambda \mu}+c^{2} \tau_{\lambda} \tau_{\mu}\right)\left(\dot{d}^{\lambda}-K_{\nu}{ }^{\lambda}{ }_{\rho} \dot{x}^{\rho} d^{\nu}\right) \wedge d^{\mu} \\
& =\left(g_{\lambda \mu}+\left(\alpha^{0}\right)^{2} \breve{g}_{0 \lambda} \breve{g}_{0 \mu}\right)\left(\dot{d}^{\lambda}-K_{\nu}{ }_{\rho} \dot{x}^{\rho} d^{\nu}\right) \wedge d^{\mu} .
\end{aligned}
$$

Proof. It follows from the coordinate expressions of $\pi_{\|}$and $\pi_{\perp}$.

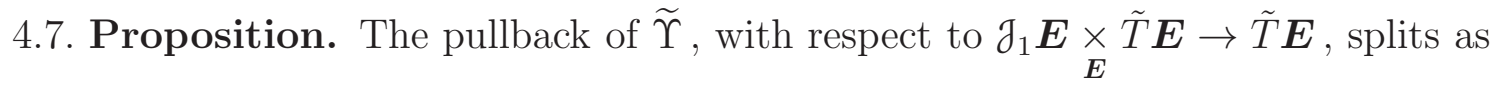

$$
\widetilde{\Upsilon} \equiv \widetilde{\Upsilon}_{\|}+\widetilde{\Upsilon}_{\perp}
$$

where the parallel and orthogonal components are defined by

$$
\begin{aligned}
& \left.\widetilde{\Upsilon}_{\|}=: g\right\lrcorner\left(\nu[\tilde{K}] \wedge \tilde{\pi}_{\|}\right): \mathcal{J}_{1} \boldsymbol{E} \underset{\boldsymbol{E}}{\boldsymbol{E}}\left(\mathbb{T}^{*} \otimes T \boldsymbol{E}\right) \rightarrow\left(\mathbb{T}^{*} \otimes \mathbb{L}^{2}\right) \otimes \Lambda^{2} T^{*} \tilde{T} \boldsymbol{E}, \\
& \left.\widetilde{\Upsilon}_{\perp}=: g\right\lrcorner\left(\nu[\tilde{K}] \wedge \tilde{\pi}_{\perp}\right): \mathcal{\partial}_{1} \boldsymbol{E} \underset{\boldsymbol{E}}{\boldsymbol{T}} \boldsymbol{E} \boldsymbol{E} \rightarrow\left(\mathbb{T}^{*} \otimes \mathbb{L}^{2}\right) \otimes \Lambda^{2} T^{*} \tilde{T} \boldsymbol{E},
\end{aligned}
$$

with $\tilde{\pi}_{\|}=$: id $\otimes \pi_{\|}$and $\tilde{\pi}_{\perp}=$ : id $\otimes \pi_{\perp}$, and have the coordinate expressions

$$
\begin{aligned}
\widetilde{\Upsilon}_{\|} & =-c^{2} \tau_{\lambda} \tau_{\mu} u^{0} \otimes\left(\dot{d}_{0}^{\lambda}-K_{\nu}{ }_{\rho} \dot{x}_{0}^{\rho} d^{\nu}\right) \wedge d^{\mu} \\
& =-\left(\alpha^{0}\right)^{2} \breve{g}_{0 \lambda} \breve{g}_{0 \mu} u^{0} \otimes\left(\dot{d}_{0}^{\lambda}-K_{\nu}{ }_{\rho}{ }_{\rho} \dot{x}_{0}^{\rho} d^{\nu}\right) \wedge d^{\mu}, \\
\widetilde{\Upsilon}_{\perp} & =\left(g_{\lambda \mu}+c^{2} \tau_{\lambda} \tau_{\mu}\right) u^{0} \otimes\left(\dot{d}_{0}^{\lambda}-K_{\nu}{ }^{\lambda}{ }_{\rho} \dot{x}_{0}^{\rho} d^{\nu}\right) \wedge d^{\mu} \\
& =\left(g_{\lambda \mu}+\left(\alpha^{0}\right)^{2} \breve{g}_{0 \lambda} \breve{g}_{0 \mu}\right) u^{0} \otimes\left(\dot{d}_{0}^{\lambda}-K_{\nu}{ }_{\rho}{ }_{\rho} \dot{x}_{0}^{\rho} d^{\nu}\right) \wedge d^{\mu} .
\end{aligned}
$$

4.5. Vertical bundle of the phase space. The metric $g$ yields an isomorphism of the vertical space of the phase space with the $\tau$-vertical subspace of spacetime. This isomorphism can be regarded as analogous to the isomorphism which holds in the case of a fibred manifold.

Let $V \mathcal{J}_{1} \boldsymbol{E} \subset T \mathcal{J}_{1} \boldsymbol{E}$ be the vertical tangent subbundle over $\boldsymbol{E}$. The vertical prolongation of the contact map yields the mutually inverse linear fibred isomorphisms

$$
\nu_{\tau}: \mathcal{J}_{1} \boldsymbol{E} \rightarrow \mathbb{T} \otimes V_{\tau}^{*} \boldsymbol{E} \otimes V \mathcal{J}_{1} \boldsymbol{E} \quad \text { and } \quad \nu_{\tau}^{-1}: \mathcal{J}_{1} \boldsymbol{E} \rightarrow V^{*} \mathcal{J}_{1} \boldsymbol{E} \otimes \mathbb{T}^{*} \otimes V_{\tau} \boldsymbol{E},
$$

with coordinate expressions $\nu_{\tau}=\frac{1}{c_{0} \alpha^{0}} u_{0} \otimes \beta^{i} \otimes \partial_{i}^{0}$ and $\nu_{\tau}^{-1}=c_{0} \alpha^{0} u^{0} \otimes d_{0}^{i} \otimes b_{i}$.

Thus, for each $Y \in \sec \left(\mathcal{J}_{1} \boldsymbol{E}, V \mathcal{J}_{1} \boldsymbol{E}\right)$ and $X \in \sec (\boldsymbol{E}, T \boldsymbol{E})$, we obtain

$$
\nu_{\tau}^{-1}(Y) \in \operatorname{fib}\left(\mathcal{J}_{1} \boldsymbol{E}, \mathbb{T}^{*} \otimes V_{\tau} \boldsymbol{E}\right) \quad \text { and } \quad \nu_{\tau}(X) \in \sec \left(\mathcal{J}_{1} \boldsymbol{E}, \mathbb{T} \otimes V \mathcal{J}_{1} \boldsymbol{E}\right),
$$


with coordinate expressions

$$
\nu_{\tau}^{-1}(Y)=c \alpha^{0} Y_{0}^{i} b_{i} \quad \text { and } \quad \nu_{\tau}(X)=\frac{1}{c \alpha^{0}} \tilde{X}^{i} \partial_{i}^{0}, \quad \text { where } \quad \tilde{X}^{i}=X^{i}-x_{0}^{i} X^{0} .
$$

4.6. Phase connection. Let us introduce the general notion of connection of the phase space and discuss the relation with spacetime connections.

We define a phase connection to be a connection of the bundle $\mathcal{J}_{1} \boldsymbol{E} \rightarrow \boldsymbol{E}$.

A phase connection can be represented, equivalently, by a tangent valued form $\Gamma: \mathcal{J}_{1} \boldsymbol{E} \rightarrow T^{*} \boldsymbol{E} \otimes T \mathcal{J}_{1} \boldsymbol{E}$, which is projectable over 1 $: \boldsymbol{E} \rightarrow T^{*} \boldsymbol{E} \otimes T \boldsymbol{E}$, or by the complementary vertical valued form $\nu[\Gamma]: \mathcal{J}_{1} \boldsymbol{E} \rightarrow T^{*} \mathcal{J}_{1} \boldsymbol{E} \otimes V \mathcal{J}_{1} \boldsymbol{E}$, or by the vector valued form $\nu_{\tau}[\Gamma]=: \nu_{\tau}^{-1} \circ \nu[\Gamma]: \mathcal{\partial}_{1} \boldsymbol{E} \rightarrow T^{*} \mathcal{\partial}_{1} \boldsymbol{E} \otimes\left(\mathbb{T}^{*} \otimes V_{\tau} \boldsymbol{E}\right)$. Their coordinate expressions are

$$
\begin{gathered}
\Gamma=d^{\lambda} \otimes\left(\partial_{\lambda}+\Gamma_{\lambda_{0}}^{i} \partial_{i}^{0}\right), \quad \nu[\Gamma]=\left(d_{0}^{i}-\Gamma_{\lambda_{0}}^{i} d^{\lambda}\right) \otimes \partial_{i}^{0}, \\
\nu_{\tau}[\Gamma]=c \alpha^{0}\left(d_{0}^{i}-\Gamma_{\lambda_{0}}^{i} d^{\lambda}\right) \otimes b_{i}, \quad \text { with } \quad \Gamma_{\lambda_{0}^{i}}^{i} \in \operatorname{map}\left(\mathcal{J}_{1} \boldsymbol{E}, \mathbb{R}\right) .
\end{gathered}
$$

We define the curvature of a phase connection $\Gamma$ to be the vertical valued 2 -form

$$
R[\Gamma]=:-[\Gamma, \Gamma]: \mathcal{J}_{1} \boldsymbol{E} \rightarrow \Lambda^{2} T^{*} \boldsymbol{E} \otimes V \mathcal{J}_{1} \boldsymbol{E},
$$

where $[$,$] is the Frölicher-Nijenhuis bracket. We have the coordinate expression$

$$
R[\Gamma]=-2\left(\partial_{\lambda} \Gamma_{\mu_{0}}^{i}+\Gamma_{\lambda_{0}}^{j} \partial_{j}^{0} \Gamma_{\mu_{0}}^{i}\right) d^{\lambda} \wedge d^{\mu} \otimes \partial_{i}^{0} .
$$

4.8. Theorem. 6] For each linear spacetime connection $K$, there is a unique phase connection $\Gamma$, such that the following diagram commutes

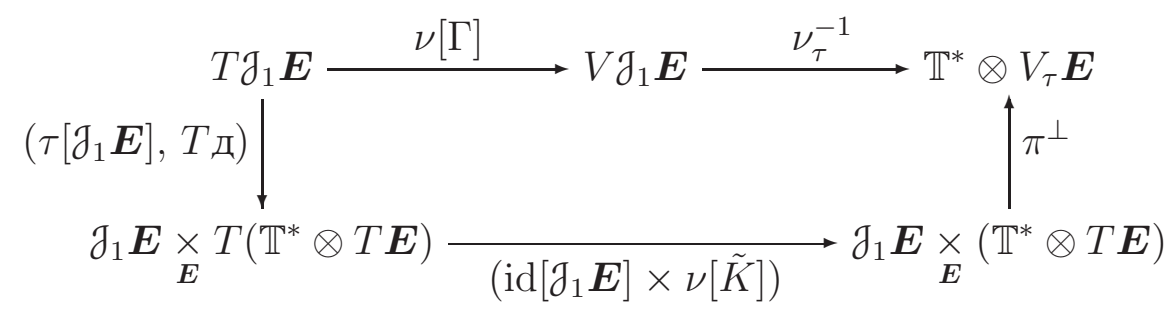

Indeed, we have the coordinate expression

$$
\Gamma_{\lambda_{0}}^{i}=\breve{\delta}_{\rho}^{i} K_{\lambda}^{\rho}{ }_{\sigma} \breve{\delta}_{0}^{\sigma}
$$

Thus, the above correspondence yields a natural map $\chi: K \mapsto \Gamma$ between the set of linear spacetime connections and the set of phase connections.

4.9. Note. We have the following identity [6]

$$
R[\chi(K)]_{\lambda \mu 0}^{i}=\breve{\delta}_{\rho}^{i} R[K]_{\lambda \mu}{ }^{\rho} \sigma \breve{\delta}_{0}^{\sigma} .
$$

4.7. Dynamical phase connection. Let us introduce the general notion of dynamical connection of the phase space and discuss the relation with phase connections and spacetime connections.

Let $\partial_{2} \boldsymbol{E}$ be the space of 2-jets of motions. We can see that this space can be naturally regarded as the affine subundle $\mathcal{\partial}_{2} \boldsymbol{E} \subset \mathbb{T}^{*} \otimes T \mathcal{J}_{1} \boldsymbol{E}$, which projects on д : $\mathcal{J}_{1} \boldsymbol{E} \rightarrow \mathbb{T}^{*} \otimes T \boldsymbol{E}$. 
A dynamical phase connection is defined to be a section $\gamma: \mathcal{J}_{1} \boldsymbol{E} \rightarrow \mathcal{J}_{2} \boldsymbol{E}$, or, equivalently, a section $\gamma: \mathcal{J}_{1} \boldsymbol{E} \rightarrow \mathbb{T}^{*} \otimes T \mathcal{J}_{1} \boldsymbol{E}$, which projects on д. The coordinate expression of a dynamical connection is of the type

$$
\gamma=c \alpha^{0}\left(\partial_{0}+x_{0}^{i} \partial_{i}+\gamma_{00}^{i} \partial_{i}^{0}\right), \quad \text { with } \quad \gamma_{00}^{i} \in \operatorname{map}\left(\mathcal{J}_{1} \boldsymbol{E}, \mathbb{R}\right) .
$$

If $\gamma$ is a dynamical phase connection, then we have $\gamma\lrcorner \tau=1$.

If $\Gamma$ is a phase connection, then the section $\gamma=: \gamma[\Gamma]=:$ д $\lrcorner$ : $\mathcal{J}_{1} \boldsymbol{E} \rightarrow \mathbb{T}^{*} \otimes T \mathcal{J}_{1} \boldsymbol{E}$ turns out to be a dynamical phase connection, whose coordinate expression is given by

$$
\gamma_{00}^{i}=\Gamma_{00}^{i}+\Gamma_{j 0}^{i} x_{0}^{j}=\Gamma_{\rho 0}^{i} \breve{\delta}_{0}^{\rho} .
$$

Hence, a linear spacetime connection $K$ yields the dynamical phase connection $\gamma=: \gamma[K]=:$ д $\lrcorner \chi(K)$. Its coordinate expression is

$$
\gamma_{00}^{i}=\breve{\delta}_{\rho}^{i} K_{\sigma}{ }^{\rho}{ }_{\tau} \breve{\delta}_{0}^{\sigma} \breve{\delta}_{0}^{\tau}
$$

4.8. Phase 2-form and 2-vector. Let us introduce the general notions a phase 2forms and phase 2-vectors associated with a phase connection and discuss the relation with spacetime connections.

If $\Gamma$ is a phase connection, then we define the scaled phase 2-form and the scaled phase 2-vector associated with $g$ and $\Gamma$ to be, respectively, the sections

$$
\begin{aligned}
& \Omega=: \Omega[g, \Gamma]=: g\lrcorner\left(\nu_{\tau}[\Gamma] \wedge \theta\right): \mathcal{J}_{1} \boldsymbol{E} \rightarrow\left(\mathbb{T}^{*} \otimes \mathbb{L}^{2}\right) \otimes \Lambda^{2} T^{*} \mathcal{J}_{1} \boldsymbol{E}, \\
& \Lambda=: \Lambda[g, \Gamma]=: \bar{g}\lrcorner\left(\Gamma \wedge \nu_{\tau}\right) \quad: \mathcal{J}_{1} \boldsymbol{E} \rightarrow\left(\mathbb{T} \otimes \mathbb{L}^{-2}\right) \otimes \Lambda^{2} T \mathcal{J}_{1} \boldsymbol{E} .
\end{aligned}
$$

Their coordinate expressions are

$$
\Omega=c \alpha^{0} \breve{g}_{i \mu}\left(d_{0}^{i}-\Gamma_{\lambda_{0}}^{i} d^{\lambda}\right) \wedge d^{\mu} \quad \text { and } \quad \Lambda=\frac{1}{c \alpha^{0}} \breve{g}^{j \lambda}\left(\partial_{\lambda}+\Gamma_{\lambda_{0}}^{i} \partial_{i}^{0}\right) \wedge \partial_{j}^{0} .
$$

There is a unique dynamical phase connection $\bar{\gamma}$, such that $\bar{\gamma}\lrcorner \Omega[g, \Gamma]=0$. Namely, $\bar{\gamma}=\gamma[\Gamma]: \mathcal{J}_{1} \boldsymbol{E} \rightarrow \mathbb{T}^{*} \otimes T \mathcal{J}_{1} \boldsymbol{E}$.

4.10. Lemma. If $\Gamma$ is a phase connection, then we have $i_{\Lambda} \Omega=-3$.

Proof. We have $i_{\Lambda} \Omega=-\breve{g}^{i \mu} \breve{g}_{i \mu}=-3$.

Thus, a linear spacetime connection $K$ yields the scaled phase 2 -form and the scaled phase 2-vector

$$
\Omega=: \Omega[g, K]=: \Omega[g, \chi(K)] \quad \text { and } \quad \Lambda=: \Lambda[g, K]=: \Lambda[g, \chi(K)] .
$$

Their coordinate expressions are

$$
\begin{aligned}
& \Omega=c \alpha^{0} \breve{g}_{i \mu}\left(d_{0}^{i}-\breve{\delta}_{\rho}^{i} K_{\lambda}{ }^{\rho}{ }_{\sigma} \breve{\delta}_{0}^{\sigma} d^{\lambda}\right) \wedge d^{\mu}, \\
& \Lambda=\frac{1}{c \alpha^{0}} \breve{g}^{j \lambda}\left(\partial_{\lambda}+\breve{\delta}_{\rho}^{i} K_{\lambda}{ }^{\rho}{ }_{\sigma} \breve{\delta}_{0}^{\sigma} \partial_{i}^{0}\right) \wedge \partial_{j}^{0} .
\end{aligned}
$$


4.9. Spacetime and phase $\mathbf{2}$-forms and $\mathbf{2}$-vectors. Let $K$ be a linear spacetime connection. Then, we can compare the scaled phase 2-form $\Omega=: \Omega[g, K]$ and the scaled phase 2-vector $\Lambda=: \Lambda[g, K]$ with the scaled spacetime 2 -form $\widetilde{\Upsilon}=: \widetilde{\Upsilon}[g, \tilde{K}]$ and the scaled spacetime 2 -vector $\widetilde{\Xi}=: \widetilde{\Xi}[g, \tilde{K}]$, respectively, in the following way.

4.11. Proposition. The contact map д yields the following scaled 2 -forms of $\mathcal{J}_{1} \boldsymbol{E}$

$$
\begin{aligned}
& \text { д } \widetilde{\Upsilon}: \partial_{1} \boldsymbol{E} \rightarrow\left(\mathbb{T}^{*} \otimes \mathbb{L}^{2}\right) \otimes \Lambda^{2} T^{*} \partial_{1} \boldsymbol{E}, \\
& \text { д* } \widetilde{\Upsilon}_{\|}: \partial_{1} \boldsymbol{E} \rightarrow\left(\mathbb{T}^{*} \otimes \mathbb{L}^{2}\right) \otimes \Lambda^{2} T^{*} \partial_{1} \boldsymbol{E}, \\
& \text { д }^{*} \widetilde{\Upsilon}_{\perp}: \partial_{1} \boldsymbol{E} \rightarrow\left(\mathbb{T}^{*} \otimes \mathbb{L}^{2}\right) \otimes \Lambda^{2} T^{*} \partial_{1} \boldsymbol{E},
\end{aligned}
$$

which fulfill the equality

$$
\text { д }^{*} \widetilde{\Upsilon}=\text { д }^{*} \widetilde{\Upsilon}_{\|}+\text {д }^{*} \widetilde{\Upsilon}_{\perp} \text {. }
$$

We have the coordinate expressions

$$
\begin{aligned}
\text { д }^{*} \widetilde{\Upsilon} & =c \alpha^{0}\left(\breve{g}_{i \mu} d_{0}^{i}+\left(\frac{1}{2}\left(\alpha^{0}\right)^{2} \breve{g}_{0 \mu} \partial_{\nu} \hat{g}_{00}-g_{\lambda \mu} \breve{\delta}_{0}^{\rho} K_{\nu}{ }^{\lambda}{ }_{\rho}\right) d^{\nu}\right) \wedge d^{\mu}, \\
\text { д }^{*} \widetilde{\Upsilon}_{\|} & =c\left(\alpha^{0}\right)^{3} \breve{g}_{0 \mu}\left(\frac{1}{2} \partial_{\nu} \hat{g}_{00}+\breve{g}_{0 \lambda} \breve{\delta}_{0}^{\rho} K_{\nu}{ }^{\lambda} \rho\right) d^{\nu} \wedge d^{\mu}, \\
\text { д*}^{*} \widetilde{\Upsilon}_{\perp} & =c \alpha^{0} \breve{g}_{i \mu}\left(d_{0}^{i}-\breve{\delta}_{\lambda}^{i} \breve{\delta}_{0}^{\rho} K_{\nu}{ }^{\lambda}{ }_{\rho} d^{\nu}\right) \wedge d^{\mu} .
\end{aligned}
$$

Proof. The equality

$$
\left(x^{\lambda}, \dot{x}_{0}^{0}, \dot{x}_{0}^{i}\right) \text { о д }=\left(x^{\lambda}, c_{0} \alpha^{0}, c_{0} \alpha^{0} x_{0}^{i}\right)
$$

yields

$$
\text { д }^{*} \dot{x}_{0}^{0}=c_{0} \alpha^{0}, \quad \text { д }{ }^{*} \dot{x}_{0}^{i}=c_{0} \alpha^{0} x_{0}^{i}
$$

and

$$
\begin{aligned}
\text { म }^{*} \dot{d}_{0}^{0} & =c_{0} \partial_{\lambda} \alpha^{0} d^{\lambda}+c_{0} \partial_{j}^{0} \alpha^{0} d_{0}^{j} \\
& =\frac{1}{2} c_{0}\left(\alpha^{0}\right)^{3} \partial_{\lambda} \hat{g}_{00} d^{\lambda}+c_{0}\left(\alpha^{0}\right)^{3} \breve{g}_{0 j} d_{0}^{j} \\
\text { म }^{*} \dot{d}_{0}^{i} & =c_{0} \partial_{\lambda} \alpha^{0} x_{0}^{i} d^{\lambda}+c_{0} \partial_{j}^{0} \alpha^{0} x_{0}^{i} d_{0}^{j}+c_{0} \alpha^{0} d_{0}^{i} \\
& =\frac{1}{2} c_{0}\left(\alpha^{0}\right)^{3} \partial_{\lambda}\left(\hat{g}_{00}\right) x_{0}^{i} d^{\lambda}+c_{0}\left(\alpha^{0}\right)^{3} \breve{g}_{0 j} x_{0}^{i} d_{0}^{j}+c_{0} \alpha^{0} d_{0}^{i} .
\end{aligned}
$$

Then, we obtain

$$
\begin{aligned}
\text { д }^{*} \widetilde{\Upsilon}= & \text { д}^{*}\left(g_{\lambda \mu} u^{0} \otimes\left(\dot{d}_{0}^{\lambda}-K_{\nu}{ }_{\rho}{ }_{\rho} \dot{x}_{0}^{\rho} d^{\nu}\right) \wedge d^{\mu}\right) \\
= & g_{0 \mu} u^{0} \otimes\left(\frac{1}{2} c_{0}\left(\alpha^{0}\right)^{3} \partial_{\lambda} \hat{g}_{00} d^{\lambda}+c_{0}\left(\alpha^{0}\right)^{3} \breve{g}_{0 i} d_{0}^{i}\right) \wedge d^{\mu} \\
& +g_{j \mu} u^{0} \otimes\left(\frac{1}{2} c_{0}\left(\alpha^{0}\right)^{3} \partial_{\lambda} \hat{g}_{00} x_{0}^{j} d^{\lambda}+c_{0}\left(\alpha^{0}\right)^{3} \breve{g}_{0 i} x_{0}^{j} d_{0}^{i}+c_{0} \alpha^{0} d_{0}^{j}\right) \wedge d^{\mu} \\
& -c_{0} \alpha^{0} g_{\lambda \mu}\left(K_{\nu}{ }^{\lambda}{ }_{0}+K_{\nu}{ }^{\lambda}{ }_{j} x_{0}^{j}\right) u^{0} \otimes d^{\nu} \wedge d^{\mu} \\
= & c^{0} \alpha^{0} \breve{g}_{i \mu} u^{0} \otimes d_{0}^{i} \wedge d^{\mu}+\frac{1}{2} c_{0}\left(\alpha^{0}\right)^{3} \breve{g}_{0 \mu} \partial_{\lambda} \hat{g}_{00} u^{0} \otimes d^{\lambda} \wedge d^{\mu} \\
& -c_{0} \alpha^{0} g_{\lambda \mu}\left(K_{\nu}{ }^{\lambda}{ }_{0}+K_{\nu}{ }_{j}{ }_{j} x_{0}^{j}\right) u^{0} \otimes d^{\nu} \wedge d^{\mu} .
\end{aligned}
$$


Moreover, by recalling the equality $\left(\alpha^{0}\right)^{2}\left(\breve{g}_{00}+\breve{g}_{0 i} x_{0}^{i}\right)=-1$, we obtain

$$
\begin{aligned}
\text { д }^{*} \widetilde{\Upsilon}_{\|}= & - \text {д }^{*}\left(\left(\alpha^{0}\right)^{2} \breve{g}_{0 \lambda} \breve{g}_{0 \mu} u^{0} \otimes\left(\dot{d}_{0}^{\lambda}-K_{\nu}{ }_{\rho}{ }_{\rho} \dot{x}_{0}^{\rho} d^{\nu}\right) \wedge d^{\mu}\right) \\
= & -\left(\alpha^{0}\right)^{2} \breve{g}_{00} \breve{g}_{0 \mu}\left(\frac{1}{2} c_{0}\left(\alpha^{0}\right)^{3} \partial_{\lambda} \hat{g}_{00} d^{\lambda}+c_{0}\left(\alpha^{0}\right)^{3} \breve{g}_{0 j} d_{0}^{j}\right) \wedge d^{\mu} \\
& -\left(\alpha^{0}\right)^{2} \breve{g}_{0 i} \breve{g}_{0 \mu} u^{0} \otimes\left(\frac{1}{2} c_{0}\left(\alpha^{0}\right)^{3} \partial_{\lambda} \hat{g}_{00} x_{0}^{i} d^{\lambda}+c_{0}\left(\alpha^{0}\right)^{3} \breve{g}_{0 j} x_{0}^{i} d_{0}^{j}+c_{0} \alpha^{0} d_{0}^{i}\right) \wedge d^{\mu} \\
& +c_{0}\left(\alpha^{0}\right)^{3} \breve{g}_{0 \lambda} \breve{g}_{0 \mu}\left(K_{\nu}{ }^{\lambda}{ }_{0}+K_{\nu}{ }^{\lambda}{ }_{j} x_{0}^{j}\right) u^{0} \otimes d^{\nu} \wedge d^{\mu} \\
= & \frac{1}{2} c_{0}\left(\alpha^{0}\right)^{3} \breve{g}_{0 \mu} \partial_{\lambda} \hat{g}_{00} u^{0} \otimes d^{\lambda} \wedge d^{\mu}+c^{0}\left(\alpha^{0}\right)^{3} \breve{g}_{0 \lambda} \breve{g}_{0 \mu} K_{\nu}{ }^{\lambda}{ }_{\rho} \breve{\delta}_{0}^{\rho} u^{0} \otimes d^{\nu} \wedge d^{\mu} .
\end{aligned}
$$

Finally, we obtain

$$
\begin{aligned}
\text { д }^{*} \widetilde{\Upsilon}_{\perp} & =\text { д }^{*} \widetilde{\Upsilon}-\text { д }^{*} \widetilde{\Upsilon}_{\|} \\
& =c_{0} \alpha^{0} \breve{g}_{i \mu} u^{0} \otimes\left(d_{0}^{i}-\breve{\delta}_{\rho}^{i} K_{\nu}{ }^{\rho}{ }_{\sigma} \breve{\delta}_{0}^{\sigma} d^{\nu}\right) \wedge d^{\mu} .
\end{aligned}
$$

4.12. Theorem. We have

$$
\Omega=\text { д }^{*} \widetilde{\Upsilon}_{\perp}
$$

Proof. The Theorem follows by a direct comparison of the coordinate expressions

$$
\begin{gathered}
\Omega=c \alpha^{0} \breve{g}_{i \mu}\left(d_{0}^{i}-\Gamma_{\lambda 0}^{i} d^{\lambda}\right) \wedge d^{\mu}, \\
\text { म }^{*} \widetilde{\Upsilon}_{\perp}=c \alpha^{0} \breve{g}_{i \mu}\left(d_{0}^{i}-\breve{\delta}_{\lambda}^{i} K_{\nu}^{\lambda} \breve{\delta}_{0}^{\rho} d^{\nu}\right) \wedge d^{\mu},
\end{gathered}
$$

where we put $\Gamma_{\nu 0}^{i}=\breve{\delta}_{\lambda}^{i} K_{\nu}{ }_{\rho}^{\lambda} \breve{\delta}_{0}^{\rho}$.

4.13. Corollary. $\Lambda$ is the unique scaled phase 2 -vector such that the following diagram commutes

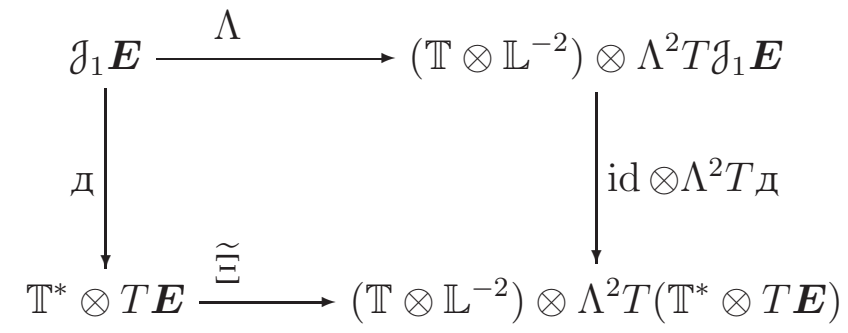

Proof. We have

$$
\widetilde{\Xi} \circ \text { д }=g^{\lambda \mu} u_{0} \otimes\left(\partial_{\lambda}+c_{0} \alpha^{0} K_{\lambda}^{\nu}{ }_{\rho} \breve{\delta}_{0}^{\rho} \dot{\partial}_{\nu}^{0}\right) \wedge \dot{\partial}_{\mu}^{0}
$$

and

$$
\begin{aligned}
\left(\mathrm{id} \otimes \Lambda^{2} T \text { д }\right) \circ \Lambda= & g^{\lambda \mu} u_{0} \otimes \partial_{\lambda} \wedge \dot{\partial}_{\mu}^{0} \\
& +c_{0} \alpha^{0}\left(\left(\alpha^{0}\right)^{2}\left(\frac{1}{2} \breve{g}^{j \lambda} \partial_{\lambda} \hat{g}_{00} \breve{\delta}_{0}^{\nu} \delta_{j}^{\mu}+\breve{\delta}_{0}^{\lambda} \Gamma_{\lambda_{0}}^{i} \delta_{i}^{\nu} \breve{\delta}_{0}^{\mu}+\breve{g}^{j \lambda} \Gamma_{\lambda_{0}}^{i} \breve{g}_{0 i} \delta_{j}^{\mu} \breve{\delta}_{0}^{\nu}\right)\right. \\
& \left.+g^{0 \lambda} \Gamma_{\lambda 0}^{i} \delta_{i}^{\nu} \breve{\delta}_{0}^{\mu}+\breve{g}^{j \lambda} \Gamma_{\lambda_{0}}^{i} \delta_{j}^{\mu} \delta_{i}^{\nu}\right) \dot{\partial}_{\nu}^{0} \wedge \dot{\partial}_{\mu}^{0} .
\end{aligned}
$$

By comparing the coefficients standing by $u_{0} \otimes \dot{\partial}_{0} \wedge \dot{\partial}_{j}$ and $u_{0} \otimes \dot{\partial}_{i} \wedge \dot{\partial}_{j}$, we get

$$
\left(g^{j \lambda} K_{\lambda}{ }^{0}{ }_{\rho}-g^{0 \lambda} K_{\lambda}{ }^{j}{ }_{\rho}\right) \breve{\delta}_{0}^{\rho}=\left(\alpha^{0}\right)^{2}\left(\frac{1}{2} g^{j \lambda} \partial_{\lambda} \hat{g}_{00}-\breve{\delta}_{0}^{\lambda} \Gamma_{\lambda_{0}}^{j}+\breve{g}^{j \lambda} \Gamma_{\lambda_{0}}^{p} \breve{g}_{0 p}\right)-g^{0 \lambda} \Gamma_{\lambda_{0}}^{j}
$$


and

$$
\begin{aligned}
\left(g^{j \lambda} K_{\lambda}^{j}{ }_{\rho}-g^{i \lambda} K_{\lambda}^{j}{ }_{\rho}\right) \breve{\delta}_{0}^{\rho}= & x_{0}^{i}\left[\left(\alpha^{0}\right)^{2}\left(\frac{1}{2} g^{j \lambda} \partial_{\lambda} \hat{g}_{00}-\breve{\delta}_{0}^{\lambda} \Gamma_{\lambda 0}^{j}+\breve{g}^{j \lambda} \Gamma_{\lambda 0}^{p} \breve{g}_{0 p}\right)-g^{0 \lambda} \Gamma_{\lambda_{0}}^{j}\right] \\
& -x_{0}^{j}\left[\left(\alpha^{0}\right)^{2}\left(\frac{1}{2} g^{i \lambda} \partial_{\lambda} \hat{g}_{00}-\breve{\delta}_{0}^{\lambda} \Gamma_{\lambda_{0}}^{i}+\breve{g}^{i \lambda} \Gamma_{\lambda_{0}}^{p} \breve{g}_{0 p}\right)-g^{0 \lambda} \Gamma_{\lambda_{0}}^{i}\right] \\
& +\breve{g}^{j \lambda} \Gamma_{\lambda_{0}}^{i}-\breve{g}^{i \lambda} \Gamma_{\lambda_{0}}^{j},
\end{aligned}
$$

respectively.

Then, inserting the first equality into the second one, we get

$$
\begin{aligned}
\breve{g}^{j \lambda} \Gamma_{\lambda 0}^{i}-\breve{g}^{i \lambda} \Gamma_{\lambda 0}^{j}= & \left(g^{j \lambda} K_{\lambda}{ }^{i}{ }_{\rho}-g^{i \lambda} K_{\lambda}{ }^{j}{ }_{\rho}\right) \breve{\delta}_{0}^{\rho} \\
& \left.\left.-x_{0}^{i}\left(g^{j \lambda} K_{\lambda}{ }^{0}{ }_{\rho}-g^{0 \lambda} K_{\lambda^{j}}{ }^{j}\right)\right) \breve{\delta}_{0}^{\rho}+x_{0}^{j}\left(g^{i \lambda} K_{\lambda}{ }^{0}{ }_{\rho}-g^{0 \lambda} K_{\lambda}{ }^{i}{ }_{\rho}\right)\right) \breve{\delta}_{0}^{\rho}
\end{aligned}
$$

which is satisfied if and only if $\Gamma_{\lambda_{0}}^{i}=\breve{\delta}_{\nu}^{i} K_{\lambda}{ }_{\rho}{ }_{\rho} \breve{\delta}_{0}^{\rho}$, i.e., if and only if $\Gamma=\chi(K)$.

\section{Contact and Jacobi structures: general CASE}

In this section, we consider the metric $g$, a general phase connection $\Gamma$ and the induced objects $\Omega=: \Omega[g, \Gamma], \Lambda=: \Lambda[g, \Gamma], \gamma=: \gamma[\Gamma]$. Then, we analyse the conditions by which $g$ and $\Gamma$ induce scaled almost-cosymplectic-contact, or contact, structures and scaled almost-coPoisson-Jacobi, or Jacobi, structures (see Introduction) on the phase space.

5.1. Regularity and duality. Let us analyse the non-degeneracy of the scaled covariant pair $\left(-c^{2} \tau, \Omega\right)$ and of the scaled contravariant pair $\left(-\frac{1}{c^{2}} \gamma, \Lambda\right)$ and the condition for their duality [8].

5.1. Lemma. The section $-c^{2} \tau \wedge \Omega \wedge \Omega \wedge \Omega: \mathcal{J}_{1} \boldsymbol{E} \rightarrow\left(\mathbb{T}^{* 4} \otimes \mathbb{L}^{8}\right) \otimes \Lambda^{7} T^{*} \mathcal{J}_{1} \boldsymbol{E}$ is a scaled volume form, with coordinate expression

$$
-c^{2} \tau \wedge \Omega \wedge \Omega \wedge \Omega=3 ! c^{4}\left(\alpha^{0}\right)^{4}|g| d_{0}^{1} \wedge d_{0}^{2} \wedge d_{0}^{3} \wedge d^{0} \wedge d^{1} \wedge d^{2} \wedge d^{3} .
$$

Hence, the pair $\left(-c^{2} \tau, \Omega\right)$ is a regular covariant pair.

Proof. The equalities $\tau=\tau_{\lambda} d^{\lambda}=-\frac{\alpha^{0}}{c} \breve{g}_{0 \lambda} d^{\lambda}$ and $\Omega=c \alpha^{0} \breve{g}_{i \mu}\left(d_{0}^{i}-\Gamma_{\nu 0}^{i} d^{\nu}\right) \wedge d^{\mu}$ yield

$$
-c^{2} \tau \wedge \Omega \wedge \Omega \wedge \Omega=-c^{5}\left(\alpha^{0}\right)^{3} \tau_{\lambda} \breve{g}_{i_{1} \mu_{1}} \breve{g}_{i_{2} \mu_{2}} \breve{g}_{i_{3} \mu_{3}} d^{\lambda} \wedge d_{0}^{i_{1}} \wedge d^{\mu_{1}} \wedge d_{0}^{i_{2}} \wedge d^{\mu_{2}} \wedge d_{0}^{i_{3}} \wedge d^{\mu_{3}} .
$$

Hence, by taking into account that the antisymmetrisation makes some terms vanishing, we obtain

$$
\begin{aligned}
-c^{2} \tau \wedge \Omega^{3} & =c^{4}\left(\alpha^{0}\right)^{4} \breve{g}_{0 \lambda} g_{i_{1} \mu_{1}} g_{i_{2} \mu_{2}} g_{i_{3} \mu_{3}} d^{\lambda} \wedge d_{0}^{i_{1}} \wedge d^{\mu_{1}} \wedge d_{0}^{i_{2}} \wedge d^{\mu_{2}} \wedge d_{0}^{i_{3}} \wedge d^{\mu_{3}} \\
& =(3 !) c^{4}\left(\alpha^{0}\right)^{4} g_{0 \lambda} g_{1 \mu_{1}} g_{2 \mu_{2}} g_{3 \mu_{3}} d^{\lambda} \wedge d_{0}^{1} \wedge d^{\mu_{1}} \wedge d_{0}^{2} \wedge d^{\mu_{2}} \wedge d_{0}^{3} \wedge d^{\mu_{3}} \\
& =(3 !) c^{4}\left(\alpha^{0}\right)^{4} g_{0 \lambda} g_{1 \mu_{1}} g_{2 \mu_{2}} g_{3 \mu_{3}} d^{\lambda} \wedge d_{0}^{1} \wedge d^{\mu_{1}} \wedge d_{0}^{2} \wedge d^{\mu_{2}} \wedge d_{0}^{3} \wedge d^{\mu_{3}} \\
& =(3 !) c^{4}\left(\alpha^{0}\right)^{4}|g| d^{0} \wedge d_{0}^{1} \wedge d^{1} \wedge d_{0}^{2} \wedge d^{2} \wedge d_{0}^{3} \wedge d^{3} .
\end{aligned}
$$

5.2. Lemma. The section $-\frac{1}{c^{2}} \gamma \wedge \Lambda \wedge \Lambda \wedge \Lambda: \mathcal{J}_{1} \boldsymbol{E} \rightarrow\left(\mathbb{T}^{4} \otimes \mathbb{L}^{-8}\right) \otimes \Lambda^{7} T \mathcal{J}_{1} \boldsymbol{E}$ is a scaled volume vector, with coordinate expression

$$
-\frac{1}{c^{2}} \gamma \wedge \Lambda \wedge \Lambda \wedge \Lambda=-3 ! \frac{1}{\left(c \alpha^{0}\right)^{4}}|\bar{g}| \partial_{1}^{0} \wedge \partial_{2}^{0} \wedge \partial_{3}^{0} \wedge \partial_{0} \wedge \partial_{1} \wedge \partial_{2} \wedge \partial_{3}
$$


Hence, the pair $\left(-\frac{1}{c^{2}} \gamma, \Lambda\right)$ is a regular contravariant pair.

Proof. The equalities $\gamma=c \alpha^{0}\left(\breve{\delta}_{0}^{\lambda} \partial_{\lambda}+\gamma_{00}^{i} \partial_{i}^{0}\right)$ and $\Lambda=\frac{1}{c \alpha^{0}} \breve{g}^{j \mu}\left(\partial_{\mu}+\Gamma_{\mu 0}^{i} \partial_{i}^{0}\right) \wedge \partial_{j}^{0}$ yield

$$
\begin{aligned}
-\frac{1}{c^{2}} \gamma \wedge \Lambda^{3} & =-\frac{1}{c^{4}\left(\alpha^{0}\right)^{2}} \breve{g}^{i_{1} \mu_{1}} \breve{g}^{i_{2} \mu_{2}} \breve{g}^{i_{3} \mu_{3}}\left(\breve{\delta}_{0}^{\lambda} \partial_{\lambda}+\gamma_{00}^{i} \partial_{i_{0}}^{0}\right) \wedge \partial_{\mu_{1}} \wedge \partial_{i_{1}}^{0} \wedge \partial_{\mu_{2}} \wedge \partial_{i_{2}}^{0} \wedge \partial_{\mu_{3}} \wedge \partial_{i_{3}}^{0} \\
& =-\frac{1}{c^{4}\left(\alpha^{0}\right)^{2}} \breve{g}^{i_{1} \mu_{1}} \breve{g}^{i_{2} \mu_{2}} \breve{g}^{i_{3} \mu_{3}} \breve{\delta}_{0}^{\lambda} \partial_{\lambda} \wedge \partial_{\mu_{1}} \wedge \partial_{i_{1}}^{0} \wedge \partial_{\mu_{2}} \wedge \partial_{i_{2}}^{0} \wedge \partial_{\mu_{3}} \wedge \partial_{i_{3}}^{0} .
\end{aligned}
$$

Hence, by the identity $\breve{\delta}_{0}^{\lambda}=\breve{g}_{0 i} \breve{g}^{i \lambda}-\frac{1}{\left(\alpha^{0}\right)^{2}} g^{0 \lambda}$ and the fact that the antisymmetrisation makes some terms vanishing, we obtain

$$
\begin{aligned}
-\frac{1}{c^{2}} \gamma \wedge \Lambda^{3}= & -\frac{1}{c^{4}\left(\alpha^{0}\right)^{2}} \breve{g}^{i_{1} \mu_{1}} \breve{g}^{i_{2} \mu_{2}} \breve{g}^{i_{3} \mu_{3}}\left(\breve{g}_{0 i} \breve{g}^{i \lambda}-\frac{1}{\left(\alpha^{0}\right)^{2}} g^{0 \lambda}\right) \\
& \partial_{\lambda} \wedge \partial_{\mu_{1}} \wedge \partial_{i_{1}}^{0} \wedge \partial_{\mu_{2}} \wedge \partial_{i_{2}}^{0} \wedge \partial_{\mu_{3}} \wedge \partial_{i_{3}}^{0} \\
& =\frac{1}{\left(c \alpha^{0}\right)^{4}} \breve{g}^{i_{1} \mu_{1}} \breve{g}^{i_{2} \mu_{2}} \breve{g}^{i_{3} \mu_{3}} g^{0 \lambda} \partial_{\lambda} \wedge \partial_{\mu_{1}} \wedge \partial_{i_{1}}^{0} \wedge \partial_{\mu_{2}} \wedge \partial_{i_{2}}^{0} \wedge \partial_{\mu_{3}} \wedge \partial_{i_{3}}^{0} \\
& =\frac{1}{\left(c \alpha^{0}\right)^{4}} g^{i_{1} \mu_{1}} g^{i_{2} \mu_{2}} g^{i_{3} \mu_{3}} g^{0 \lambda} \partial_{\lambda} \wedge \partial_{\mu_{1}} \wedge \partial_{i_{1}}^{0} \wedge \partial_{\mu_{2}} \wedge \partial_{i_{2}}^{0} \wedge \partial_{\mu_{3}} \wedge \partial_{i_{3}}^{0} \\
& =3 ! \frac{1}{\left(c \alpha^{0}\right)^{4}} g^{0 \lambda} g^{1 \mu_{1}} g^{2 \mu_{2}} g^{3 \mu_{3}} \partial_{\lambda} \wedge \partial_{\mu_{1}} \wedge \partial_{1}^{0} \wedge \partial_{\mu_{2}} \wedge \partial_{2}^{0} \wedge \partial_{\mu_{3}} \wedge \partial_{3}^{0} \\
& =3 ! \frac{1}{\left(c \alpha^{0}\right)^{4}}|\bar{g}| \partial_{0} \wedge \partial_{1} \wedge \partial_{1}^{0} \wedge \partial_{2} \wedge \partial_{2}^{0} \wedge \partial_{3} \wedge \partial_{3}^{0} \\
& =-3 ! \frac{1}{\left(c \alpha^{0}\right)^{4}}|\bar{g}| \partial_{1}^{0} \wedge \partial_{2}^{0} \wedge \partial_{3}^{0} \wedge \partial_{0} \wedge \partial_{1} \wedge \partial_{2} \wedge \partial_{3} .
\end{aligned}
$$

5.3. Lemma. We have

$$
\left(\Lambda^{\sharp} \otimes \Lambda^{\sharp}\right)(\Omega)=-\Lambda, \quad\left(\Omega^{b} \otimes \Omega^{b}\right)(\Lambda)=-\Omega .
$$

Proof. We have

$$
\Lambda^{\sharp}\left(d^{\lambda}\right)=\frac{1}{c \alpha^{0}} \breve{g}^{j \lambda} \partial_{j}^{0}, \quad \Lambda^{\sharp}\left(d_{0}^{i}\right)=\frac{1}{c \alpha^{0}}\left(-\breve{g}^{i \mu} \partial_{\mu}+\left(\breve{g}^{j \rho} \Gamma_{\rho_{0}}^{i}-\breve{g}^{i \rho} \Gamma_{\rho_{0}}^{j}\right) \partial_{j}^{0}\right)
$$

and

$$
\Omega^{b}\left(\partial_{\lambda}\right)=c \alpha^{0}\left(-\breve{g}_{i \lambda} d_{0}^{i}+\left(\breve{g}_{p \lambda} \Gamma_{\mu_{0}}^{p}-\breve{g}_{p \mu} \Gamma_{\lambda_{0}}^{p}\right) d^{\mu}\right), \quad \Omega^{b}\left(\partial_{i}^{0}\right)=c \alpha^{0} \breve{g}_{i \mu} d^{\mu}
$$

Then

$$
\begin{aligned}
\left(\Lambda^{\sharp} \otimes \Lambda^{\sharp}\right)(\Omega) & =c \alpha^{0}\left(\Lambda^{\sharp}\left(d_{0}^{i}\right)-\Gamma_{\nu 0}^{i} \Lambda^{\sharp}\left(d^{\nu}\right)\right) \wedge \Lambda^{\sharp}\left(d^{\mu}\right) \\
& =\frac{1}{c \alpha^{0}} \breve{g}_{i \mu}\left(-\breve{g}^{i \lambda} \partial_{\lambda}+\left(\breve{g}^{j \rho} \Gamma_{\rho_{0}}^{i}-\breve{g}^{i \rho} \Gamma_{\rho_{0}}^{j}\right) \partial_{j}^{0}-\breve{g}^{j \rho} \Gamma_{\rho_{0}}^{i} \partial_{j}^{0}\right) \wedge\left(\breve{g}^{k \mu} \partial_{k}^{0}\right) \\
& =\frac{1}{c \alpha^{0}}\left(-\breve{g}^{i \lambda} \partial_{\lambda}-\breve{g}^{i \lambda} \Gamma_{\lambda_{0}}^{j} \partial_{j}^{0}\right) \wedge \partial_{i}^{0}
\end{aligned}
$$

and

$$
\begin{aligned}
\left(\Omega^{b} \otimes \Omega^{b}\right)(\Lambda) & =\frac{1}{c \alpha^{0}} \breve{g}^{j \lambda}\left(\Omega^{b}\left(\partial_{\lambda}\right)+\Gamma_{\lambda_{0}}^{i} \Omega^{b}\left(\partial_{i}^{0}\right)\right) \wedge \Omega^{b}\left(\partial_{j}^{0}\right) \\
& =c \alpha^{0} \breve{g}^{i \lambda}\left(-\breve{g}_{p \lambda} d_{0}^{p}+\left(\breve{g}_{p \lambda} \Gamma_{\mu 0}^{p}-\breve{g}_{p \mu} \Gamma_{\lambda_{0}}^{p}\right) d^{\mu}+\Gamma_{\lambda_{0}}^{i} \breve{g}_{i \mu} d^{\mu}\right) \wedge\left(\breve{g}_{j \nu} d^{\nu}\right) \\
& =c \alpha^{0}\left(-\breve{g}_{i \lambda} d_{0}^{i}+\breve{g}_{i \lambda} \Gamma_{\mu 0}^{i} d^{\mu}\right) \wedge d^{\lambda} .
\end{aligned}
$$

5.4. Proposition. The structures $\left(-c^{2} \tau, \Omega\right)$ and $\left(-\frac{1}{c^{2}} \gamma, \Lambda\right)$ are mutually dual if and only if $\gamma=\gamma[\Gamma]$. 
Proof. Indeed, we have

$$
i_{-c^{2} \tau} \Lambda=-c^{2} i_{\tau} \Lambda=0 \quad \text { and } \quad i_{-\frac{1}{c^{2}} \gamma}\left(-c^{2} \tau\right)=i_{\gamma} \tau=1
$$

Moreover, $i_{-\frac{1}{c^{2}} \gamma} \Omega=0$, if and only if $i_{\gamma} \Omega=0$, i.e. if and only if $\gamma=\gamma[\Gamma]$.

The fact that the maps $\Omega^{b}{ }_{\operatorname{im} \Lambda^{\sharp}}: \operatorname{im} \Lambda^{\sharp} \rightarrow \operatorname{im} \Omega^{b}$ and $\Lambda_{\mid \operatorname{im}}^{\sharp} \Omega^{b}: \operatorname{im} \Omega^{b} \rightarrow \operatorname{im} \Lambda^{\sharp}$ are isomorphisms and

$$
\left(\Omega^{b}{ }_{\operatorname{im} \Lambda^{\sharp}}\right)^{-1}=\Lambda_{\mid \operatorname{im} \Omega^{b}}^{\sharp}, \quad\left(\Lambda_{\mid \operatorname{im} \Omega^{b}}^{\sharp}\right)^{-1}=\Omega_{\mid \operatorname{im} \Lambda^{\sharp}}
$$

follows, by [8], from the fact that dual structures are characterized by

$$
\left(\Lambda^{\sharp} \otimes \Lambda^{\sharp}\right)(\Omega)=-\Lambda, \quad\left(\Omega^{b} \otimes \Omega^{b}\right)(\Lambda)=-\Omega .
$$

Then Proposition follows from Lemma 5.3.

5.5. Remark. Let us remark that dual pairs $\left(-c^{2} \tau, \Omega\right)$ and $\left(-\frac{1}{c^{2}} \gamma, \Lambda\right)$ are characterised by the the following identities [8]

$$
\operatorname{ker}\left(-c^{2} \tau\right)=\operatorname{im} \Lambda^{\sharp}, \quad \operatorname{ker}\left(-\frac{1}{c^{2}} \gamma\right)=\operatorname{im} \Omega^{b} .
$$

5.2. Splittings of the phase tangent and cotangent bundles. Next, we study the splitting of the scaled tangent bundle of the phase space induced by the pair $\left(-\frac{1}{c^{2}} \gamma, \Lambda\right)$ and the splitting of the scaled cotangent bundle of the phase space induced by the pair $\left(-c^{2} \tau, \Omega\right)$.

5.6. Definition. We define the $\gamma$-horizontal and the $\gamma$-vertical subbundles to be, respectively, the vector subbundles

$$
\begin{aligned}
& H_{\gamma} \mathcal{\partial}_{1} \boldsymbol{E}=:\left\langle-\frac{1}{c^{2}} \gamma\right\rangle \subseteq \mathbb{T} \otimes \mathbb{L}^{-2} \otimes T \mathcal{J}_{1} \boldsymbol{E}, \\
& V_{\gamma}^{*} \partial_{1} \boldsymbol{E}=: \operatorname{ker}\left(-\frac{1}{c^{2}} \gamma\right) \subseteq \mathbb{T}^{*} \otimes \mathbb{L}^{2} \otimes T^{*} \mathcal{J}_{1} \boldsymbol{E} .
\end{aligned}
$$

We define the $\tau$-horizontal and the $\tau$-vertical subbundle to be, respectively, the vector subbundles

$$
\begin{aligned}
& H_{\tau}^{*} \mathcal{\partial}_{1} \boldsymbol{E}=:\left\langle-c^{2} \tau\right\rangle \subseteq \mathbb{T}^{*} \otimes \mathbb{L}^{2} \otimes T^{*} \mathcal{\partial}_{1} \boldsymbol{E}, \\
& V_{\tau} \mathcal{\partial}_{1} \boldsymbol{E}=: \operatorname{ker}\left(-c^{2} \tau\right) \subseteq \mathbb{T} \otimes \mathbb{L}^{-2} \otimes T \mathcal{J}_{1} \boldsymbol{E} .
\end{aligned}
$$

5.7. Proposition. We have the linear splittings

$\mathbb{T} \otimes \mathbb{L}^{-2} \otimes T \mathcal{J}_{1} \boldsymbol{E}=H_{\gamma} \mathcal{J}_{1} \boldsymbol{E} \oplus V_{\tau} \mathcal{J}_{1} \boldsymbol{E}=\left\langle-\frac{1}{c^{2}} \gamma\right\rangle \oplus \operatorname{ker}\left(-c^{2} \tau\right)=\left\langle-\frac{1}{c^{2}} \gamma\right\rangle \oplus \operatorname{im} \Lambda^{\sharp}$,

$\mathbb{T}^{*} \otimes \mathbb{L}^{2} \otimes T^{*} \mathcal{J}_{1} \boldsymbol{E}=H_{\tau}^{*} \mathcal{J}_{1} \boldsymbol{E} \oplus V_{\gamma}^{*} \mathcal{J}_{1} \boldsymbol{E}=\left\langle-c^{2} \tau\right\rangle \oplus \operatorname{ker}\left(-\frac{1}{c^{2}} \gamma\right)=\left\langle-c^{2} \tau\right\rangle \oplus \operatorname{im} \Omega^{b}$.

We have the mutually dual local bases of phase vector fields $\left(e_{0}, e_{i}, e_{i}^{0}\right)$ and of phase 1 -forms $\left(\epsilon^{0}, \epsilon^{i}, \epsilon_{0}^{i}\right)$ adapted to the above splittings, where

$$
e_{0}=: \breve{\delta}^{\lambda}\left(\partial_{\lambda}+\Gamma_{\lambda_{0}}^{i} \partial_{i}^{0}\right), \quad e_{i}=:\left(\delta_{i}^{\lambda}+\left(\alpha^{0}\right)^{2} \breve{g}_{0 i} \breve{\delta}_{0}^{\lambda}\right)\left(\partial_{\lambda}+\Gamma_{\lambda_{0}}^{i} \partial_{i}^{0}\right), \quad e_{i}^{0}=: \partial_{i}^{0},
$$

and

$$
\epsilon^{0}=:-\left(\alpha^{0}\right)^{2} \breve{g}_{0 \lambda} d^{\lambda}, \quad \epsilon^{i}=: d^{i}-x_{0}^{i} d^{0}, \quad \epsilon_{0}^{i}=: d_{0}^{i}-\Gamma_{\lambda_{0}}^{i} d^{\lambda} .
$$


We have the inverse relations

$$
\partial_{\lambda}=-\left(\alpha^{0}\right)^{2} \breve{g}_{0 \lambda} e_{0}+\breve{\delta}_{\lambda}^{i} e_{i}-\Gamma_{\lambda_{0}}^{i} e_{i}^{0}, \quad \partial_{i}^{0}=e_{i}^{0} .
$$

and

$$
\begin{aligned}
& d^{0}=\epsilon^{0}+\left(\alpha^{0}\right)^{2} \breve{g}_{0 i} \epsilon^{i}, \quad d^{i}=\epsilon^{i}+x_{0}^{i}\left(\epsilon^{0}+\left(\alpha^{0}\right)^{2} \breve{g}_{0 j} \epsilon^{j}\right), \\
& d_{0}^{i}=\epsilon_{0}^{i}+\left(\Gamma_{j 0}^{i}+\left(\alpha^{0}\right)^{2} \breve{g}_{0 j} \breve{\delta}_{0}^{\rho} \Gamma_{\rho 0}^{i}\right) \epsilon^{j}+\breve{\delta}_{0}^{\rho} \Gamma_{\rho 0}^{i} \epsilon^{0} .
\end{aligned}
$$

5.8. Proposition. The projection $\mathbb{T} \otimes \mathbb{L}^{-2} \otimes T \mathcal{J}_{1} \boldsymbol{E} \rightarrow H_{\gamma} \mathcal{J}_{1} \boldsymbol{E}$ is given by

$$
X \mapsto\left(-c^{2} \tau\right)(X)\left(-\frac{1}{c^{2}} \gamma\right)=\tau(X) \gamma
$$

and the projection $\mathbb{T} \otimes \mathbb{L}^{-2} \otimes T \mathcal{J}_{1} \boldsymbol{E} \rightarrow V_{\tau} \mathcal{\partial}_{1} \boldsymbol{E}$ is given by

$$
X \mapsto(X-\tau(X) \gamma),
$$

for each $X \in \mathbb{T} \otimes \mathbb{L}^{-2} \otimes T \mathcal{J}_{1} \boldsymbol{E}$.

The projection $\mathbb{T}^{*} \otimes \mathbb{L}^{2} \otimes T^{*} \mathcal{J}_{1} \boldsymbol{E} \rightarrow H_{\tau}^{*} \mathcal{J}_{1} \boldsymbol{E}$ is given by

$$
\phi \mapsto\left(-\frac{1}{c^{2}} \gamma\right)(\phi)\left(-c^{2} \tau\right)=\gamma(\phi) \tau
$$

and the projection $\mathbb{T}^{*} \otimes \mathbb{L}^{2} \otimes T^{*} \mathcal{J}_{1} \boldsymbol{E} \rightarrow V_{\gamma}^{*} \mathcal{J}_{1} \boldsymbol{E}$ is given by

$$
\phi \mapsto \phi-\gamma(\phi) \tau,
$$

for each $\phi \in \mathbb{T}^{*} \otimes \mathbb{L}^{2} \otimes T^{*} \mathcal{J}_{1} \boldsymbol{E}$.

5.9. Corollary. Each $X \in \sec \left(\mathcal{J}_{1} \boldsymbol{E}, \mathbb{T} \otimes \mathbb{L}^{-2} \otimes T \mathcal{J}_{1} \boldsymbol{E}\right)$ can be uniquely split as

$$
X=\tau(X) \gamma+(X-\tau(X) \gamma) \text {. }
$$

If the coordinate expression of $X$ in a spacetime chart is $X=X^{0 \lambda} u_{0} \otimes \partial_{\lambda}+X_{0}^{0 i} u_{0} \otimes \partial_{i}^{0}$, with $X^{0 \lambda}, X_{0}^{0 i} \in \operatorname{map}\left(\mathcal{J}_{1} \boldsymbol{E}, \mathbb{L}^{-2} \otimes \mathbb{R}\right)$, then the expression of its splitting in the adapted base (5.1) is

$$
X=-\left(\alpha^{0}\right)^{2} \breve{g}_{0 \lambda} X^{0 \lambda} u_{0} \otimes e_{0}+\breve{\delta}_{\lambda}^{i} X^{0 \lambda} u_{0} \otimes e_{i}+\left(X_{0}^{0 i}-\Gamma_{\lambda_{0}}^{i} X^{0 \lambda}\right) u_{0} \otimes e_{i}^{0} .
$$

Each $\phi \in \sec \left(\mathcal{J}_{1} \boldsymbol{E}, \mathbb{T}^{*} \otimes \mathbb{L}^{2} \otimes T^{*} \mathcal{J}_{1} \boldsymbol{E}\right)$ can be uniquely split as

$$
\phi=\gamma(\phi) \tau+(\phi-\gamma(\phi) \tau) \text {. }
$$

If the coordinate expression of $\phi$ in a spacetime chart is $\phi=\phi_{0 \lambda} u^{0} \otimes d^{\lambda}+\phi_{0 i}^{0} u^{0} \otimes d_{0}^{i}$, with $\phi_{0 \lambda}, \phi_{0 i}^{0} \in \operatorname{map}\left(\mathcal{J}_{1} \boldsymbol{E}, \mathbb{L}^{2} \otimes \mathbb{R}\right)$, then the expression of its splitting in the adapted base (5.2) is

$$
\begin{aligned}
\phi= & \breve{\delta}_{0}^{\rho}\left(\phi_{0 \rho}+\phi_{0 p}^{0} \Gamma_{\rho 0}^{p}\right) u^{0} \otimes \epsilon^{0} \\
& +\left(\phi_{0 i}+\phi_{0 p}^{0} \Gamma_{i 0}^{p}+\left(\alpha^{0}\right)^{2} \breve{g}_{0 i} \breve{\delta}_{0}^{\rho}\left(\phi_{0 \rho}+\phi_{0 p}^{0} \Gamma_{\rho 0}^{p}\right)\right) u^{0} \otimes \epsilon^{i}+\phi_{0 i}^{0} u_{0} \otimes \epsilon_{0}^{i} .
\end{aligned}
$$

5.10. Corollary. If the coordinate expression of $X$ in a spacetime chart and in a adapted base are

$$
\begin{aligned}
X & =X^{0 \lambda} u_{0} \otimes \partial_{\lambda}+X_{0}^{0 i} u_{0} \otimes \partial_{i}^{0}, \\
& =\widetilde{X}^{00} u_{0} \otimes e_{0}+\widetilde{X}^{0 i} u_{0} \otimes e_{i}+\widetilde{X}_{0}^{0 i} u_{0} \otimes e_{i}^{0},
\end{aligned}
$$


with $X^{0 \lambda}, X_{0}^{0 i}, \widetilde{X}^{00}, \widetilde{X}^{0 i}, \widetilde{X}_{0}^{0 i} \in \operatorname{map}\left(\mathcal{J}_{1} \boldsymbol{E}, \mathbb{L}^{-2} \otimes \mathbb{R}\right)$, then we have the equalities

$$
\begin{aligned}
& \widetilde{X}^{00}=-\left(\alpha^{0}\right)^{2} \breve{g}_{0 \lambda} X^{0 \lambda}, \quad \widetilde{X}^{0 i}=\breve{\delta}_{\lambda}^{i} X^{0 \lambda}, \quad \widetilde{X}_{0}^{0 i}=X_{0}^{0 i}-\Gamma_{\lambda_{0}}^{i} X^{0 \lambda}, \\
& X^{00}=\widetilde{X}^{00}+\left(\alpha^{0}\right)^{2} \breve{g}_{0 p} \widetilde{X}^{0 p}, \quad X^{0 i}=\widetilde{X}^{0 i}+x_{0}^{i}\left(\widetilde{x}^{00}+\left(\alpha^{0}\right)^{2} \breve{g}_{0 p} \widetilde{X}^{0 p},\right. \\
& X_{0}^{0 i}=\widetilde{X}_{0}^{0 i}+\left(\Gamma_{p 0}^{i}+\left(\alpha^{0}\right)^{2} \breve{g}_{0 p} \breve{\delta}_{0}^{\rho} \Gamma_{\rho 0}^{i}\right) \widetilde{X}^{0 p}+\breve{\delta}_{0}^{\rho} \Gamma_{\rho 0}^{i} \widetilde{X}^{00} .
\end{aligned}
$$

If the coordinate expression of $\phi$ in a spacetime chart and in a adapted base are

$$
\begin{aligned}
\phi & =\phi_{0 \lambda} u^{0} \otimes d^{\lambda}+\phi_{0 i}^{0} u^{0} \otimes d_{0}^{i} \\
& =\widetilde{\phi}_{00} u^{0} \otimes \epsilon^{0}+\widetilde{\phi}_{0 i} u^{0} \otimes \epsilon^{i}+\widetilde{\phi}_{0 i}^{0} u^{0} \otimes \epsilon_{0}^{i},
\end{aligned}
$$

with $\phi_{0 \lambda}, \phi_{0 i}^{0}, \widetilde{\phi}_{00}, \widetilde{\phi}_{0 i}, \widetilde{\phi}_{0 i}^{0} \in \operatorname{map}\left(\mathcal{J}_{1} \boldsymbol{E}, \mathbb{L}^{2} \otimes \mathbb{R}\right)$, then we have the equalities

$$
\begin{aligned}
& \widetilde{\phi}_{00}=\breve{\delta}_{0}^{\lambda}\left(\phi_{0 \lambda}+\phi_{0 p}^{0} \Gamma_{\lambda 0}^{p}\right), \quad \widetilde{\phi}_{0 i}=\phi_{0 i}+\phi_{0 p}^{0} \Gamma_{i 0}^{p}+\left(\alpha^{0}\right)^{2} \breve{g}_{0 i} \breve{\delta}_{0}^{\lambda}\left(\phi_{0 \lambda}+\phi_{0 p}^{0} \Gamma_{\lambda_{0}}^{p}\right), \\
& \widetilde{\phi}_{0 i}^{0}=\phi_{0 i}^{0}, \\
& \phi_{0 \lambda}=-\left(\alpha^{0}\right)^{2} \breve{g}_{0 \lambda} \widetilde{\phi}_{00}-\breve{\delta}_{\lambda}^{p} \widetilde{\phi}_{0 p}-\widetilde{\phi}_{0 p}^{0} \Gamma_{\lambda 0}^{p}, \quad \phi_{0 i}^{0}=\widetilde{\phi}_{0 i}^{0} .
\end{aligned}
$$

5.11. Proposition. The musical morphisms $\Lambda^{\sharp}: T^{*} \mathcal{J}_{1} \boldsymbol{E} \rightarrow \mathbb{T} \otimes \mathbb{L}^{-2} \otimes T \mathcal{J}_{1} \boldsymbol{E}$ and $\Omega^{b}: T \mathcal{J}_{1} \boldsymbol{E} \rightarrow \mathbb{T}^{*} \otimes \mathbb{L}^{2} \otimes T^{*} \mathcal{J}_{1} \boldsymbol{E}$ can be naturally identified, respectively, with the morphisms $\Lambda^{\sharp}: \mathbb{T}^{*} \otimes \mathbb{L}^{2} \otimes T^{*} \mathcal{J}_{1} \boldsymbol{E} \rightarrow T \mathcal{J}_{1} \boldsymbol{E}$ and $\Omega^{b}: \mathbb{T} \otimes \mathbb{L}^{-2} \otimes T \mathcal{J}_{1} \boldsymbol{E} \rightarrow T^{*} \mathcal{J}_{1} \boldsymbol{E}$.

Moreover, these morphisms can be naturally extended, respectively, to the morphisms, denoted by the same symbols, $\Lambda^{\sharp}: \mathbb{T}^{*} \otimes \mathbb{L}^{2} \otimes T^{*} \mathcal{J}_{1} \boldsymbol{E} \rightarrow \mathbb{T} \otimes \mathbb{L}^{-2} \otimes T \mathcal{J}_{1} \boldsymbol{E}$ and $\Omega^{b}: \mathbb{T} \otimes \mathbb{L}^{-2} \otimes T \mathcal{J}_{1} \boldsymbol{E} \rightarrow \mathbb{T}^{*} \otimes \mathbb{L}^{2} \otimes T^{*} \mathcal{J}_{1} \boldsymbol{E}$.

Furthermore, the restrictions $\Lambda^{\sharp}: V_{\gamma}^{*} \mathcal{J}_{1} \boldsymbol{E} \rightarrow V_{\tau} \mathcal{\partial}_{1} \boldsymbol{E}$ and $\Omega^{b}: V_{\tau} \mathcal{\partial}_{1} \boldsymbol{E} \rightarrow V_{\gamma}^{*} \mathcal{J}_{1} \boldsymbol{E}$ are mutually inverse isomorphisms.

5.12. Proposition. We have the morphisms

$$
\begin{aligned}
& \gamma^{\sharp}: \mathbb{T}^{*} \otimes \mathbb{L}^{2} \otimes T^{*} \mathcal{J}_{1} \boldsymbol{E} \rightarrow \mathbb{T} \otimes \mathbb{L}^{-2} \otimes T \mathcal{J}_{1} \boldsymbol{E}: \phi \mapsto \phi\left(-\frac{1}{c^{2}} \gamma\right)\left(-\frac{1}{c^{2}} \gamma\right), \\
& \tau^{b}: \mathbb{T} \otimes \mathbb{L}^{-2} \otimes T \mathcal{J}_{1} \boldsymbol{E} \rightarrow \mathbb{T}^{*} \otimes \mathbb{L}^{2} \otimes T^{*} \mathcal{J}_{1} \boldsymbol{E}: X \mapsto\left(-c^{2} \tau\right)(X)\left(-c^{2} \tau\right) .
\end{aligned}
$$

Moreover, the restrictions $\gamma^{\sharp}: H_{\tau}^{*} \mathcal{J}_{1} \boldsymbol{E} \rightarrow H_{\gamma} \mathcal{\partial}_{1} \boldsymbol{E}$ and $\tau^{b}: H_{\gamma} \mathcal{\partial}_{1} \boldsymbol{E} \rightarrow H_{\tau}^{*} \mathcal{J}_{1} \boldsymbol{E}$ are mutually inverse isomorphisms given by $f\left(-c^{2} \tau\right) \mapsto f\left(-\frac{1}{c^{2}} \gamma\right)$ and $f\left(-\frac{1}{c^{2}} \gamma\right) \mapsto f\left(-c^{2} \tau\right)$, respectively, with $f \in \operatorname{map}\left(\mathcal{J}_{1} \boldsymbol{E}, \mathbb{R}\right)$.

5.13. Note. By considering the Planck constant $\hbar$ and a particle of mass $m$, we define also the rescaled morphisms, denoted by the same symbol,

$$
\begin{gathered}
\gamma^{\sharp}: T^{*} \mathcal{J}_{1} \boldsymbol{E} \rightarrow \mathbb{T} \otimes \mathbb{L}^{-2} \otimes T \mathcal{J}_{1} \boldsymbol{E}: \varphi \mapsto \frac{\hbar}{m c^{4}} \gamma(\varphi) \gamma, \\
\tau^{b}: T \mathcal{J}_{1} \boldsymbol{E} \rightarrow \mathbb{T}^{*} \otimes \mathbb{L}^{2} \otimes T^{*} \mathcal{J}_{1} \boldsymbol{E}: Y \mapsto \frac{m c^{4}}{\hbar} \tau(Y) \tau .
\end{gathered}
$$

5.14. Proposition. The phase tangent and cotangent splittings and the isomorphisms $\gamma^{\sharp}\left|H_{\tau}^{* \mathcal{J}_{1} \boldsymbol{E}}, \Lambda^{\sharp}\right| V_{\gamma}^{*} \mathcal{J}_{1} \boldsymbol{E}, \tau^{b}{ }_{\mid H_{\gamma} \mathcal{J}_{1} \boldsymbol{E}}$ and $\Omega^{b}{ }_{\mid V_{\tau} \mathcal{J}_{1} \boldsymbol{E}}$ define the mutually inverse isomorphisms

$$
\begin{aligned}
& { }^{\sharp}: \mathbb{T}^{*} \otimes \mathbb{L}^{2} \otimes T^{*} \mathcal{J}_{1} \boldsymbol{E} \rightarrow \mathbb{T} \otimes \mathbb{L}^{-2} \otimes T \mathcal{J}_{1} \boldsymbol{E}, \\
& \text { ь }: \mathbb{T} \otimes \mathbb{L}^{-2} \otimes T \mathcal{J}_{1} \boldsymbol{E} \rightarrow \mathbb{T}^{*} \otimes \mathbb{L}^{2} \otimes T^{*} \mathcal{J}_{1} \boldsymbol{E},
\end{aligned}
$$


hence, the mutually inverse rescaled isomorphisms

$$
\begin{aligned}
& { }^{\sharp}: T^{*} \mathcal{J}_{1} \boldsymbol{E} \rightarrow \mathbb{T} \otimes \mathbb{L}^{-2} \otimes T \mathcal{J}_{1} \boldsymbol{E}, \\
& { }^{\text {ь }}: \mathbb{T} \otimes \mathbb{L}^{-2} \otimes T \mathcal{J}_{1} \boldsymbol{E} \rightarrow T^{*} \mathcal{g}_{1} \boldsymbol{E}, \\
& { }^{\sharp}: \mathbb{T}^{*} \otimes \mathbb{L}^{2} \otimes T^{*} \mathcal{J}_{1} \boldsymbol{E} \rightarrow T \mathcal{J}_{1} \boldsymbol{E}, \\
& { }^{\text {ь }}: T \mathcal{J}_{1} \boldsymbol{E} \rightarrow \mathbb{T}^{*} \otimes \mathbb{L}^{2} \otimes T^{*} \mathcal{J}_{1} \boldsymbol{E} .
\end{aligned}
$$

5.15. Corollary. In the adapted bases (5.1) and (5.2) we have

$$
\begin{aligned}
\sharp\left(\epsilon^{0}\right)=: \frac{\hbar}{m c^{4}} \gamma\left(\epsilon^{0}\right) \gamma & =\frac{\hbar\left(\alpha^{0}\right)^{2}}{m c^{2}} e_{0}, \quad{ }^{\sharp}\left(\epsilon^{i}\right)=: i_{\epsilon^{i}} \Lambda=\frac{1}{c \alpha^{0}} \hat{g}^{i j} e_{j}^{0}, \\
\sharp\left(\epsilon_{0}^{i}\right) & =: i_{\epsilon_{0}^{i}} \Lambda=-\frac{1}{c \alpha^{0}} \hat{g}^{i j} e_{j},
\end{aligned}
$$

and

$$
\begin{gathered}
{ }^{b}\left(e_{0}\right)=: \frac{m c^{4}}{\hbar} \tau\left(e_{0}\right) \tau=\frac{m c^{2}}{\hbar\left(\alpha^{0}\right)^{2}} \epsilon^{0}, \quad{ }^{b}\left(e_{i}\right)=: i_{e_{i}} \Omega=-c \alpha^{0} \hat{g}_{i j} \epsilon_{j}^{0}, \\
{ }^{b}\left(e_{i}^{0}\right)=: i_{e_{i}^{0}} \Omega=c \alpha^{0} \hat{g}_{i j} \epsilon^{j} .
\end{gathered}
$$

Thus, if $\varphi \in \sec \left(\mathcal{J}_{1} \boldsymbol{E}, T^{*} \mathcal{J}_{1} \boldsymbol{E}\right)$ and $Y \in \sec \left(\mathcal{J}_{1} \boldsymbol{E}, T^{*} \mathcal{J}_{1} \boldsymbol{E}\right)$ have the coordinate expressions $\varphi=\widetilde{\varphi}_{\lambda} \epsilon^{\lambda}+\widetilde{\varphi}_{i}^{0} \epsilon_{0}^{i}$ and $Y=\widetilde{Y}^{\lambda} e_{\lambda}+\widetilde{Y}_{0}^{i} e_{i}^{0}$, with $\widetilde{\varphi}_{\lambda}, \widetilde{\varphi}_{i}^{0}, \widetilde{Y}^{\lambda}, \widetilde{Y}_{0}^{i} \in \operatorname{map}\left(\mathcal{J}_{1} \boldsymbol{E}, \mathbb{R}\right)$, then

$$
\begin{aligned}
& \sharp(\varphi)=\frac{\hbar\left(\alpha^{0}\right)^{2}}{m c^{2}} \widetilde{\varphi}_{0} e_{0}-\frac{1}{c \alpha^{0}} \hat{g}^{i j} \widetilde{\varphi}_{j}^{0} e_{i}+\frac{1}{c \alpha^{0}} \hat{g}^{i j} \widetilde{\varphi}_{j} e_{i}^{0} \\
& { }^{\mathrm{b}}(Y)=\frac{m c^{2}}{\hbar\left(\alpha^{0}\right)^{2}} \widetilde{Y}^{0} \epsilon^{0}-c \alpha^{0} \hat{g}_{i j} \widetilde{Y}_{0}^{j} \epsilon^{i}+c \alpha^{0} \hat{g}_{i j} \widetilde{Y}^{j} \epsilon_{0}^{i} .
\end{aligned}
$$

5.3. Almost-cosymplectic-contact structure. Then, we study the conditions by which $\Omega$ is closed.

5.16. Lemma. The scaled phase $2-$ form $\Omega$ is closed if and only if the following conditions are satisfied

$$
\begin{aligned}
\partial_{\lambda}\left(\alpha^{0} \breve{g}_{i \mu}\right)+ & \partial_{i}^{0}\left(\alpha^{0} \breve{g}_{j \mu} \Gamma_{\lambda 0}^{j}\right)-\partial_{\mu}\left(\alpha^{0} \breve{g}_{i \lambda}\right)-\partial_{i}^{0}\left(\alpha^{0} \breve{g}_{j \lambda} \Gamma_{\mu 0}^{j}\right)=0, \\
& \breve{g}_{j \mu} R_{\nu \lambda_{0}}^{j}+\breve{g}_{j \nu} R_{\lambda \mu 0}^{j}+\breve{g}_{j \lambda} R_{\mu \nu 0}^{j}=0 .
\end{aligned}
$$

Proof. From the coordinate expression of $\Omega$ we obtain

$$
\begin{aligned}
d \Omega= & c \partial_{i}^{0}\left(\alpha^{0} \breve{g}_{j \mu}\right) d_{0}^{i} \wedge d_{0}^{j} \wedge d^{\mu}-c\left(\partial_{\lambda}\left(\alpha^{0} \breve{g}_{i \mu}\right)+\partial_{i}^{0}\left(\alpha^{0} \breve{g}_{j \mu} \Gamma_{\lambda_{0}}^{j}\right)\right) d_{0}^{i} \wedge d^{\lambda} \wedge d^{\mu} \\
& -c \partial_{\nu}\left(\alpha^{0} \breve{g}_{j \mu} \Gamma_{\lambda_{0}}^{j}\right) d^{\nu} \wedge d^{\lambda} \wedge d^{\mu} .
\end{aligned}
$$

Hence, $d \Omega=0$ if and only if

$$
\begin{array}{r}
\partial_{i}^{0}\left(\alpha^{0} \breve{g}_{j \mu}\right)-\partial_{j}^{0}\left(\alpha^{0} \breve{g}_{i \mu}\right)=0, \\
\partial_{\lambda}\left(\alpha^{0} \breve{g}_{i \mu}\right)+\partial_{i}^{0}\left(\alpha^{0} \breve{g}_{j \mu} \Gamma_{\lambda 0}^{j}\right)-\partial_{\mu}\left(\alpha^{0} \breve{g}_{i \lambda}\right)-\partial_{i}^{0}\left(\alpha^{0} \breve{g}_{j \lambda} \Gamma_{\mu 0}^{j}\right)=0, \\
\partial_{\lambda}\left(\alpha^{0} \breve{g}_{j \mu} \Gamma_{\nu 0}^{j}\right)-\partial_{\mu}\left(\alpha^{0} \breve{g}_{j \lambda} \Gamma_{\nu 0}^{j}\right)+\partial_{\nu}\left(\alpha^{0} \breve{g}_{j \lambda} \Gamma_{\mu_{0}}^{j}\right)-\partial_{\lambda}\left(\alpha^{0} \breve{g}_{j \nu} \Gamma_{\mu 0}^{j}\right)+
\end{array}
$$




$$
+\partial_{\mu}\left(\alpha^{0} \breve{g}_{j \nu} \Gamma_{\lambda_{0}}^{j}\right)-\partial_{\nu}\left(\alpha^{0} \breve{g}_{j \mu} \Gamma_{\lambda_{0}}^{j}\right)=0
$$

We have

$$
\partial_{i}^{0}\left(\alpha^{0} \breve{g}_{j \mu}\right)=\left(\alpha^{0}\right)^{3}\left(\breve{g}_{0 i} \breve{g}_{j \mu}+\breve{g}_{0 j} \breve{g}_{i \mu}+\hat{g}_{i j} \breve{g}_{0 \mu}\right) .
$$

Hence, $\partial_{i}^{0}\left(\alpha^{0} \breve{g}_{j \mu}\right)$ is symmetric with respect to $i, j$, which implies that (5.8) is satisfied identically.

On the other hand, we can rewrite (5.10) as

$$
\begin{aligned}
\alpha^{0} \breve{g}_{j \mu}\left(\partial_{\lambda} \Gamma_{\nu 0}^{j}-\partial_{\nu} \Gamma_{\lambda 0}^{j}\right)+\alpha^{0} \breve{g}_{j \nu}\left(\partial_{\mu} \Gamma_{\lambda 0}^{j}-\partial_{\lambda} \Gamma_{\mu 0}^{j}\right)+\alpha^{0} \breve{g}_{j \lambda}\left(\partial_{\nu} \Gamma_{\mu 0}^{j}-\partial_{\mu} \Gamma_{\nu 0}^{j}\right)+ & \\
+\left(\partial_{\lambda}\left(\alpha^{0} \breve{g}_{j \mu}\right)-\partial_{\mu}\left(\alpha^{0} \breve{g}_{j \lambda}\right)\right) \Gamma_{\nu 0}^{j}+ & \left(\partial_{\nu}\left(\alpha^{0} \breve{g}_{j \lambda}\right)-\partial_{\lambda}\left(\alpha^{0} \breve{g}_{j \nu}\right)\right) \Gamma_{\mu 0}^{j}+ \\
& +\left(\partial_{\mu}\left(\alpha^{0} \breve{g}_{j \nu}\right)-\partial_{\nu}\left(\alpha^{0} \breve{g}_{j \mu}\right)\right) \Gamma_{\lambda_{0}}^{j}=0
\end{aligned}
$$

and $(5.9)$ as

$$
\partial_{\lambda}\left(\alpha^{0} \breve{g}_{i \mu}\right)-\partial_{\mu}\left(\alpha^{0} \breve{g}_{i \lambda}\right)=\partial_{i}^{0}\left(\alpha^{0} \breve{g}_{j \lambda} \Gamma_{\mu 0}^{j}\right)-\partial_{i}^{0}\left(\alpha^{0} \breve{g}_{j \mu} \Gamma_{\lambda_{0}}^{j}\right)
$$

Then, by inserting (5.12) and its permutations into (5.11), we get

$$
\begin{array}{r}
\left(\partial_{h}^{0}\left(\alpha^{0} \breve{g}_{j \lambda} \Gamma_{\mu 0}^{j}\right)-\partial_{h}^{0}\left(\alpha^{0} \breve{g}_{j \mu} \Gamma_{\lambda 0}^{j}\right)\right) \Gamma_{\nu 0}^{h}+\left(\partial_{h}^{0}\left(\alpha^{0} \breve{g}_{j \nu} \Gamma_{\lambda 0}^{j}\right)-\partial_{h}^{0}\left(\alpha^{0} \breve{g}_{j \lambda} \Gamma_{\nu 0}^{j}\right)\right) \Gamma_{\mu 0}^{h}+ \\
+\left(\partial_{h}^{0}\left(\alpha^{0} \breve{g}_{j \mu} \Gamma_{\nu 0}^{j}\right)-\partial_{h}^{0}\left(\alpha^{0} \breve{g}_{j \nu} \Gamma_{\mu 0}^{j}\right)\right) \Gamma_{\lambda_{0}}^{h}+\alpha^{0} \breve{g}_{j \mu}\left(\partial_{\lambda} \Gamma_{\nu 0}^{j}-\partial_{\nu} \Gamma_{\lambda 0}^{j}\right)+ \\
+\alpha^{0} \breve{g}_{j \nu}\left(\partial_{\mu} \Gamma_{\lambda_{0}}^{j}-\partial_{\lambda} \Gamma_{\mu 0}^{j}\right)+\alpha^{0} \breve{g}_{j \lambda}\left(\partial_{\nu} \Gamma_{\mu 0}^{j}-\partial_{\mu} \Gamma_{\nu 0}^{j}\right)= \\
\alpha^{0} \breve{g}_{j \mu}\left(\partial_{\lambda} \Gamma_{\nu 0}^{j}-\partial_{\nu} \Gamma_{\lambda_{0}}^{j}+\Gamma_{\lambda 0}^{h} \partial_{h}^{0} \Gamma_{\nu 0}^{j}-\Gamma_{\nu 0}^{h} \partial_{h}^{0} \Gamma_{\lambda 0}^{j}\right)+ \\
+\alpha^{0} \breve{g}_{j \nu}\left(\partial_{\mu} \Gamma_{\lambda 0}^{j}-\partial_{\lambda} \Gamma_{\mu 0}^{j}+\Gamma_{\mu 0}^{h} \partial_{h}^{0} \Gamma_{\lambda 0}^{j}-\Gamma_{\lambda 0}^{h} \partial_{h}^{0} \Gamma_{\mu 0}^{j}\right)+ \\
+\alpha^{0} \breve{g}_{j \lambda}\left(\partial_{\nu} \Gamma_{\mu 0}^{j}-\partial_{\mu} \Gamma_{\nu 0}^{j}+\Gamma_{\nu 0}^{h} \partial_{h}^{0} \Gamma_{\mu 0}^{j}-\Gamma_{\mu 0}^{h} \partial_{h}^{0} \Gamma_{\nu 0}^{j}\right)+ \\
+\left(\Gamma_{\lambda 0}^{h} \Gamma_{\kappa 0}^{j}-\Gamma_{\kappa 0}^{h} \Gamma_{\lambda 0}^{j}\right) \partial_{q}^{0}\left(\alpha^{0} \breve{g}_{j \mu}\right)+\left(\Gamma_{\mu 0}^{h} \Gamma_{\lambda 0}^{j}-\Gamma_{\lambda_{0}}^{h} \Gamma_{\mu 0}^{j}\right) \partial_{h}^{0}\left(\alpha^{0} \breve{g}_{j \nu}\right)+ \\
+\left(\Gamma_{\nu 0}^{h} \Gamma_{\mu 0}^{j}-\Gamma_{\mu 0}^{h} \Gamma_{\nu 0}^{j}\right) \partial_{h}^{0}\left(\alpha^{0} \breve{g}_{j \lambda}\right)= \\
\alpha^{0}\left(\breve{g}_{j \mu} R_{\nu \lambda_{0}}^{j}+\breve{g}_{j \nu} R_{\lambda \mu 0}^{j}+\breve{g}_{j \lambda} R_{\mu \nu 0}^{j}\right)=0 .
\end{array}
$$

Hence, (5.9) and (5.10) are equivalent to (5.5) and (5.6).

5.17. Note. By using the above computations we obtain the coordinate expression

$$
\begin{aligned}
d \Omega= & \frac{1}{2} c \alpha^{0} \breve{g}_{j \mu} R_{\nu \lambda 0}^{j} d^{\nu} \wedge d^{\lambda} \wedge d^{\mu}-c\left(\alpha^{0} \breve{g}_{j \mu} \partial_{i}^{0} \Gamma_{\lambda 0}^{j}+\partial_{\lambda}\left(\alpha^{0} \breve{g}_{i \mu}\right)\right)\left(d_{0}^{i}-\Gamma_{\nu 0}^{i} d^{\nu}\right) \wedge d^{\lambda} \wedge d^{\mu} \\
& +c \partial_{i}^{0}\left(\alpha^{0} \breve{g}_{j \mu}\right) d_{0}^{i} \wedge\left(d_{0}^{j}-\Gamma_{\lambda 0}^{j} d^{\lambda}\right) \wedge d^{\mu}
\end{aligned}
$$

Now, we provide a geometric interpretation of identities (5.5) and (5.6). For this purpose, let us note that we can define the Lie derivatives of $\tau$ with respect to $\Gamma$ and $R$ according to (2.1).

5.18. Proposition. The scaled phase $2-$ form $\Omega$ is closed if and only if

$$
L_{\nu_{\tau}(X)} L_{\Gamma} \tau=0, \quad \forall X \in \sec (\boldsymbol{E}, T \boldsymbol{E}), \quad \text { and } \quad L_{R} \tau=0 .
$$


Proof. The sections

$$
L_{\Gamma} \tau: \partial_{1} \boldsymbol{E} \rightarrow \mathbb{T} \otimes \Lambda^{2} T^{*} \boldsymbol{E} \quad \text { and } \quad L_{R} \tau: \partial_{1} \boldsymbol{E} \rightarrow \mathbb{T} \otimes \Lambda^{3} T^{*} \boldsymbol{E}
$$

have the coordinate expressions

$$
\begin{aligned}
L_{\Gamma} \tau & =\left(\partial_{\lambda} \tau_{\mu}+\Gamma_{\lambda_{0}}^{j} \partial_{j}^{0} \tau_{\mu}\right) d^{\lambda} \wedge d^{\mu}=-\frac{1}{c}\left(\partial_{\lambda}\left(\alpha^{0} \breve{g}_{0 \mu}\right)+\Gamma_{\lambda_{0}}^{j} \partial_{j}^{0}\left(\alpha^{0} \breve{g}_{0 \mu}\right)\right) d^{\lambda} \wedge d^{\mu} \\
& =-\frac{1}{c}\left(\partial_{\lambda}\left(\alpha^{0} \breve{g}_{0 \mu}\right)+\alpha^{0} \breve{g}_{j \mu} \Gamma_{\lambda_{0}}^{j}\right) d^{\lambda} \wedge d^{\mu} \\
& =\frac{\alpha^{0}}{c}\left(\partial_{\mu} \breve{g}_{0 \lambda}-g_{j \mu} \Gamma_{\lambda_{0}}^{j}+\left(\alpha^{0}\right)^{2} \breve{g}_{0 \lambda}\left(\frac{1}{2} \partial_{\mu} \hat{g}_{00}+\breve{g}_{0 p} \Gamma_{\mu 0}^{p}\right)\right) d^{\lambda} \wedge d^{\mu}
\end{aligned}
$$

and

$$
L_{R} \tau=-\frac{\alpha^{0}}{c} \breve{g}_{i \lambda} R_{\mu \nu 0}^{i} d^{\lambda} \wedge d^{\mu} \wedge d^{\nu}=-\frac{\alpha^{0}}{c} \hat{g}_{i j} R_{\mu \nu 0}^{i} \beta^{j} \wedge d^{\mu} \wedge d^{\nu} .
$$

Hence, $L_{R} \tau=0$ if and only if the identity (5.6) is satisfied.

Then, for each $X=X^{\lambda} \partial_{\lambda}$, we have the coordinate expression

$$
\begin{aligned}
L_{\nu_{\tau}(X)} L_{\Gamma} \tau & =\frac{1}{c \alpha^{0}}\left[\partial_{i}^{0} \partial_{\lambda} \tau_{\mu}+\partial_{i}^{0}\left(\Gamma_{\lambda_{0}}^{j} \partial_{j}^{0} \tau_{\mu}\right)\right] \tilde{X}^{i} d^{\lambda} \wedge d^{\mu} \\
& =-\frac{1}{c^{2} \alpha^{0}}\left[\partial_{\lambda} \partial_{i}^{0}\left(\alpha^{0} \breve{g}_{0 \mu}\right)+\partial_{i}^{0}\left(\Gamma_{\lambda_{0}}^{j} \partial_{j}^{0}\left(\alpha^{0} \breve{g}_{0 \mu}\right)\right)\right] \tilde{X}^{i} d^{\lambda} \wedge d^{\mu},
\end{aligned}
$$

where $\tilde{X}^{i}=X^{i}-x_{0}^{i} X^{0}$.

Moreover, we have

$$
\partial_{\lambda} \partial_{i}^{0}\left(\alpha^{0} \breve{g}_{0 \mu}\right)+\partial_{i}^{0}\left(\Gamma_{\lambda 0}^{j} \partial_{p}^{0}\left(\alpha^{0} \breve{g}_{0 \mu}\right)\right)=\partial_{\lambda}\left(\alpha^{0} \breve{g}_{i \mu}\right)+\partial_{i}^{0}\left(\alpha^{0} \breve{g}_{j \mu} \Gamma_{\lambda 0}^{j}\right)
$$

which implies

$$
L_{\nu_{\tau}(X)} L_{\Gamma} \tau=-\frac{1}{c^{2} \alpha^{0}}\left[\partial_{\lambda}\left(\alpha^{0} \breve{g}_{i \mu}\right)+\partial_{i}^{0}\left(\alpha^{0} \breve{g}_{j \mu} \Gamma_{\lambda_{0}}^{j}\right)\right] \tilde{X}^{i} d^{\lambda} \wedge d^{\mu} .
$$

Hence $L_{\nu_{\tau}(X)} L_{\Gamma} \tau=0$ if and only if (5.5) is satisfied.

Thus, Lemma 5.16 implies the Proposition.

5.19. Theorem. The pair $\left(-c^{2} \tau, \Omega\right)$ is a scaled almost-cosymplectic-contact structure if and only if $g$ and $\Gamma$ fulfill the conditions (5.13).

5.4. Almost-coPoisson-Jacobi structure. First, we compute the Schouten bracket between $\gamma$ and $\Lambda$.

5.20. Lemma. For any dynamical phase connection $\gamma: \mathcal{J}_{1} \boldsymbol{E} \rightarrow \mathbb{T}^{*} \otimes T \mathcal{J}_{1} \boldsymbol{E}$, the scaled 2 -vector

$$
[\gamma, \Lambda]: \mathcal{J}_{1} \boldsymbol{E} \rightarrow \mathbb{L}^{-2} \otimes \Lambda^{2} T \mathcal{J}_{1} \boldsymbol{E}
$$

has the coordinate expression

$$
\begin{aligned}
{[\gamma, \Lambda]=[} & -\left(\alpha^{0}\right)^{2}\left(\frac{1}{2} \breve{g}^{j \lambda} \breve{\delta}_{0}^{\rho} \partial_{\rho} \hat{g}_{00}+\breve{g}^{j \lambda} \gamma_{00}^{p} \breve{g}_{0 p}+\breve{\delta}_{0}^{\lambda} \gamma_{00}^{j}\right) \\
& \left.-2 g^{0 \lambda} \gamma_{00}^{j}+\breve{\delta}_{0}^{\rho} \partial_{\rho} \breve{g}^{j \lambda}-\breve{g}^{p \lambda} \partial_{p}^{0} \gamma_{00}^{j}\right]\left(\partial_{\lambda}+\Gamma_{\lambda_{0}}^{i} \partial_{i}^{0}\right) \wedge \partial_{j}^{0} \\
+ & {\left[-\left(\alpha^{0}\right)^{2}\left(\frac{1}{2} \breve{g}^{j \rho} \breve{\delta}_{0}^{\lambda} \partial_{\rho} \hat{g}_{00}+\breve{\delta}_{0}^{\lambda} \breve{g}^{j \nu} \Gamma_{\nu 0}^{p} \breve{g}_{0 p}-\breve{\delta}_{0}^{\lambda} \breve{\delta}_{0}^{\nu} \Gamma_{\nu 0}^{j}\right)\right.} \\
& \left.+g^{\lambda \nu} \Gamma_{\nu 0}^{j}-\delta_{p}^{\lambda} \breve{g}^{j \nu} \Gamma_{\nu 0}^{p}\right] \partial_{\lambda} \wedge \partial_{j}^{0} \\
+ & {\left[-\left(\alpha^{0}\right)^{2}\left(\frac{1}{2} \breve{g}^{j \rho} \gamma_{00}^{i} \partial_{\rho} \hat{g}_{00}+\gamma_{00}^{i} \breve{g}^{j \lambda} \breve{g}_{0 p} \Gamma_{\lambda 0}^{p}\right)\right.} \\
& \left.+\breve{g}^{j \lambda} \breve{\delta}_{0}^{\rho} \partial_{\rho} \Gamma_{\lambda_{0}}^{i}+\breve{g}^{j \lambda} \gamma_{00}^{p} \partial_{p}^{0} \Gamma_{\lambda_{0}}^{i}-\breve{g}^{j \lambda} \partial_{\lambda} \gamma_{00}^{i}+\breve{g}^{i \lambda} \Gamma_{\lambda_{0}}^{p} \partial_{p}^{0} \gamma_{00}^{j}\right] \partial_{i}^{0} \wedge \partial_{j}^{0}
\end{aligned}
$$


Proof. We have

$$
\begin{aligned}
{[\gamma, \Lambda]=[} & -\left(\alpha^{0}\right)^{2}\left(\frac{1}{2}\left(\breve{g}^{j \lambda} \breve{\delta}_{0}^{\rho}+\breve{g}^{j \rho} \breve{\delta}_{0}^{\lambda}\right) \partial_{\rho} \hat{g}_{00}+\breve{g}^{j \lambda} \gamma_{00}^{p} \breve{g}_{0 p}\right. \\
& \left.+\breve{\delta}_{0}^{\lambda} \gamma_{00}^{j}+\breve{\delta}_{0}^{\lambda} \breve{g}^{j \nu} \Gamma_{\nu 0}^{p} \breve{g}_{0 p}-\breve{\delta}_{0}^{\lambda} \breve{\delta}_{0}^{\nu} \Gamma_{\nu 0}^{j}\right) \\
& \left.-2 g^{0 \lambda} \gamma_{00}^{j}+g^{\lambda \nu} \Gamma_{\nu 0}^{j}+\breve{\delta}_{0}^{\rho} \partial_{\rho} \breve{g}^{j \lambda}-\delta_{p}^{\lambda} \breve{g}^{j \nu} \Gamma_{\nu 0}^{p}-\breve{g}^{p \lambda} \partial_{p}^{0} \gamma_{00}^{j}\right] \partial_{\lambda} \wedge \partial_{j}^{0} \\
+ & -\left(\alpha^{0}\right)^{2}\left(\breve{g}^{j \lambda} \Gamma_{\lambda_{0}}^{i}\left(\frac{1}{2} \breve{\delta}_{0}^{\rho} \partial_{\rho} \hat{g}_{00}+\gamma_{00}^{p} \breve{g}_{0 p}\right)+\frac{1}{2} \breve{g}^{j \rho} \gamma_{00}^{i} \partial_{\rho} \hat{g}_{00}\right. \\
& \left.+\gamma_{00}^{i} \breve{g}^{j \lambda} \Gamma_{\lambda_{0}}^{p} \breve{g}_{0 p}+\gamma_{00}^{j} \breve{\delta}_{0}^{\lambda} \Gamma_{\lambda_{0}}^{i}\right)-2 \gamma_{00}^{j} g^{0 \lambda} \Gamma_{\lambda_{0}}^{i} \\
& +\Gamma_{\lambda 0}^{i} \breve{\delta}_{0}^{\rho} \partial_{\rho} \breve{g}^{j \lambda}+\breve{g}^{j \lambda} \breve{\delta}_{0}^{\rho} \partial_{\rho} \Gamma_{\lambda 0}^{i}+\breve{g}^{j \lambda} \gamma_{00}^{p} \partial_{p}^{0} \Gamma_{\lambda_{0}}^{i}-\breve{g}^{j \lambda} \partial_{\lambda} \gamma_{00}^{i} \\
& \left.+\breve{g}^{i \lambda} \Gamma_{\lambda 0}^{p} \partial_{p}^{0} \gamma_{00}^{j}-\breve{g}^{p \lambda} \Gamma_{\lambda_{0}}^{i} \partial_{p}^{0} \gamma_{00}^{j}\right] \partial_{i}^{0} \wedge \partial_{j}^{0} \\
= & -\left(\alpha^{0}\right)^{2}\left(\frac{1}{2} \breve{g}^{j \lambda} \breve{\delta}_{0}^{\rho} \partial_{\rho} \hat{g}_{00}+\breve{g}^{j \lambda} \gamma_{00}^{p} \breve{g}_{0 p}+\breve{\delta}_{0}^{\lambda} \gamma_{00}^{j}\right) \\
+ & \left.2 g^{0 \lambda} \gamma_{00}^{j}+\breve{\delta}_{0}^{\rho} \partial_{\rho} \breve{g}^{j \lambda}-\breve{g}^{p \lambda} \partial_{p}^{0} \gamma_{00}^{j}\right]\left(\partial_{\lambda}+\Gamma_{\lambda 0}^{i} \partial_{i}^{0}\right) \wedge \partial_{j}^{0} \\
+ & -\left(\alpha^{0}\right)^{2}\left(\frac{1}{2} \breve{g}^{j \rho} \breve{\delta}_{0}^{\lambda} \partial_{\rho} \hat{g}_{00}+\breve{\delta}_{0}^{\lambda} \breve{g}^{j \nu} \Gamma_{\nu 0}^{p} \breve{g}_{0 p}-\breve{\delta}_{0}^{\lambda} \breve{\delta}_{0}^{\nu} \Gamma_{\nu 0}^{j}\right) \\
& \left.+g^{\lambda \nu} \Gamma_{\nu 0}^{j}-\delta_{p}^{\lambda} \breve{g}^{j \nu} \Gamma_{\nu 0}^{p}\right] \partial_{\lambda} \wedge \partial_{j}^{0} \\
+ & -\left(\alpha^{0}\right)^{2}\left(\frac{1}{2} \breve{g}^{j \rho} \gamma_{00}^{i} \partial_{\rho} \hat{g}_{00}+\gamma_{00}^{i} \breve{g}^{j \lambda} \Gamma_{\lambda_{0}}^{p} \breve{g}_{0 p}\right) \\
+ & \left.\breve{g}^{j \lambda} \breve{\delta}_{0}^{\rho} \partial_{\rho} \Gamma_{\lambda 0}^{i}+\breve{g}^{j \lambda} \gamma_{00}^{p} \partial_{p}^{0} \Gamma_{\lambda_{0}}^{i}-\breve{g}^{j \lambda} \partial_{\lambda} \gamma_{00}^{i}+\breve{g}^{i \lambda} \Gamma_{\lambda_{0}}^{p} \partial_{p}^{0} \gamma_{00}^{j}\right] \partial_{i}^{0} \wedge \partial_{j}^{0}
\end{aligned}
$$

Now, let us go back to the particular case when $\gamma=: \gamma[\Gamma]$.

5.21. Proposition. We have

$$
i_{[\gamma, \Lambda]} \Omega=-i_{\gamma \wedge \Lambda} d \Omega .
$$

Proof. The Schouten bracket is characterised by the following identity [12], for each 2-form $\beta$,

$$
i_{[\gamma, \Lambda]} \beta=i_{\gamma} d i_{\Lambda} \beta-i_{\Lambda} d i_{\gamma} \beta-i_{\gamma \wedge \Lambda} d \beta
$$

Then, our claim follows from the equality $i_{\gamma} \Omega=0$ and Lemma 4.10.

5.22. Lemma. We have the coordinate expression

$$
\begin{aligned}
{[\gamma, \Lambda]=[} & -\left(\alpha^{0}\right)^{2}\left(\breve{g}^{j \lambda} \breve{\delta}_{0}^{\rho}+\breve{g}^{j \rho} \breve{\delta}_{0}^{\lambda}\right)\left(\frac{1}{2} \partial_{\rho} \hat{g}_{00}+\breve{g}_{0 p} \Gamma_{\rho 0}^{p}\right)-\delta_{p}^{\lambda} \breve{g}^{j \nu} \Gamma_{\nu 0}^{p} \\
& \left.+\breve{\delta}_{0}^{\rho}\left(\partial_{\rho} \breve{g}^{j \lambda}-g^{0 \lambda} \Gamma_{\rho 0}^{j}-\breve{g}^{p \lambda} \partial_{p}^{0} \Gamma_{\rho 0}^{j}\right)\right]\left(\partial_{\lambda}+\Gamma_{\lambda 0}^{i} \partial_{i}^{0}\right) \wedge \partial_{j}^{0} \\
& +\breve{g}^{j \lambda} \breve{\delta}_{0}^{\rho} R_{\lambda \rho 0}^{i} \partial_{i}^{0} \wedge \partial_{j}^{0} .
\end{aligned}
$$

Proof. The equalities $\gamma_{00}^{i}=\breve{\delta}_{0}^{\rho} \Gamma_{\rho 0}^{i}$ and $\partial_{p}^{0} \gamma_{00}^{i}=\delta_{p}^{\rho} \Gamma_{\rho 0}^{i}+\breve{\delta}_{0}^{\rho} \partial_{p}^{0} \Gamma_{\rho 0}^{i}$ yield

$$
\begin{aligned}
{[\gamma, \Lambda]=[} & -\left(\alpha^{0}\right)^{2}\left(\left(\breve{g}^{j \lambda} \breve{\delta}_{0}^{\rho}+\breve{g}^{j \rho} \breve{\delta}_{0}^{\lambda}\right)\left(\frac{1}{2} \partial_{\rho} \hat{g}_{00}+\breve{g}_{0 p} \Gamma_{\rho 0}^{p}\right)\right) \\
& \left.+\breve{\delta}_{0}^{\rho} \partial_{\rho} \breve{g}^{j \lambda}-g^{0 \lambda} \breve{\delta}_{0}^{\rho} \Gamma_{\rho 0}^{j}-\breve{g}^{p \lambda} \breve{\delta}_{0}^{\rho} \partial_{p}^{0} \Gamma_{\rho 0}^{j}-\delta_{p}^{\lambda} \breve{g}^{j \nu} \Gamma_{\nu 0}^{p}\right]\left(\partial_{\lambda}+\Gamma_{\lambda_{0}}^{i} \partial_{i}^{0}\right) \wedge \partial_{j}^{0} \\
+ & {\left[\breve{g}^{j \lambda} \breve{\delta}_{0}^{\rho}\left(\partial_{\rho} \Gamma_{\lambda_{0}}^{i}-\partial_{\lambda} \Gamma_{\rho 0}^{i}+\Gamma_{\rho 0}^{p} \partial_{p}^{0} \Gamma_{\lambda_{0}}^{i}-\Gamma_{\lambda 0}^{p} \partial_{p}^{0} \Gamma_{\rho 0}^{i}\right)\right] \partial_{i}^{0} \wedge \partial_{j}^{0} . }
\end{aligned}
$$


5.23. Lemma. In the adapted base (5.1) we have the coordinate expression

$$
\begin{aligned}
{[\gamma, \Lambda]=} & -\left(\alpha^{0}\right)^{2}\left(\frac{1}{2} \breve{g}^{j \rho} \partial_{\rho} \hat{g}_{00}+\breve{g}_{0 \lambda} \breve{\delta}_{0}^{\rho} \partial_{\rho} \breve{g}^{j \lambda}-\breve{\delta}_{0}^{\rho} \Gamma_{\rho 0}^{j}\right) e_{0} \wedge e_{j}^{0} \\
- & {\left[\left(\alpha^{0}\right)^{2} \hat{g}^{i j} \breve{\delta}_{0}^{\rho}\left(\frac{1}{2} \partial_{\rho} \hat{g}_{00}+\breve{g}_{0 p} \Gamma_{\rho 0}^{p}\right)\right.} \\
& \left.+\breve{g}^{j \rho} \Gamma_{\rho_{0}}^{i}-\breve{\delta}_{0}^{\rho}\left(\partial_{\rho} \hat{g}^{j i}-\breve{g}^{i 0} \Gamma_{\rho 0}^{j}-\hat{g}^{p i} \partial_{p}^{0} \Gamma_{\rho 0}^{j}\right)\right] e_{i} \wedge e_{j}^{0} \\
& -\breve{g}^{j \lambda} \breve{\delta}_{0}^{\rho} R_{\lambda \rho 0}^{i} e_{i}^{0} \wedge e_{j}^{0} .
\end{aligned}
$$

Proof. It follows from (5.19) and (5.1).

5.24. Lemma. We have the coordinate expression

$$
\begin{aligned}
\gamma \wedge \Lambda^{\sharp}\left(L_{\gamma} \tau\right)= & \left(\alpha^{0}\right)^{2}\left(\breve{\delta}_{0}^{\lambda}\left(\frac{1}{2} \breve{g}^{j \rho} \partial_{\rho} \hat{g}_{00}+\breve{g}_{0 \rho} \breve{\delta}_{0}^{\sigma} \partial_{\sigma} \breve{g}^{j \rho}-\breve{\delta}_{0}^{\sigma} \Gamma_{\sigma 0}^{j}\right) \partial_{\lambda} \wedge \partial_{j}^{0}\right. \\
& \left.+\breve{\delta}_{0}^{\sigma} \Gamma_{\sigma 0}^{i} \breve{g}^{j \rho}\left(\frac{1}{2} \partial_{\rho} \hat{g}_{00}-\breve{\delta}_{0}^{\tau} \partial_{\tau} \breve{g}_{0 \rho}\right) \partial_{i}^{0} \wedge \partial_{j}^{0}\right),
\end{aligned}
$$

which, in the adapted base (5.1), reads as

$$
\gamma \wedge \Lambda^{\sharp}\left(L_{\gamma} \tau\right)=\left(\alpha^{0}\right)^{2}\left(\frac{1}{2} \breve{g}^{j \rho} \partial_{\rho} \hat{g}_{00}+\breve{g}_{0 \rho} \breve{\delta}_{0}^{\sigma} \partial_{\sigma} \breve{g}^{j \rho}-\breve{\delta}_{0}^{\sigma} \Gamma_{\sigma 0}^{j}\right) e_{0} \wedge e_{j}^{0} .
$$

Proof. We have

$$
\begin{aligned}
L_{\gamma} \tau & =-\alpha^{0} \breve{\delta}_{0}^{\rho}\left[\partial_{\rho}\left(\alpha^{0} \breve{g}_{0 \lambda}\right)-\partial_{\lambda}\left(\alpha^{0} \breve{g}_{0 \rho}\right)+\partial_{p}^{0}\left(\alpha^{0} \breve{g}_{0 \lambda}\right) \Gamma_{\rho 0}^{p}\right] d^{\lambda} \\
& =-\left(\alpha^{0}\right)^{2} \breve{\delta}_{0}^{\rho}\left[\partial_{\rho} \breve{g}_{0 \lambda}-\partial_{\lambda} \breve{g}_{0 \rho}+\breve{g}_{p \lambda} \Gamma_{\rho 0}^{p}+\frac{1}{2}\left(\alpha^{0}\right)^{2}\left(\breve{g}_{0 \lambda} \partial_{\rho} \hat{g}_{00}-\breve{g}_{0 \rho} \partial_{\lambda} \hat{g}_{00}\right)\right] d^{\lambda} .
\end{aligned}
$$

Then, we have

$$
\begin{aligned}
\Lambda^{\sharp}\left(L_{\gamma} \tau\right) & =-\frac{\alpha^{0}}{c} \breve{g}^{i \lambda} \breve{\delta}_{0}^{\rho}\left[\partial_{\rho} \breve{g}_{0 \lambda}-\partial_{\lambda} \breve{g}_{0 \rho}+\breve{g}_{p \lambda} \Gamma_{\rho 0}^{p}+\frac{1}{2}\left(\alpha^{0}\right)^{2}\left(\breve{g}_{0 \lambda} \partial_{\rho} \hat{g}_{00}-\breve{g}_{0 \rho} \partial_{\lambda} \hat{g}_{00}\right)\right] \partial_{i}^{0} \\
& =\frac{\alpha^{0}}{c}\left[\frac{1}{2} \breve{g}^{i \lambda} \partial_{\lambda} \hat{g}_{00}+\breve{g}_{0 \lambda} \breve{\delta}_{0}^{\rho} \partial_{\rho} \breve{g}^{i \lambda}-\Gamma_{\rho 0}^{i} \breve{\delta}_{0}^{\rho}\right] \partial_{i}^{0}
\end{aligned}
$$

and

$$
\gamma \wedge \Lambda^{\sharp}\left(L_{\gamma} \tau\right)=\left(\alpha^{0}\right)^{2} \breve{\delta}_{0}^{\lambda}\left(\partial_{\lambda}+\Gamma_{\lambda_{0}}^{i} \partial_{i}^{0}\right) \wedge\left(\frac{1}{2} \breve{g}^{j \rho} \partial_{\rho} \hat{g}_{00}+\breve{g}_{0 \rho} \breve{\delta}_{0}^{\sigma} \partial_{\sigma} \breve{g}^{j \rho}-\breve{\delta}_{0}^{\sigma} \Gamma_{\sigma 0}^{j}\right) \partial_{j}^{0} .
$$

5.25. Corollary. In the adapted base (5.21) we have the coordinate expression

$$
\begin{aligned}
{\left[-\frac{1}{c^{2}} \gamma, \Lambda\right]-} & \frac{1}{c^{2}} \gamma \wedge \Lambda^{\sharp}\left(L_{\gamma} \tau\right)=\left[\frac{\left(\alpha^{0}\right)^{2}}{c^{2}} \hat{g}^{i j} \breve{\delta}_{0}^{\rho}\left(\frac{1}{2} \partial_{\rho} \hat{g}_{00}+\breve{g}_{0 p} \Gamma_{\rho 0}^{p}\right)\right. \\
& \left.+\frac{1}{c^{2}}\left(\breve{g}^{j \rho} \Gamma_{\rho 0}^{i}-\breve{\delta}_{0}^{\rho}\left(\partial_{\rho} \hat{g}^{j i}-\breve{g}^{i 0} \Gamma_{\rho 0}^{j}-\hat{g}^{p i} \partial_{p}^{0} \Gamma_{\rho 0}^{j}\right)\right)\right] e_{i} \wedge e_{j}^{0} \\
& -\frac{1}{c^{2}} \breve{g}^{j \lambda} \breve{\delta}_{0}^{\rho} R_{\lambda \rho 0}^{i} e_{i}^{0} \wedge e_{j}^{0} .
\end{aligned}
$$

Proof. It follows from (5.20) and (5.22).

Then, we compute the Schouten bracket between $\Lambda$ and $\Lambda$.

5.26. Lemma. The scaled 3-vector

$$
[\Lambda, \Lambda]: \mathcal{\partial}_{1} \boldsymbol{E} \rightarrow\left(\mathbb{T}^{2} \otimes \mathbb{L}^{-4}\right) \otimes \Lambda^{3} T \mathcal{J}_{1} \boldsymbol{E},
$$


has the coordinate expression

$$
\begin{aligned}
{[\Lambda, \Lambda]=} & \frac{2}{c^{2}}\left[\breve{\delta}_{0}^{\lambda} \breve{g}^{j \mu} \partial_{\lambda} \wedge \partial_{\mu} \wedge \partial_{i}^{0}+\left(\breve{\delta}_{0}^{\lambda} \breve{g}^{j \rho}-\breve{\delta}_{0}^{\rho} \breve{g}^{j \lambda}\right) \Gamma_{\rho 0}^{i} \partial_{\lambda} \wedge \partial_{i}^{0} \wedge \partial_{j}^{0}\right. \\
& \left.+\breve{\delta}_{0}^{\sigma} \breve{g}^{k \rho} \Gamma_{\sigma 0}^{i} \Gamma_{\rho 0}^{j} \partial_{i}^{0} \wedge \partial_{j}^{0} \wedge \partial_{k}^{0}\right] \\
+ & \frac{2}{c^{2}}\left[\breve{g}^{j \rho} \breve{g}^{k \lambda}\left(\frac{1}{2} \partial_{\rho} \hat{g}_{00}+\breve{g}_{0 p} \Gamma_{\rho 0}^{p}\right)\right. \\
& \left.+\frac{1}{\left(\alpha^{0}\right)^{2}} \breve{g}^{k \rho}\left(\partial_{\rho} \breve{g}^{j \lambda} g^{0 \lambda} \Gamma_{\rho 0}^{j}-\breve{g}^{p \lambda} \partial_{p}^{0} \Gamma_{\rho 0}^{j}\right)\right]\left(\partial_{\lambda}+\Gamma_{\lambda_{0}}^{i} \partial_{i}^{0}\right) \wedge \partial_{j}^{0} \wedge \partial_{k}^{0} \\
+ & \frac{1}{\left(c \alpha^{0}\right)^{2}} \breve{g}^{i \rho} \breve{g}^{j \sigma} R_{\rho \sigma 0}^{k} \partial_{i}^{0} \wedge \partial_{j}^{0} \wedge \partial_{k}^{0} .
\end{aligned}
$$

Proof. We have

$$
\begin{aligned}
& {[\Lambda, \Lambda]=\frac{2}{c^{2}} \breve{\delta}_{0}^{\lambda} \breve{g}^{j \mu} \partial_{\lambda} \wedge \partial_{\mu} \wedge \partial_{i}^{0}} \\
& +\frac{2}{c^{2}}\left[\breve{g}^{i \rho} \breve{g}^{j \lambda}\left(\frac{1}{2} \partial_{\rho} \hat{g}_{00}+\breve{g}_{0 p} \Gamma_{\rho 0}^{p}\right)+\breve{\delta}_{0}^{\lambda} \breve{g}^{j \rho} \Gamma_{\rho 0}^{i}-\breve{\delta}_{0}^{\rho} \breve{g}^{j \lambda} \Gamma_{\rho 0}^{i}\right. \\
& \left.+\frac{1}{\left(\alpha^{0}\right)^{2}} \breve{g}^{j \rho}\left(\partial_{\rho} \breve{g}^{i \lambda}-g^{0 \lambda} \Gamma_{\rho 0}^{i}-\breve{g}^{p \lambda} \partial_{p}^{0} \Gamma_{\rho 0}^{i}\right)\right] \partial_{\lambda} \wedge \partial_{i}^{0} \wedge \partial_{j}^{0} \\
& +\frac{2}{c^{2}}\left[\breve{g}^{i \sigma} \breve{g}^{j \rho} \Gamma_{\rho 0}^{k}\left(\frac{1}{2} \partial_{\sigma} \hat{g}_{00}+\breve{g}_{0 p} \Gamma_{\sigma 0}^{p}\right)+\breve{\delta}_{0}^{\sigma} \breve{g}^{k \rho} \Gamma_{\sigma 0}^{i} \Gamma_{\rho_{0}}^{j}\right. \\
& +\frac{1}{\left(\alpha^{0}\right)^{2}}\left(\breve{g}^{i \sigma} \partial_{\sigma}\left(\breve{g}^{k \rho} \Gamma_{\rho 0}^{j}\right)+g^{0 \rho} \breve{g}^{i \sigma} \Gamma_{\rho 0}^{j} \Gamma_{\sigma 0}^{k}\right. \\
& \left.\left.+\left(\breve{g}^{i \sigma} \Gamma_{\sigma 0}^{p}-\breve{g}^{p \sigma} \Gamma_{\sigma 0}^{i}\right) \partial_{p}^{0}\left(\breve{g}^{k \rho} \Gamma_{\rho 0}^{j}\right)\right)\right] \partial_{i}^{0} \wedge \partial_{j}^{0} \wedge \partial_{k}^{0} \\
& =\frac{2}{c^{2}} \breve{\delta}_{0}^{\lambda} \breve{g}^{j \mu} \partial_{\lambda} \wedge \partial_{\mu} \wedge \partial_{i}^{0} \\
& +\frac{2}{c^{2}}\left[\breve{g}^{i \rho} \breve{g}^{j \lambda}\left(\frac{1}{2} \partial_{\rho} \hat{g}_{00}+\breve{g}_{0 p} \Gamma_{\rho 0}^{p}\right)+\left(\breve{\delta}_{0}^{\lambda} \breve{g}^{j \rho}-\breve{\delta}_{0}^{\rho} \breve{g}^{j \lambda}\right) \Gamma_{\rho 0}^{i}\right. \\
& \left.+\frac{1}{\left(\alpha^{0}\right)^{2}} \breve{g}^{j \rho}\left(\partial_{\rho} \breve{g}^{i \lambda}-g^{0 \lambda} \Gamma_{\rho 0}^{i}-\breve{g}^{p \lambda} \partial_{p}^{0} \Gamma_{\rho 0}^{i}\right)\right] \partial_{\lambda} \wedge \partial_{i}^{0} \wedge \partial_{j}^{0} \\
& +\frac{2}{c^{2}}\left[\breve{g}^{i \sigma} \breve{g}^{j \rho} \Gamma_{\rho 0}^{k}\left(\frac{1}{2} \partial_{\sigma} \hat{g}_{00}+\breve{g}_{0 p} \Gamma_{\sigma 0}^{p}\right)+\breve{\delta}_{0}^{\sigma} \breve{g}^{k \rho} \Gamma_{\sigma 0}^{i} \Gamma_{\rho 0}^{j}\right. \\
& +\frac{1}{\left(\alpha^{0}\right)^{2}}\left(-\frac{1}{2} \breve{g}^{i \sigma} \breve{g}^{k \rho} R_{\sigma \rho 0}^{j}\right. \\
& \left.\left.+\breve{g}^{i \sigma} \Gamma_{\rho 0}^{j}\left(\partial_{\sigma} \breve{g}^{k \rho}-g^{0 \rho} \Gamma_{\sigma 0}^{k}-\breve{g}^{p \rho} \partial_{p}^{0} \Gamma_{\sigma 0}^{k}\right)\right)\right] \partial_{i}^{0} \wedge \partial_{j}^{0} \wedge \partial_{k}^{0} .
\end{aligned}
$$

5.27. Lemma. In the adapted base (5.1) we have the coordinate expression

$$
\begin{aligned}
{[\Lambda, \Lambda]=} & \frac{2}{c^{2}}\left[\hat{g}^{k j} e_{0} \wedge e_{j} \wedge e_{k}^{0}+\breve{g}^{k \rho}\left(\Gamma_{\rho 0}^{j}-\breve{g}_{0 \lambda} \partial_{\rho} \breve{g}^{j \lambda}\right) e_{0} \wedge e_{j}^{0} \wedge e_{k}^{0}\right. \\
+ & \left(\hat{g}^{k i} \breve{g}^{j \rho}\left(\frac{1}{2} \partial_{\rho} \hat{g}_{00}+\breve{g}_{0 p} \Gamma_{\rho 0}^{p}\right)\right. \\
& \left.+\frac{1}{\left(\alpha^{0}\right)^{2}} \breve{g}^{k \rho}\left(\partial_{\rho} \hat{g}^{j i}-\breve{g}^{i 0} \Gamma_{\rho 0}^{j}-\hat{g}^{p i} \partial_{p}^{0} \Gamma_{\rho 0}^{j}\right)\right) e_{i} \wedge e_{j}^{0} \wedge e_{k}^{0} \\
& \left.+\frac{1}{\left(\alpha^{0}\right)^{2}} \breve{g}^{i \rho} \breve{g}^{j \sigma} R_{\rho \sigma 0}^{k} e_{i}^{0} \wedge e_{j}^{0} \wedge e_{k}^{0}\right] .
\end{aligned}
$$

Proof. It follows from (5.24) and (5.1).

5.28. Lemma. In the adapted base (5.1) we have the coordinate expression

$$
\gamma \wedge\left(\Lambda^{\sharp} \otimes \Lambda^{\sharp}\right)(d \tau)=\frac{1}{c^{2}}\left[\hat{g}^{j k} e_{0} \wedge e_{j} \wedge e_{k}^{0}\right.
$$




$$
\left.+\breve{g}^{j \rho}\left(\breve{g}_{0 \sigma} \partial_{\rho} \breve{g}^{k \sigma}-\breve{g}^{j \rho} \Gamma_{\rho 0}^{k}\right) e_{0} \wedge e_{j}^{0} \wedge e_{k}^{0}\right]
$$

Proof. We have

$$
\begin{aligned}
\left(\Lambda^{\sharp} \otimes \Lambda^{\sharp}\right)(d \tau)= & \frac{1}{c^{3} \alpha^{0}}\left[\breve{g}^{j \lambda} \partial_{\lambda} \wedge \partial_{j}^{0}\right. \\
& \left.-\left(\breve{g}^{i \lambda} \breve{g}^{j \mu} \partial_{\lambda} \breve{g}_{0 \mu}+\breve{g}^{i \rho} \Gamma_{\rho_{0}}^{j}-\breve{g}^{j \rho} \Gamma_{\rho 0}^{i}\right) \partial_{i}^{0} \wedge \partial_{j}^{0}\right]
\end{aligned}
$$

Then

$$
\begin{aligned}
\gamma \wedge\left(\Lambda^{\sharp} \otimes \Lambda^{\sharp}\right)(d \tau)= & \frac{1}{c^{2}}\left(\breve { \delta } _ { 0 } ^ { \nu } ( \partial _ { \nu } + \Gamma _ { \nu 0 } ^ { k } \partial _ { k } ^ { 0 } ) \wedge \left[\breve{g}^{j \lambda} \partial_{\lambda} \wedge \partial_{j}^{0}\right.\right. \\
& \left.-\left(\breve{g}^{i \lambda} \breve{g}^{j \mu} \partial_{\lambda} \breve{g}_{0 \mu}+\breve{g}^{i \rho} \Gamma_{\rho 0}^{j}-\breve{g}^{j \rho} \Gamma_{\rho 0}^{i}\right) \partial_{i}^{0} \wedge \partial_{j}^{0}\right] \\
= & \frac{1}{c^{2}}\left[\breve{\delta}_{0}^{\lambda} \breve{g}^{k \mu} \partial_{\lambda} \wedge \partial_{\mu} \wedge \partial_{k}^{0}\right. \\
& +\left(-\breve{\delta}_{0}^{\lambda}\left(\breve{g}^{j \rho} \breve{g}^{k \sigma} \partial_{\rho} \breve{g}_{0 \sigma}+\breve{g}^{j \rho} \Gamma_{\rho 0}^{k}-\breve{g}^{k \rho} \Gamma_{\rho 0}^{j}\right)+\breve{\delta}_{0}^{\rho} \breve{g}^{j \lambda} \Gamma_{\rho 0}^{k}\right) \partial_{\lambda} \wedge \partial_{j}^{0} \wedge \partial_{k}^{0} \\
& \left.-\breve{\delta}_{0}^{\rho} \Gamma_{\rho 0}^{k}\left(\breve{g}^{i \lambda} \breve{g}^{j \mu} \partial_{\lambda} \breve{g}_{0 \mu}+\breve{g}^{i \rho} \Gamma_{\rho_{0}}^{j}-\breve{g}^{j \rho} \Gamma_{\rho 0}^{i}\right) \partial_{i}^{0} \wedge \partial_{j}^{0} \wedge \partial_{k}^{0}\right] .
\end{aligned}
$$

Then, in the adapted base (5.1) we obtain the Lemma.

5.29. Lemma. In the adapted base (5.1) we have the coordinate expression

$$
\begin{aligned}
& {[\Lambda, \Lambda]-2 \gamma \wedge\left(\Lambda^{\sharp} \otimes \Lambda^{\sharp}\right)(d \tau)=\frac{2}{c^{2}}\left[\left(\hat{g}^{k i} \breve{g}^{j \rho}\left(\frac{1}{2} \partial_{\rho} \hat{g}_{00}+\breve{g}_{0 p} \Gamma_{\rho 0}^{p}\right)\right.\right.} \\
& \left.+\frac{1}{\left(\alpha^{0}\right)^{2}} \breve{g}^{k \rho}\left(\partial_{\rho} \hat{g}^{j i}-\breve{g}^{i 0} \Gamma_{\rho 0}^{j}-\hat{g}^{p i} \partial_{p}^{0} \Gamma_{\rho 0}^{j}\right)\right) e_{i} \wedge e_{j}^{0} \wedge e_{k}^{0} \\
& \left.+\frac{1}{\left(\alpha^{0}\right)^{2}} \breve{g}^{i \rho} \breve{g}^{j \sigma} R_{\rho \sigma 0}^{k} e_{i}^{0} \wedge e_{j}^{0} \wedge e_{k}^{0}\right]
\end{aligned}
$$

Proof. It follows from (5.25) and (5.26).

5.30. Lemma. The pair $\left(-\frac{1}{c^{2}} \gamma, \Lambda\right)$ is a scaled almost-coPoisson-Jacobi structure along with the fundamental 1 -form $-c^{2} \tau$ if and only if the following identities are satisfied

$$
\begin{array}{r}
\left(\alpha^{0}\right)^{2} \hat{g}^{j i} \breve{\delta}_{0}^{\rho}\left(\frac{1}{2} \partial_{\rho} \hat{g}_{00}+\breve{g}_{0 p} \Gamma_{\rho 0}^{p}\right) \\
-\breve{\delta}_{0}^{\rho}\left(\partial_{\rho} \hat{g}^{j i}-\breve{g}^{i 0} \Gamma_{\rho 0}^{j}-\hat{g}^{p i} \partial_{p}^{0} \Gamma_{\rho 0}^{j}\right)+\breve{g}^{j \rho} \Gamma_{\rho_{0}}^{i}=0, \\
\breve{g}^{j \lambda} \breve{\delta}_{0}^{\rho} R_{\lambda \rho 0}^{i}-\breve{g}^{i \lambda} \breve{\delta}_{0}^{\rho} R_{\lambda \rho 0}^{j}=0, \\
\left(\alpha^{0}\right)^{2}\left(\breve{g}^{j \rho} \hat{g}^{k i}-\breve{g}^{k \rho} \hat{g}^{j i}\right)\left(\frac{1}{2} \partial_{\rho} \hat{g}_{00}+\breve{g}_{0 p} \Gamma_{\rho 0}^{p}\right) \\
+\breve{g}^{k \rho}\left(\partial_{\rho} \hat{g}^{j i}-\breve{g}^{i 0} \Gamma_{\rho 0}^{j}-\hat{g}^{p i} \partial_{p}^{0} \Gamma_{\rho 0}^{j}\right)-\breve{g}^{j \rho}\left(\partial_{\rho} \hat{g}^{k i}-\breve{g}^{i 0} \Gamma_{\rho 0}^{k}-\hat{g}^{p i} \partial_{p}^{0} \Gamma_{\rho 0}^{k}\right)=0, \\
\breve{g}^{i \sigma} \breve{g}^{k \rho} R_{\rho \sigma 0}^{j}+\breve{g}^{j \sigma} \breve{g}^{i \rho} R_{\rho \sigma 0}^{k}+\breve{g}^{k \sigma} \breve{g}^{j \rho} R_{\rho \sigma 0}^{i}
\end{array}
$$

Proof. $\left(-\frac{1}{c^{2}} \gamma, \Lambda\right)$ is a scaled almost-coPoisson-Jacobi structure along with the fundamental 1-form $-c^{2} \tau$ if and only if $\left[-\frac{1}{c^{2}} \gamma, \Lambda\right]=-\left(-\frac{1}{c^{2}} \gamma\right) \wedge \Lambda^{\sharp}\left(L_{-\frac{1}{c^{2}} \gamma}\left(-c^{2} \tau\right)\right)=\frac{1}{c^{2}} \gamma \wedge \Lambda^{\sharp}\left(L_{\Gamma} \tau\right)$ and $[\Lambda, \Lambda]=-\frac{2}{c^{2}} \gamma \wedge\left(\Lambda^{\sharp} \otimes \Lambda^{\sharp}\right)\left(-c^{2} d \tau\right)=2 \gamma \wedge\left(\Lambda^{\sharp} \otimes \Lambda^{\sharp}\right)(d \tau)$. 
But, in virtue of Corollary 5.25, $\left[-\frac{1}{c^{2}} \gamma, \Lambda\right]=\frac{1}{c^{2}} \gamma \wedge \Lambda^{\sharp}\left(L_{\gamma} \tau\right)$ if and only if (5.28) and (5.29) are satisfied. Moreover, in virtue of Lemma 5.29, $[\Lambda, \Lambda]=2 \gamma \wedge\left(\Lambda^{\sharp} \otimes \Lambda^{\sharp}\right)(d \tau)$ if and only if (5.30) and (5.31) are satisfied.

5.31. Theorem. The pair $\left(-\frac{1}{c^{2}} \gamma, \Lambda\right)$ is a scaled almost-coPoisson-Jacobi structure along with the fundamental 1-form $-c^{2} \tau$ if and only if the conditions (5.13) are satisfied.

Proof. 1st proof. By Theorem 1.1 the structure $\left(-c^{2} \tau, \Omega\right)$ is a scaled almost-cosymplecticcontact structure if and only if the dual structure $\left(-\frac{1}{c^{2}} \gamma, \Lambda\right)$ is a scaled almost-coPoissonJacobi structure along with the fundamental 1-form $-c^{2} \tau$. On the other hand, according Theorem 5.19, $\left(-c^{2} \tau, \Omega\right)$ is a scaled almost-cosymplectic-contact structure if and only if the conditions (5.13) are satisfied.

2nd proof. Next, we prove Thorem directly in local coordinates.

From Lemma 5.30 it folows that $\left(-\frac{1}{c^{2}} \gamma, \Lambda\right)$ is a scaled almost-coPoisson-Jacobi structure along with the fundamental 1 -form $-c^{2} \tau$ if and only if (5.28) - (5.31) are satisfied.

We have the coordinate expression (5.15) of $L_{R} \tau$ and, in the adapted base (5.2), we obtain

$$
\begin{aligned}
L_{R} \tau= & -\frac{2 \alpha^{0}}{c} \hat{g}_{i p} \breve{\delta}_{0}^{\rho} R_{\rho k 0}^{p} \epsilon^{i} \wedge \epsilon^{0} \wedge \epsilon^{k} \\
& -\frac{\alpha^{0}}{c} \hat{g}_{i p}\left(R_{j k 0}^{p}+\left(\alpha^{0}\right)^{2} \breve{\delta}_{0}^{\rho}\left(\breve{g}_{0 j} R_{\rho k 0}^{p}+\breve{g}_{0 k} R_{j \rho 0}^{p}\right)\right) \epsilon^{i} \wedge \epsilon^{j} \wedge \epsilon^{k}
\end{aligned}
$$

Then $\sharp\left(L_{R} \tau\right)$, in the adapted base (5.1), has the coordinate expression

$$
\begin{aligned}
& \sharp\left(L_{R} \tau\right)=-\frac{2 \hbar \alpha^{0}}{m c^{5}} \hat{g}^{p k} \breve{\delta}_{0}^{\rho} R_{\rho p}^{i} e_{i}^{0} \wedge e_{0} \wedge e_{k}^{0} \\
& -\frac{1}{c^{4}\left(\alpha^{0}\right)^{2}}\left(\hat{g}^{p j} \hat{g}^{q k} R_{p q 0}^{i}+\breve{g}^{0 j} \hat{g}^{p k} \breve{\delta}_{0}^{\rho} R_{\rho p 0}^{i}+\breve{g}^{0 k} \hat{g}^{p j} \breve{\delta}_{0}^{\rho} R_{p \rho 0}^{i}\right) e_{i}^{0} \wedge e_{j}^{0} \wedge e_{k}^{0} \text {. }
\end{aligned}
$$

The identity $\hat{g}^{i p}=\breve{\delta}_{\lambda}^{p} \breve{g}^{i \lambda}$ implies

$$
\hat{g}^{i p} \breve{\delta}_{0}^{\rho} R_{\rho p 0}^{j}=\breve{g}^{i \lambda} \breve{\delta}_{0}^{\rho} R_{\rho \lambda 0}^{j}-\breve{g}^{0 i} \breve{\delta}_{0}^{\rho} \breve{\delta}_{0}^{\sigma} R_{\rho \sigma 0}^{i}=\breve{g}^{i \lambda} \breve{\delta}_{0}^{\rho} R_{\rho \lambda 0}^{j}
$$

and, similarly,

$$
\hat{g}^{p j} \hat{g}^{q k} R_{p q 0}^{i}=\breve{g}^{j \lambda} \breve{g}^{k \mu}\left(R_{\lambda \mu 0}^{i}-\delta_{\lambda}^{0} \breve{\delta}_{0}^{\rho} R_{\rho \mu 0}^{i}-\delta_{\mu}^{0} \breve{\delta}_{0}^{\rho} R_{\lambda \rho 0}^{i}\right)
$$

Then,

$$
\begin{aligned}
\sharp\left(L_{R} \tau\right)= & -\frac{2 \hbar \alpha^{0}}{m c^{5}} \breve{g}^{k \lambda} \breve{\delta}_{0}^{\rho} R_{\rho \lambda 0}{ }^{i} e_{i}^{0} \wedge e_{0} \wedge e_{k}^{0} \\
& -\frac{1}{c^{4}\left(\alpha^{0}\right)^{2}}\left(\breve{g}^{j \lambda} \breve{g}^{k \mu}\left(R_{\lambda \mu 0}^{i}-\delta_{\lambda}^{0} \breve{\delta}_{0}^{\rho} R_{\rho \mu 0}^{i}-\delta_{\mu}^{0} \breve{\delta}_{0}^{\rho} R_{\lambda \rho 0}{ }^{i}\right)\right. \\
& \left.\quad+\breve{g}^{0 j} \breve{g}^{k \mu} \breve{\delta}_{0}^{\rho} R_{\rho \mu 0}^{i}+\breve{g}^{0 k} \breve{g}^{j \lambda} \breve{\delta}_{0}^{\rho} R_{\lambda \rho 0}{ }^{i}\right) e_{i}^{0} \wedge e_{j}^{0} \wedge e_{k}^{0} \\
= & -\frac{2 \hbar \alpha^{0}}{m c^{5}} \breve{g}^{k \lambda} \breve{\delta}_{0}^{\rho} R_{\rho \lambda 0}{ }^{i} e_{i}^{0} \wedge e_{0} \wedge e_{k}^{0}-\frac{1}{c^{4}\left(\alpha^{0}\right)^{2}} \breve{g}^{j \lambda} \breve{g}^{k \mu} R_{\lambda \mu 0}^{i} e_{i}^{0} \wedge e_{j}^{0} \wedge e_{k}^{0} .
\end{aligned}
$$

Then, $L_{R} \tau=0$ if and only if $\sharp\left(L_{R} \tau\right)=0$, i.e., if and only if (5.29) and (5.31) are satisfied.

Further, let us consider a scaled vector field $X \in \sec \left(\boldsymbol{E}, \mathbb{L}^{-2} \otimes T \boldsymbol{E}\right)$. Then, $\nu_{\tau}(X) \in$ $\sec \left(\mathcal{J}_{1} \boldsymbol{E}, \mathbb{T} \otimes \mathbb{L}^{-2} \otimes V \mathcal{J}_{1} \boldsymbol{E}\right) \subset \sec \left(\mathcal{J}_{1} \boldsymbol{E}, V_{\tau} \mathcal{J}_{1} \boldsymbol{E}\right)$ and there exists a unique 1 -form $\omega \in$ $\sec \left(\mathcal{J}_{1} \boldsymbol{E}, V_{\gamma}^{*} \mathcal{J}_{1} \boldsymbol{E}\right)$ such that $\nu_{\tau}(X)=\Lambda^{\sharp}(\omega)$. In the adapted base (5.1)

$$
\nu_{\tau}(X)=\frac{1}{c \alpha^{0}} \widetilde{X}^{i} e_{i}^{0}=\frac{1}{c \alpha^{0}} \hat{g}^{i p} \widetilde{\omega}_{p} e_{i}^{0}=\Lambda^{\sharp}(\omega) .
$$


Next, let us refer to the coordinate expression 5.17 of $L_{\nu_{\tau}(X)} L_{\Gamma} \tau$. Then, in the adapted base (5.2), we obtain

$$
\begin{aligned}
L_{\Lambda^{\sharp}(\omega)} L_{\Gamma} \tau=- & \frac{1}{c^{2} \alpha^{0}} \breve{\delta}_{0}^{\rho}\left[\partial_{\rho}\left(\alpha^{0} \breve{g}_{p j}\right)+\partial_{p}^{0}\left(\alpha^{0} \breve{g}_{j q} \Gamma_{\rho 0}^{q}\right)\right. \\
& \left.-\partial_{j}\left(\alpha^{0} \breve{g}_{p \rho}\right)-\partial_{p}^{0}\left(\alpha^{0} \breve{g}_{q \mu} \Gamma_{j 0}^{q}\right)\right] \hat{g}^{i p} \widetilde{\omega}_{i} \epsilon^{0} \wedge \epsilon^{j} \\
- & \frac{1}{c^{2} \alpha^{0}}\left[\partial_{j}\left(\alpha^{0} \breve{g}_{p k}\right)+\partial_{p}^{0}\left(\alpha^{0} \breve{g}_{q k} \Gamma_{j 0}^{q}\right)+\left(\alpha^{0}\right)^{2} \breve{g}_{0 j} \breve{\delta}_{0}^{\rho}\left(\partial_{\rho}\left(\alpha^{0} \breve{g}_{p k}\right)\right.\right. \\
& \left.\left.\left.+\partial_{p}^{0}\left(\alpha^{0} \breve{g}_{k q} \Gamma_{\rho 0}^{q}\right)\right)+\left(\alpha^{0}\right)^{2} \breve{g}_{0 k} \breve{\delta}_{0}^{\rho} \partial_{p}^{0}\left(\alpha^{0} \breve{g}_{q \mu} \Gamma_{j 0}^{q}\right)\right)\right] \hat{g}^{p i} \widetilde{\omega}_{i} \epsilon^{j} \wedge \epsilon^{k} .
\end{aligned}
$$

Then ${ }^{\sharp}\left(L_{\Lambda^{\sharp}(\omega)} L_{\Gamma} \tau\right)$ has the coordinate expression, in the adapted base (5.1) ,

$$
\begin{aligned}
\sharp\left(L_{\Lambda}^{\sharp(\omega)} L_{\Gamma} \tau\right)=- & \frac{\hbar}{m c^{5}} \hat{g}^{k s} \hat{g}^{p i} \breve{\delta}_{0}^{\rho}\left[\partial_{\rho}\left(\alpha^{0} \breve{g}_{p s}\right)+\partial_{p}^{0}\left(\alpha^{0} \breve{g}_{s q} \Gamma_{\rho 0}^{q}\right)\right. \\
& \left.-\partial_{s}\left(\alpha^{0} \breve{g}_{p \rho}\right)-\partial_{p}^{0}\left(\alpha^{0} \breve{g}_{q \rho} \Gamma_{s 0}^{q}\right)\right] \widetilde{\omega}_{i} e_{0} \wedge e_{j}^{0} \\
- & \frac{1}{c^{4}\left(\alpha^{0}\right)^{3}} \hat{g}^{p i} \hat{g}^{j r} \hat{g}^{k s}\left[\partial_{p}^{0}\left(\alpha^{0} \breve{g}_{q s} \Gamma_{r 0}^{q}\right)+\left(\alpha^{0}\right)^{2} \breve{g}_{0 s} \breve{\delta}_{0}^{\rho} \partial_{p}^{0}\left(\alpha^{0} \breve{g}_{q \mu} \Gamma_{r 0}^{q}\right)\right) \\
& \left.+\partial_{r}\left(\alpha^{0} \breve{g}_{p s}\right)+\left(\alpha^{0}\right)^{2} \breve{g}_{0 r} \breve{\delta}_{0}^{\rho}\left(\partial_{\rho}\left(\alpha^{0} \breve{g}_{p s}\right)+\partial_{p}^{0}\left(\alpha^{0} \breve{g}_{s q} \Gamma_{\rho 0}^{q}\right)\right)\right] \widetilde{\omega}_{i} e_{j}^{0} \wedge e_{k}^{0} \\
=- & \frac{\hbar \alpha^{0}}{m c^{5}}\left[\left(\alpha^{0}\right)^{2} \hat{g}^{j i} \breve{\delta}_{0}^{\rho}\left(\frac{1}{2} \partial_{\rho} \hat{g}_{00}+\breve{g}_{0 p} \Gamma_{\rho 0}^{p}\right)\right. \\
& \left.-\breve{\delta}_{0}^{\rho}\left(\partial_{\rho} \hat{g}^{j i}-\breve{g}^{i 0} \Gamma_{\rho 0}^{j}-\hat{g}^{p i} \partial_{p}^{0} \Gamma_{\rho 0}^{j}\right)+\breve{g}^{j \rho} \Gamma_{\rho 0}^{i}\right] \widetilde{\omega}_{i} e_{0} \wedge e_{j}^{0} \\
- & \frac{1}{c^{4}\left(\alpha^{0}\right)^{2}}\left[\left(\alpha^{0}\right)^{2} \hat{g}^{k i} \breve{g}^{j \rho}\left(\frac{1}{2} \partial_{\rho} \hat{g}_{00}+\breve{g}_{0 q} \Gamma_{\rho 0}^{q}\right)\right. \\
& \left.-\breve{g}^{j \rho}\left(\partial_{\rho} \hat{g}^{k i}-\breve{g}^{i 0} \Gamma_{\rho 0}^{k}-\hat{g}^{p i} \partial_{p}^{0} \Gamma_{\rho 0}^{k}\right)\right] \widetilde{\omega}_{i} e_{j}^{0} \wedge e_{k}^{0}
\end{aligned}
$$

Then $L_{\nu_{\tau}(X)} L_{\Gamma} \tau=0$ if and only if $L_{\Lambda^{\sharp}(\omega)} L_{\Gamma} \tau=0$, i.e., if and only if the equalities (5.28) and (5.30) are satisfied.

We can summarize the main results of the previous two sections as follows.

5.32. Theorem. The following assertions are equivalent.

(1) $L_{\nu_{\tau}(X)} L_{\Gamma} \tau=0, \forall X \in \sec (\boldsymbol{E}, T \boldsymbol{E})$, and $L_{R} \tau=0$.

(2) $d \Omega=0$, i.e. $\left(-c^{2} \tau, \Omega\right)$ is a (scaled) almost-cosymplectic-contact structure.

(3) $\left.\left[-\frac{1}{c^{2}} \gamma, \Lambda\right]=\frac{1}{c^{2}} \gamma \wedge \Lambda^{\sharp}\left(L_{\gamma} \tau\right)\right)$ and $\left.[\Lambda, \Lambda]=2 \gamma \wedge\left(\Lambda^{\sharp} \otimes \Lambda^{\sharp}\right)(d \tau)\right)$, i.e. $\left(-\frac{1}{c^{2}} \gamma, \Lambda\right)$ is a (scaled) almost-coPoisson-Jacobi structure along with the fundamental 1-form $-c^{2} \tau$.

Moreover, the almost-cosymplectic-contact pair $\left(-c^{2} \tau, \Omega\right)$ and the almost-coPoissonJacobi 3-plet $\left(-\frac{1}{c^{2}} \gamma, \Lambda,-c^{2} \tau\right)$ are mutually dual.

5.5. Contact structure. Next, we analyse the conditions by which the pair $\left(-c^{2} \tau, \Omega\right)$ is a contact pair, i.e. $\Omega=-c^{2} d \tau$. As a direct consequence of Theorem 5.19 we obtain that if $L_{\Gamma} \tau=0$, then the pair $\left(-c^{2} \tau, \Omega\right)$ is a scaled almost-cosymplectic-contact structure. Indeed, the condition $L_{\Gamma} \tau=0$ implies $L_{\nu_{\tau}(X)} L_{\Gamma} \tau=0$ and $L_{R} \tau=0$, because of $L_{R} \tau=$ $-L_{[\Gamma, \Gamma]} \tau=-2 L_{\Gamma} L_{\Gamma} \tau$. Hence, by Theorem 5.19, if $L_{\Gamma} \tau=0$, then the pair $\left(-c^{2} \tau, \Omega\right)$ is a scaled almost-cosymplectic-contact structure. But, in this case we can prove more.

5.33. Lemma. We have

$$
d \Omega=c^{2} d L_{\Gamma} \tau
$$


Proof. We have

$$
\begin{aligned}
d L_{\Gamma} \tau= & -\frac{1}{c}\left[\partial_{\nu}\left(\partial_{\lambda}\left(\alpha^{0} \breve{g}_{0 \mu}\right)+\Gamma_{\lambda_{0}}^{j} \partial_{j}^{0}\left(\alpha^{0} \breve{g}_{0 \mu}\right)\right) d^{\nu} \wedge d^{\lambda} \wedge d^{\mu}\right. \\
& \left.+\partial_{i}^{0}\left(\partial_{\lambda}\left(\alpha^{0} \breve{g}_{0 \mu}\right)+\Gamma_{\lambda_{0}}^{j} \partial_{j}^{0}\left(\alpha^{0} \breve{g}_{0 \mu}\right)\right) d_{0}^{i} \wedge d^{\lambda} \wedge d^{\mu}\right] \\
= & -\frac{1}{c}\left[\partial_{\nu}\left(\alpha^{0} \breve{g}_{j \mu} \Gamma_{\lambda 0}^{j}\right) d^{\nu} \wedge d^{\lambda} \wedge d^{\mu}\right. \\
& \left.+\left(\partial_{\lambda}\left(\alpha^{0} \breve{g}_{i \mu}\right)+\partial_{i}^{0}\left(\alpha^{0} \breve{g}_{j \mu} \Gamma_{\lambda_{0}}^{j}\right)\right) d_{0}^{i} \wedge d^{\lambda} \wedge d^{\mu}\right] .
\end{aligned}
$$

Hence, we obtain the Lemma by comparing the above equality with (5.7).

5.34. Corollary. The difference $\Omega-c^{2} L_{\Gamma} \tau$ is an exact form. More precisely, we have

$$
\Omega-c^{2} L_{\Gamma} \tau=-c^{2} d \tau
$$

Proof. We have

$$
\begin{aligned}
\Omega-c^{2} L_{\Gamma} \tau & =c \alpha^{0} \breve{g}_{i \mu}\left(d_{0}^{i}-\Gamma_{\lambda_{0}}^{i} d^{\lambda}\right) \wedge d^{\mu}+c\left(\partial_{\lambda}\left(\alpha^{0} \breve{g}_{0 \mu}\right)+\alpha^{0} \breve{g}_{p \mu} \Gamma_{\lambda_{0}}^{p}\right) d^{\lambda} \wedge d^{\mu} \\
& =c\left(\alpha^{0} \breve{g}_{i \mu} d_{0}^{i} \wedge d^{\mu}+\partial_{\lambda}\left(\alpha^{0} \breve{g}_{0 \mu}\right) d^{\lambda} \wedge d^{\mu}\right) \\
& =c\left(\partial_{i}^{0}\left(\alpha^{0} \breve{g}_{0 \mu}\right) d_{0}^{i} \wedge d^{\mu}+\partial_{\lambda}\left(\alpha^{0} \breve{g}_{0 \mu}\right) d^{\lambda} \wedge d^{\mu}\right)=-c^{2} d \tau .
\end{aligned}
$$

5.35. Theorem. The pair $\left(-c^{2} \tau, \Omega\right)$ is a scaled contact structure if and only if $L_{\Gamma} \tau=$ 0 .

5.6. Jacobi structure. Next, we analyse conditions for the pair $\left(-\frac{1}{c^{2}} \gamma, \Lambda\right)$ to be a scaled Jacobi structure.

5.36. Lemma. We have the coordinate expression

$$
\begin{aligned}
\gamma \wedge \Lambda= & \breve{\delta}_{0}^{\lambda} \breve{g}^{i \mu} \partial_{\lambda} \wedge \partial_{\mu} \wedge \partial_{i}^{0}+\left(\breve{\delta}_{0}^{\lambda} \breve{g}^{j \rho}-\breve{\delta}_{0}^{\rho} \breve{g}^{j \lambda}\right) \Gamma_{\rho 0}^{i} \partial_{\lambda} \wedge \partial_{i}^{0} \wedge \partial_{j}^{0} \\
& +\breve{\delta}_{0}^{\rho} \breve{g}^{k \sigma} \Gamma_{\rho 0}^{i} \Gamma_{\sigma 0}^{j} \partial_{i}^{0} \wedge \partial_{j}^{0} \wedge \partial_{k}^{0}
\end{aligned}
$$

and, in the adapted base (5.1),

$$
\gamma \wedge \Lambda=\hat{g}^{i j} e_{0} \wedge e_{i} \wedge e_{i}^{0}
$$

Proof. It follows from the coordinate expressions

$$
\gamma=c \alpha^{0} \breve{\delta}_{0}^{\lambda}\left(\partial_{\lambda}+\Gamma_{\lambda_{0}}^{i} \partial_{i}^{0}\right)=c \alpha^{0} e_{0}
$$

and

$$
\Lambda=\frac{1}{c \alpha^{0}} \breve{g}^{j \lambda}\left(\partial_{\lambda}+\Gamma_{\lambda_{0}}^{i} \partial_{i}^{0}\right) \wedge \partial_{j}^{0}=\frac{1}{c \alpha^{0}} \hat{g}^{i j} e_{i} \wedge e_{j}^{0}
$$

5.37. Lemma. In the adapted base (5.1) we have the coordinate expression

$$
\begin{aligned}
{[\Lambda, \Lambda]-} & \frac{2}{c^{2}} \gamma \wedge \Lambda=\frac{2}{c^{2}}\left[\breve{g}^{k \rho}\left(\Gamma_{\rho 0}^{j}-\breve{g}_{0 \lambda} \partial_{\rho} \breve{g}^{j \lambda}\right) e_{0} \wedge e_{j}^{0} \wedge e_{k}^{0}\right. \\
+ & \left(\hat{g}^{k i} \breve{g}^{j \rho}\left(\frac{1}{2} \partial_{\rho} \hat{g}_{00}+\breve{g}_{0 p} \Gamma_{\rho 0}^{p}\right)\right. \\
& \left.+\frac{1}{\left(\alpha^{0}\right)^{2}} \breve{g}^{k \rho}\left(\partial_{\rho} \hat{g}^{j i}-\breve{g}^{i 0} \Gamma_{\rho 0}^{j}-\hat{g}^{p i} \partial_{p}^{0} \Gamma_{\rho 0}^{j}\right)\right) e_{i} \wedge e_{j}^{0} \wedge e_{k}^{0}
\end{aligned}
$$




$$
\left.+\frac{1}{\left(\alpha^{0}\right)^{2}} \breve{g}^{i \rho} \breve{g}^{j \sigma} R_{\rho \sigma 0}^{k} e_{i}^{0} \wedge e_{j}^{0} \wedge e_{k}^{0}\right]
$$

Proof. It follows from (5.25) and (5.33).

5.38. Lemma. $L_{\Gamma} \tau=0$ if and only if the identities

$$
\begin{aligned}
\frac{1}{2} \breve{g}^{j \rho} \partial_{\rho} \hat{g}_{00}+\breve{\delta}_{0}^{\rho}\left(\breve{g}_{0 \lambda} \partial_{\rho} \breve{g}^{j \lambda}-\Gamma_{\rho 0}^{j}\right) & =0 \\
\breve{g}^{i \rho}\left(\breve{g}_{0 \lambda} \partial_{\rho} \breve{g}^{j \lambda}-\Gamma_{\rho 0}^{j}\right)-\breve{g}^{j \rho}\left(\breve{g}_{0 \lambda} \partial_{\rho} \breve{g}^{i \lambda}-\Gamma_{\rho 0}^{i}\right) & =0 .
\end{aligned}
$$

are satisfied.

Proof. In the adapted base (5.1) we have

$$
\begin{aligned}
\sharp\left(L_{\Gamma} \tau\right)= & \frac{\hbar\left(\alpha^{0}\right)^{2}}{m c^{4}}\left(\frac{1}{2} \breve{g}^{j \rho} \partial_{\rho} \hat{g}_{00}+\breve{\delta}_{0}^{\rho}\left(\breve{g}_{0 \lambda} \partial_{\rho} \breve{g}^{j \lambda}-\Gamma_{\rho 0}^{j}\right)\right) e_{0} \wedge e_{j}^{0} \\
& +\frac{1}{c^{3} \alpha^{0}} \breve{g}^{i \rho}\left(\breve{g}_{0 \lambda} \partial_{\rho} \breve{g}^{j \lambda}-\Gamma_{\rho 0}^{j}\right) e_{i}^{0} \wedge e_{j}^{0} .
\end{aligned}
$$

Then, $L_{\Gamma} \tau=0$ if and only if $\sharp\left(L_{\Gamma} \tau\right)=0$, i.e. if and only if the identities (5.35) and (5.36) are satisfied.

5.39. Lemma. The pair $\left(-\frac{1}{c^{2}} \gamma, \Lambda\right)$ is a scaled Jacobi structure if and only if the identities (5.35) and (5.36) are satisfied.

Proof. The pair $\left(-\frac{1}{c^{2}} \gamma, \Lambda\right)$ is a scaled Jacobi structure if and only if $\left[-\frac{1}{c^{2}} \gamma, \Lambda\right]=0$ and $[\Lambda, \Lambda]=\frac{2}{c^{2}} \gamma \wedge \Lambda$. According to the coordinate expressions in Lemmas 5.23 and 5.37, these conditions are satisfied if and only if the identities (5.28) - (5.31), (5.35) and (5.36) are satisfied. But, we can prove that the identities (5.35) and (5.36) imply the identities (5.28) - (5.31).

First, let us prove that the identities (5.35) and (5.36) imply the identities (5.29) and (5.31). But it follows from the fact that the identities (5.35) and (5.36) are satisfied if and only if $L_{\Gamma} \tau=0$. But, in this case $L_{R} \tau=-L_{[\Gamma, \Gamma]} \tau=-2 L_{\Gamma} L_{\Gamma} \tau=0$, which is equivalent to the identities (5.29) and (5.31).

Next, let us recall the identities $\hat{g}^{i p} \delta_{p}^{\rho}=\breve{g}^{i \rho}-\breve{g}^{i 0} \breve{\delta}_{0}^{\rho}, \hat{g}^{i p} g_{p \lambda}=\breve{\delta}_{\lambda}^{i}-\breve{g}^{i 0} \breve{g}_{0 \lambda}$ and $g^{0 \lambda}=$ $(\alpha)^{2}\left(\breve{g}_{0 p} \breve{g}^{p \lambda}-\breve{\delta}_{0}^{\lambda}\right)$. Hence, if we apply the operator $\hat{g}^{i p} \partial_{p}^{0}$ on the left hand side of the identity (5.35) and we suppose that the identities (5.35) and (5.36) are satisfied, then we obtain

$$
\begin{aligned}
0= & -\frac{1}{2} \hat{g}^{i j} g^{0 \rho} \partial_{\rho} \hat{g}_{00}+\hat{g}^{i p} \breve{g}^{j \rho} \partial_{\rho} \breve{g}_{0 p}+\left(\hat{g}^{i p} \delta_{p}^{\rho} \breve{g}_{0 \lambda}+\hat{g}^{i p} \breve{\delta}_{0}^{\rho} g_{p \lambda}\right) \partial_{\rho} \breve{g}^{j \lambda} \\
& -\hat{g}^{i p} \delta_{p}^{\rho} \Gamma_{\rho 0}^{j}-\hat{g}^{i j} \breve{g}_{0 \lambda} \breve{\delta}_{0}^{\rho} \partial_{\rho} g^{0 \lambda}-\hat{g}^{i p} \breve{\delta}_{0}^{\rho} \partial_{p}^{0} \Gamma_{\rho 0}^{j} \\
= & -\frac{1}{2} \hat{g}^{i j} g^{0 \rho} \partial_{\rho} \hat{g}_{00}-\breve{g}^{j \rho} \breve{g}_{0 p} \breve{\delta}_{\lambda}^{p} \partial_{\rho} \breve{g}^{i \lambda}-\breve{g}^{j \rho} \breve{g}^{i 0} \partial_{\rho} \hat{g}_{00}+\frac{1}{\left(\alpha^{0}\right)^{2}} \breve{g}^{j \rho} \partial_{\rho} \breve{g}^{i 0}-\hat{g}^{i j} \breve{g}_{0 \lambda} \breve{\delta}_{0}^{\rho} \partial_{\rho} g^{0 \lambda} \\
& -\hat{g}^{i p} \breve{\delta}_{0}^{\rho} \partial_{p}^{0} \Gamma_{\rho 0}^{j}+\left(\breve{g}^{i \rho} \breve{g}_{0 \lambda}-2 \breve{g}^{i 0} \breve{\delta}_{0}^{\rho} \breve{g}_{0 \lambda}+\breve{\delta}_{\lambda}^{i} \breve{\delta}_{0}^{\rho}\right) \partial_{\rho} \breve{g}^{j \lambda}-\left(\breve{g}^{i \rho}-\breve{g}^{i 0} \breve{\delta}_{0}^{\rho}\right) \Gamma_{\rho 0}^{j} \\
= & -\frac{1}{2} \hat{g}^{i j} g^{0 \rho} \partial_{\rho} \hat{g}_{00}-\breve{g}^{j \rho} \breve{g}^{i 0} \partial_{\rho} \hat{g}_{00}-\breve{g}_{0 \lambda}\left(\breve{g}^{j \rho} \partial_{\rho} \breve{g}^{i \lambda}-\breve{g}^{i \rho} \partial_{\rho} \breve{g}^{j \lambda}\right)-\hat{g}^{i j} \breve{g}_{0 \lambda} \breve{\delta}_{0}^{\rho} \partial_{\rho} g^{0 \lambda} \\
& -2 \breve{g}^{i 0} \breve{\delta}_{0}^{\rho} \breve{g}_{0 \lambda} \partial_{\rho} \breve{g}^{j \lambda}+\breve{\delta}_{0}^{\rho}\left(\partial_{\rho} \hat{g}^{i j}+\breve{g}^{i 0} \Gamma_{\rho 0}^{j}-\hat{g}^{i p} \partial_{p}^{0} \Gamma_{\rho 0}^{j}\right)-\breve{g}^{i \rho} \Gamma_{\rho 0}^{j} \\
= & -\left(\alpha^{0}\right)^{2} \hat{g}^{i j} \breve{\delta}_{0}^{\rho}\left(\frac{1}{2} \partial_{\rho} \hat{g}_{00}+\breve{g}_{0 p} \Gamma_{\rho 0}^{p}\right)+\breve{\delta}_{0}^{\rho}\left(\partial_{\rho} \hat{g}^{i j}-\breve{g}^{i 0} \Gamma_{\rho 0}^{j}-\hat{g}^{i p} \partial_{p}^{0} \Gamma_{\rho 0}^{j}\right)-\breve{g}^{j \rho} \Gamma_{\rho 0}^{i} .
\end{aligned}
$$


Hence, the identities (5.35) and (5.36) imply the identity (5.28).

Finally, we apply the same method on the left hand side of the identity (5.36) and obtain

$$
\begin{aligned}
& 0=-\hat{g}^{i k} g^{0 \rho}\left(\breve{g}_{0 \lambda} \partial_{\rho} \breve{g}^{j \lambda}-\Gamma_{\rho_{0}}^{j}\right)+\breve{g}^{k \rho}\left(\hat{g}^{p i} g_{p \lambda} \partial_{\rho} \breve{g}^{j \lambda}-\hat{g}^{i j} \breve{g}_{0 \lambda} \partial_{\rho} g^{0 \lambda}-\hat{g}^{i p} \partial_{p}^{0} \Gamma_{\rho_{0}}^{j}\right) \\
& +\hat{g}^{i j} g^{0 \rho}\left(\breve{g}_{0 \lambda} \partial_{\rho} \breve{g}^{k \lambda}-\Gamma_{\rho 0}^{k}\right)-\breve{g}^{j \rho}\left(\hat{g}^{p i} g_{p \lambda} \partial_{\rho} \breve{g}^{k \lambda}-\hat{g}^{i k} \breve{g}_{0 \lambda} \partial_{\rho} g^{0 \lambda}-\hat{g}^{i p} \partial_{p}^{0} \Gamma_{\rho 0}^{k}\right) \\
& =\hat{g}^{i k} g^{0 \rho}\left(\breve{g}^{j \lambda} \partial_{\rho} \breve{g}_{0 \lambda}+\Gamma_{\rho_{0}}^{j}\right)+\breve{g}^{k \rho}\left(\partial_{\rho} \hat{g}^{i j}+\hat{g}^{i j} g^{0 \lambda} \partial_{\rho} \breve{g}_{0 \lambda}-\hat{g}^{i p} \partial_{p}^{0} \Gamma_{\rho_{0}}^{j}\right) \\
& -\hat{g}^{i j} g^{0 \rho}\left(\breve{g}^{k \lambda} \partial_{\rho} \breve{g}_{0 \lambda}+\Gamma_{\rho 0}^{k}\right)-\breve{g}^{j \rho}\left(\partial_{\rho} \hat{g}^{i k}+\hat{g}^{i k} g^{0 \lambda} \partial_{\rho} \breve{g}_{0 \lambda}-\hat{g}^{i p} \partial_{p}^{0} \Gamma_{\rho 0}^{k}\right) \\
& -\breve{g}^{i 0}\left(\breve{g}^{k \rho} \breve{g}_{0 \lambda} \partial_{\rho} \breve{g}^{j \lambda}-\breve{g}^{j \rho} \breve{g}_{0 \lambda} \partial_{\rho} \breve{g}^{k \lambda}\right) \\
& =\left(\hat{g}^{i k} g^{0 \rho} \breve{g}^{j \lambda}+\hat{g}^{i j} g^{0 \lambda} \breve{g}^{k \rho}\right) \partial_{\rho} \breve{g}_{0 \lambda}+\hat{g}^{i k} g^{0 \rho} \Gamma_{\rho 0}^{j}+\breve{g}^{k \rho}\left(\partial_{\rho} \hat{g}^{i j}-\breve{g}^{i 0} \Gamma_{\rho_{0}}^{j}-\hat{g}^{i p} \partial_{p}^{0} \Gamma_{\rho_{0}}^{j}\right) \\
& -\left(\hat{g}^{i j} g^{0 \rho} \breve{g}^{k \lambda}+\hat{g}^{i k} g^{0 \lambda} \breve{g}^{j \rho}\right) \partial_{\rho} \breve{g}_{0 \lambda}-\hat{g}^{i j} g^{0 \rho} \Gamma_{\rho 0}^{k}-\breve{g}^{j \rho}\left(\partial_{\rho} \hat{g}^{i k}-\breve{g}^{i 0} \Gamma_{\rho 0}^{k}-\hat{g}^{i p} \partial_{p}^{0} \Gamma_{\rho 0}^{k}\right) \\
& =g^{0 \rho}\left(\hat{g}^{i k} \breve{g}^{j \lambda}-\hat{g}^{i j} \breve{g}^{k \lambda}\right) \partial_{\rho} \breve{g}_{0 \lambda}-g^{0 \lambda}\left(\hat{g}^{i k} \breve{g}^{j \rho}-\hat{g}^{i j} \breve{g}^{k \rho}\right) \partial_{\rho} \breve{g}_{0 \lambda}+g^{0 \rho}\left(\hat{g}^{i k} \Gamma_{\rho 0}^{j}-\hat{g}^{i j} \Gamma_{\rho 0}^{k}\right) \\
& +\breve{g}^{k \rho}\left(\partial_{\rho} \hat{g}^{i j}-\breve{g}^{i 0} \Gamma_{\rho 0}^{j}-\hat{g}^{i p} \partial_{p}^{0} \Gamma_{\rho 0}^{j}\right)-\breve{g}^{j \rho}\left(\partial_{\rho} \hat{g}^{i k}-\breve{g}^{i 0} \Gamma_{\rho 0}^{k}-\hat{g}^{i p} \partial_{p}^{0} \Gamma_{\rho 0}^{k}\right) \\
& =\left(\alpha^{0}\right)^{2}\left(\breve{\delta}_{0}^{\lambda}-\breve{g}_{0 p} \breve{g}^{p \lambda}\right)\left(\hat{g}^{i k} \breve{g}^{j \rho}-\hat{g}^{i j} \breve{g}^{k \rho}\right) \partial_{\rho} \breve{g}_{0 \lambda} \\
& -\left(\alpha^{0}\right)^{2}\left(\breve{\delta}_{0}^{\rho}-\breve{g}_{0 p} \breve{g}^{p \rho}\right)\left(\breve{g}_{0 \lambda}\left(\hat{g}^{i j} \partial_{\rho} \breve{g}^{k \lambda}-\hat{g}^{i k} \partial_{\rho} \breve{g}^{j \lambda}\right)+\hat{g}^{i k} \Gamma_{\rho_{0}^{j}}^{j}-\hat{g}^{i j} \Gamma_{\rho 0}^{k}\right) \\
& +\breve{g}^{k \rho}\left(\partial_{\rho} \hat{g}^{i j}-\breve{g}^{i 0} \Gamma_{\rho 0}^{j}-\hat{g}^{i p} \partial_{p}^{0} \Gamma_{\rho 0}^{j}\right)-\breve{g}^{j \rho}\left(\partial_{\rho} \hat{g}^{i k}-\breve{g}^{i 0} \Gamma_{\rho 0}^{k}-\hat{g}^{i p} \partial_{p}^{0} \Gamma_{\rho 0}^{k}\right) \\
& =\left(\alpha^{0}\right)^{2}\left(\hat{g}^{i k} \breve{g}^{j \rho}-\hat{g}^{i j} \breve{g}^{k \rho}\right) \partial_{\rho} \hat{g}_{00}-\left(\alpha^{0}\right)^{2} \breve{g}_{0 p} \breve{g}_{0 \lambda}\left(\hat{g}^{i k} \breve{g}^{j \rho}-\hat{g}^{i j} \breve{g}^{k \rho}\right) \partial_{\rho} \breve{g}^{p \lambda} \\
& -\left(\alpha^{0}\right)^{2}\left(\breve{\delta}_{0}^{\rho}-\breve{g}_{0 p} \breve{g}^{p \rho}\right)\left(\breve{g}_{0 \lambda}\left(\hat{g}^{i j} \partial_{\rho} \breve{g}^{k \lambda}-\hat{g}^{i k} \partial_{\rho} \breve{g}^{j \lambda}\right)+\hat{g}^{i k} \Gamma_{\rho 0}^{j}-\hat{g}^{i j} \Gamma_{\rho 0}^{k}\right) \\
& +\breve{g}^{k \rho}\left(\partial_{\rho} \hat{g}^{i j}-\breve{g}^{i 0} \Gamma_{\rho 0}^{j}-\hat{g}^{i p} \partial_{p}^{0} \Gamma_{\rho 0}^{j}\right)-\breve{g}^{j \rho}\left(\partial_{\rho} \hat{g}^{i k}-\breve{g}^{i 0} \Gamma_{\rho 0}^{k}-\hat{g}^{i p} \partial_{p}^{0} \Gamma_{\rho 0}^{k}\right) \\
& =\left(\alpha^{0}\right)^{2}\left(\hat{g}^{i k} \breve{g}^{j \rho}-\hat{g}^{i j} \breve{g}^{k \rho}\right) \partial_{\rho} \hat{g}_{00} \\
& -\left(\alpha^{0}\right)^{2} \breve{\delta}_{0}^{\rho}\left(\hat{g}^{i j}\left(\breve{g}_{0 \lambda} \partial_{\rho} \breve{g}^{k \lambda}-\Gamma_{\rho 0}^{k}\right)-\hat{g}^{i k}\left(\breve{g}_{0 \lambda} \partial_{\rho} \breve{g}^{j \lambda}-\Gamma_{\rho_{0}}^{j}\right)\right) \\
& +\left(\alpha^{0}\right)^{2} \breve{g}_{0 p}\left(\hat{g}^{i k}\left(\breve{g}^{j \rho} \breve{g}_{0 \lambda} \partial_{\rho} \breve{g}^{p \lambda}-\breve{g}^{p \rho} \breve{g}_{0 \lambda} \partial_{\rho} \breve{g}^{j \lambda}\right)-\hat{g}^{i j}\left(\breve{g}^{k \rho} \breve{g}_{0 \lambda} \partial_{\rho} \breve{g}^{p \lambda}-\breve{g}^{p \rho} \breve{g}_{0 \lambda} \partial_{\rho} \breve{g}^{k \lambda}\right)\right) \\
& +\breve{g}^{k \rho}\left(\partial_{\rho} \hat{g}^{i j}-\breve{g}^{i 0} \Gamma_{\rho 0}^{j}-\hat{g}^{i p} \partial_{p}^{0} \Gamma_{\rho 0}^{j}\right)-\breve{g}^{j \rho}\left(\partial_{\rho} \hat{g}^{i k}-\breve{g}^{i 0} \Gamma_{\rho 0}^{k}-\hat{g}^{i p} \partial_{p}^{0} \Gamma_{\rho 0}^{k}\right) \\
& =+\left(\alpha^{0}\right)^{2}\left(\hat{g}^{i k} \breve{g}^{j \rho}-\hat{g}^{i j} \breve{g}^{k \rho}\right) \partial_{\rho} \hat{g}_{00}+\frac{1}{2}\left(\alpha^{0}\right)^{2}\left(\hat{g}^{i j} \breve{g}^{k \rho}-\hat{g}^{i k} \breve{g}^{j \rho}\right) \partial_{\rho} \hat{g}_{00} \\
& +\left(\alpha^{0}\right)^{2} \breve{g}_{0 p}\left(\hat{g}^{i k}\left(\breve{g}^{j \rho} \Gamma_{\rho 0}^{p}-\breve{g}^{p \rho} \Gamma_{\rho 0}^{j}\right)-\hat{g}^{i j}\left(\breve{g}^{k \rho} \Gamma_{\rho 0}^{p}-\breve{g}^{p \rho} \Gamma_{\rho 0}^{k}\right)\right) \\
& +\breve{g}^{k \rho}\left(\partial_{\rho} \hat{g}^{i j}-\breve{g}^{i 0} \Gamma_{\rho 0}^{j}-\hat{g}^{i p} \partial_{p}^{0} \Gamma_{\rho 0}^{j}\right)-\breve{g}^{j \rho}\left(\partial_{\rho} \hat{g}^{i k}-\breve{g}^{i 0} \Gamma_{\rho 0}^{k}-\hat{g}^{i p} \partial_{p}^{0} \Gamma_{\rho 0}^{k}\right) \\
& =\left(\alpha^{0}\right)^{2}\left(\hat{g}^{i k} \breve{g}^{j \rho}-\hat{g}^{i j} \breve{g}^{k \rho}\right)\left(\frac{1}{2} \partial_{\rho} \hat{g}_{00}+\breve{g}_{0 p} \Gamma_{\rho 0}^{p}\right) \\
& +\breve{g}^{k \rho}\left(\partial_{\rho} \hat{g}^{i j}-\breve{g}^{i 0} \Gamma_{\rho 0}^{j}-\hat{g}^{i p} \partial_{p}^{0} \Gamma_{\rho 0}^{j}\right)-\breve{g}^{j \rho}\left(\partial_{\rho} \hat{g}^{i k}-\breve{g}^{i 0} \Gamma_{\rho 0}^{k}-\hat{g}^{i p} \partial_{p}^{0} \Gamma_{\rho 0}^{k}\right) \text {. }
\end{aligned}
$$

So, the identities (5.35) and (5.36) imply the identity (5.30). 
5.40. Theorem. The pair $\left(-\frac{1}{c^{2}} \gamma, \Lambda\right)$ is a scaled Jacobi structure if and only if

$$
L_{\Gamma} \tau=0 \text {. }
$$

Proof. 1st proof. By Theorem 1.1 the structure $\left(-c^{2} \tau, \Omega\right)$ is a scaled contact structure if and only if the dual structure $\left(-\frac{1}{c^{2}} \gamma, \Lambda\right)$ is a scaled Jacobi structure. But, according to Theorem 5.35, $\left(-c^{2} \tau, \Omega\right)$ is a scaled contact structure if and only if $L_{\Gamma} \tau=0$.

2nd proof. By Lemma $5.39\left(-\frac{1}{c^{2}} \gamma, \Lambda\right)$ is a scaled Jacobi structute if and only if the identities (5.35) and (5.36) are satisfied but, by Lemma 5.38, it is equivalent with $L_{\Gamma} \tau=$ 0 .

We can summarize the above results as follows.

5.41. Theorem. The following assertions are equivalent.

(1) $L_{\Gamma} \tau=0$.

(2) $\Omega=-c^{2} d \tau$, i.e. $\left(-c^{2} \tau, \Omega\right)$ is a (scaled) contact structure.

(3) $\left[-\frac{1}{c^{2}} \gamma, \Lambda\right]=0$ and $[\Lambda, \Lambda]=\frac{2}{c^{2}} \gamma \wedge \Lambda$, i.e. $\left(-\frac{1}{c^{2}} \gamma, \Lambda\right)$ is a (scaled regular) Jacobi structure.

Moreover, the contact structure $\left(-c^{2} \tau, \Omega\right)$ and the Jacobi structure $\left(-\frac{1}{c^{2}} \gamma, \Lambda\right)$ are mutually dual.

\section{Contact and Jacobi structures: linear CASE}

Next, we specialise the results of the previous section concerning the contact and Jacobi structures, by considering the phase connection $\Gamma=\chi(K)$ induced by a linear spacetime connection $K$. Thus, in this section, we refer to the induced objects $\Omega=\Omega[g, K]=\Omega[g, \chi(K)], \Lambda=\Lambda[g, K]=\Lambda[g, \chi(K)], \gamma=\gamma[K]=\gamma[\chi(K)]$.

6.1. Covariant derivatives and the adapted base. We start with some technical formulas concerning the covariant derivatives $\nabla$ with respect to the connection $K$ of the metric in the orthogonal adapted bases $\left(b_{0}, b_{1}\right)$ and $\left(\beta^{0}, \beta^{i}\right)$.

6.1. Lemma. We have

$$
\begin{aligned}
& \nabla_{\lambda} \breve{g}_{0 \mu}=:\left(\nabla_{\lambda} g\right)\left(b_{0}, \partial_{\mu}\right)=\breve{\delta}_{0}^{\nu} \nabla_{\lambda} g_{\nu \mu}=\partial_{\lambda} \breve{g}_{0 \mu}+g_{\rho \mu} K_{\lambda}{ }^{\rho}{ }_{\sigma} \breve{\delta}_{0}^{\sigma}+\breve{g}_{0 \rho} K_{\lambda}{ }^{\rho}{ }_{\mu}, \\
& \nabla_{\lambda} \breve{g}_{i \mu}=:\left(\nabla_{\lambda} g\right)\left(b_{i}, \partial_{\mu}\right)=\nabla_{\lambda} g_{i \mu}+\left(\alpha^{0}\right)^{2} \breve{g}_{0 i} \nabla_{\lambda} \breve{g}_{0 \mu} \\
& \nabla_{\lambda} \breve{g}^{0 \mu}=:\left(\nabla_{\lambda} \bar{g}\right)\left(\beta^{0}, d^{\mu}\right)=-\left(\alpha^{0}\right)^{2} \breve{g}_{0 \nu}\left(\partial_{\lambda} g^{\nu \mu}-g^{\rho \mu} K_{\lambda}{ }^{\nu}{ }_{\rho}-g^{\nu \rho} K_{\lambda}{ }^{\mu}{ }_{\rho}\right), \\
& \nabla_{\lambda} \breve{g}^{i \mu}=:\left(\nabla_{\lambda} \bar{g}\right)\left(\beta^{i}, d^{\mu}\right)=\breve{\delta}_{\nu}^{i} \nabla_{\lambda} g^{\nu \mu}=\partial_{\lambda} \breve{g}^{i \mu}-\breve{\delta}_{\nu}^{i} g^{\rho \mu} K_{\lambda}{ }^{\nu}{ }_{\rho}-\breve{g}^{i \rho} K_{\lambda}{ }^{\mu}{ }_{\rho} .
\end{aligned}
$$

Moreover, we have the identities

$$
\begin{aligned}
\breve{g}_{0 \mu} \nabla_{\lambda} \breve{g}^{0 \mu} & =-\breve{g}^{0 \mu} \nabla_{\lambda} \breve{g}_{0 \mu}, & \breve{g}_{0 \mu} \nabla_{\lambda} \breve{g}^{i \mu} & =-\breve{g}^{i \mu} \nabla_{\lambda} \breve{g}_{0 \mu}, \\
\breve{g}_{i \mu} \nabla_{\lambda} \breve{g}^{0 \mu} & =-\breve{g}^{0 \mu} \nabla_{\lambda} \breve{g}_{i \mu}, & \breve{g}_{i \mu} \nabla_{\lambda} \breve{g}^{j \mu} & =-\breve{g}^{j \mu} \nabla_{\lambda} \breve{g}_{i \mu} .
\end{aligned}
$$

Proof. We have

$$
\nabla_{\lambda} g=\nabla_{\lambda} g_{\nu \mu} d^{\nu} \otimes d^{\mu}=\nabla_{\lambda} g_{\nu \mu}\left(\breve{\delta}_{0}^{\nu} \beta^{0}+\left(\delta_{i}^{\nu}+\left(\alpha^{0}\right)^{2} \breve{g}_{0 i} \breve{\delta}_{0}^{\nu}\right) \beta^{i}\right) \otimes d^{\mu} .
$$


Then, we obtain

$$
\left(\nabla_{\lambda} g\right)\left(b_{0}, \partial_{\mu}\right)=\breve{\delta}_{0}^{\nu} \nabla_{\lambda} g_{\nu \mu} \quad \text { and } \quad\left(\nabla_{\lambda} g\right)\left(b_{i}, \partial_{\mu}\right)=\left(\delta_{i}^{\nu}+\left(\alpha^{0}\right)^{2} \breve{g}_{0 i} \breve{\delta}_{0}^{\nu}\right) \nabla_{\lambda} g_{\nu \mu} .
$$

Analogously, we have

$$
\nabla_{\lambda} \bar{g}=\nabla_{\lambda} g^{\nu \mu} \partial_{\nu} \otimes \partial_{\mu}=\nabla_{\lambda} g^{\nu \mu}\left(\breve{\delta}_{\nu}^{i} b_{i}-\left(\alpha^{0}\right)^{2} \breve{g}_{0 \nu} b_{0}\right) \otimes \partial_{\mu} .
$$

Then, we obtain

$$
\nabla_{\lambda} g\left(\beta^{0}, d^{\mu}\right)=-\left(\alpha^{0}\right)^{2} \breve{g}_{0 \nu} \nabla_{\lambda} g^{\nu \mu} \quad \text { and } \quad \nabla_{\lambda} g\left(\beta^{i}, d^{\mu}\right)=\breve{\delta}_{\nu}^{i} \nabla_{\lambda} g^{\nu \mu} .
$$

\subsection{Lemma. We have}

$$
\begin{aligned}
& \nabla_{\lambda} \hat{g}_{00}=:\left(\nabla_{\lambda} g\right)\left(b_{0}, b_{0}\right)=-\nabla_{\lambda}\left(\alpha^{0}\right)^{-2}=\partial_{\lambda} \hat{g}_{00}+2 \breve{g}_{0 \rho} K_{\lambda}{ }^{\rho}{ }_{\sigma} \breve{\delta}_{0}^{\sigma}, \\
& \nabla_{\lambda} \hat{g}_{i 0}=:\left(\nabla_{\lambda} g\right)\left(b_{i}, b_{0}\right)=\nabla_{\lambda} \breve{g}_{0 i}+\left(\alpha^{0}\right)^{2} \breve{g}_{0 i} \nabla_{\lambda} \hat{g}_{00} \\
& \nabla_{\lambda} \hat{g}_{i j}=:\left(\nabla_{\lambda} g\right)\left(b_{i}, b_{j}\right)=\nabla_{\lambda} g_{i j}+\left(\alpha^{0}\right)^{2}\left(\breve{g}_{0 i} \nabla_{\lambda} \breve{g}_{0 j}+\breve{g}_{0 j} \nabla_{\lambda} \breve{g}_{0 i}\right)+\left(\alpha^{0}\right)^{4} \breve{g}_{0 i} \breve{g}_{0 j} \nabla_{\lambda} \hat{g}_{00} \\
& \nabla_{\lambda} \hat{g}^{00}=:\left(\nabla_{\lambda} \bar{g}\right)\left(\beta^{0}, \beta^{0}\right)=-\nabla_{\lambda}\left(\alpha^{0}\right)^{2}=\left(\alpha^{0}\right)^{4} \breve{g}_{0 \nu} \breve{g}_{0 \mu} \nabla_{\lambda} g^{\nu \mu} \\
& =\left(\alpha^{0}\right)^{4}\left(\breve{g}_{0 \nu} \breve{g}_{0 \mu} \partial_{\lambda} g^{\nu \mu}-2 \breve{g}_{0 \sigma} \breve{\delta}_{0}^{\rho} K_{\lambda}{ }^{\sigma} \rho\right) \\
& \nabla_{\lambda} \hat{g}^{i 0}=:\left(\nabla_{\lambda} \bar{g}\right)\left(\beta^{i}, \beta^{0}\right)=-\left(\alpha^{0}\right)^{2} \breve{g}_{0 \mu} \nabla_{\lambda} \breve{g}^{i \mu}=-\left(\alpha^{0}\right)^{2}\left(\breve{g}_{0 \mu} \partial_{\lambda} \breve{g}^{i \mu}-\left(\breve{\delta}_{0}^{\rho} \breve{\delta}_{\sigma}^{i}+\breve{g}^{i \rho} \breve{g}_{0 \sigma} K_{\lambda}{ }^{\sigma} \rho\right),\right. \\
& \nabla_{\lambda} \hat{g}^{i j}=:\left(\nabla_{\lambda} \bar{g}\right)\left(\beta^{i}, \beta^{j}\right)=\breve{\delta}_{\nu}^{i} \breve{\delta}_{\mu}^{j} \nabla_{\lambda} g^{\nu \mu}=\partial_{\lambda} \hat{g}^{i j}-\left(\breve{g}^{j \rho} \breve{\delta}_{\sigma}^{i}+\breve{g}^{i \rho} \breve{\delta}_{\sigma}^{j}\right) K_{\lambda}{ }^{\sigma}{ }_{\rho} .
\end{aligned}
$$

Proof. We have

$$
\begin{aligned}
\nabla_{\lambda} g= & \nabla_{\lambda} g_{\nu \mu} d^{\nu} \otimes d^{\mu} \\
= & \nabla_{\lambda} g_{\nu \mu}\left(\breve{\delta}_{0}^{\nu} \beta^{0}+\left(\delta_{i}^{\nu}+\left(\alpha^{0}\right)^{2} \breve{g}_{0 i} \breve{\delta}_{0}^{\nu}\right) \beta^{i}\right) \otimes\left(\breve{\delta}_{0}^{\mu} \beta^{0}+\left(\delta_{j}^{\mu}+\left(\alpha^{0}\right)^{2} \breve{g}_{0 j} \breve{\delta}_{0}^{\mu}\right) \beta^{j}\right) \\
= & \nabla_{\lambda} g_{\nu \mu}\left[\breve{\delta}_{0}^{\nu} \breve{\delta}_{0}^{\mu} \beta^{0} \otimes \beta^{0}+\breve{\delta}_{0}^{\nu}\left(\delta_{j}^{\mu}+\left(\alpha^{0}\right)^{2} \breve{g}_{0 j} \breve{\delta}_{0}^{\mu}\right) \beta^{0} \otimes \beta^{j}\right. \\
& \left.\left.+\left(\delta_{i}^{\nu}+\left(\alpha^{0}\right)^{2} \breve{g}_{0 i} \breve{\delta}_{0}^{\nu}\right) \breve{\delta}_{0}^{\mu} \beta^{i} \otimes \beta^{0}+\left(\delta_{i}^{\nu}+\left(\alpha^{0}\right)^{2} \breve{g}_{0 i} \breve{\delta}_{0}^{\nu}\right)\left(\delta_{j}^{\mu}+\left(\alpha^{0}\right)^{2} \breve{g}_{0 j} \breve{\delta}_{0}^{\mu}\right)\right) \beta^{i} \otimes \beta^{j}\right] .
\end{aligned}
$$

Then, we obtain

$$
\begin{aligned}
& \nabla_{\lambda} g\left(b_{0}, b_{0}\right)=\breve{\delta}_{0}^{\nu} \breve{\delta}_{0}^{\mu} \nabla_{\lambda} g_{\nu \mu}, \\
& \nabla_{\lambda} g\left(b_{i}, b_{0}\right)=\left(\delta_{i}^{\nu} \breve{\delta}_{0}^{\mu}+\left(\alpha^{0}\right)^{2} \breve{g}_{0 i} \breve{\delta}_{0}^{\nu} \breve{\delta}_{0}^{\mu}\right) \nabla_{\lambda} g_{\nu \mu}, \\
& \nabla_{\lambda} g\left(b_{i}, b_{j}\right)=\left(\delta_{i}^{\nu} \breve{\delta}_{j}^{\mu}+\left(\alpha^{0}\right)^{2}\left(\breve{g}_{0 i} \breve{\delta}_{0}^{\nu} \delta_{j}^{\mu}+\breve{g}_{0 j} \breve{\delta}_{0}^{\mu} \delta_{i}^{\nu}\right)+\left(\alpha^{0}\right)^{4} \breve{g}_{0 i} \breve{g}_{0 j} \breve{\delta}_{0}^{\nu} \breve{\delta}_{0}^{\mu}\right) \nabla_{\lambda} g_{\nu \mu} .
\end{aligned}
$$

Analogously, we have

$$
\begin{aligned}
\nabla_{\lambda} \bar{g}= & \nabla_{\lambda} g^{\nu \mu} \partial_{\nu} \otimes \partial_{\mu} \\
= & \nabla_{\lambda} g^{\nu \mu}\left(\breve{\delta}_{\nu}^{i} b_{i}-\left(\alpha^{0}\right)^{2} \breve{g}_{0 \nu} b_{0}\right) \otimes\left(\breve{\delta}_{\mu}^{j} b_{j}-\left(\alpha^{0}\right)^{2} \breve{g}_{0 \mu} b_{0}\right) \\
= & \nabla_{\lambda} g^{\nu \mu}\left[\breve{\delta}_{\nu}^{i} \breve{\delta}_{\mu}^{j} b_{i} \otimes b_{j}-\left(\alpha^{0}\right)^{2}\left(\breve{g}_{0 \nu} \breve{\delta}_{\mu}^{j} b_{0} \otimes b_{j}+\breve{g}_{0 \mu} \breve{\delta}_{\nu}^{i} b_{i} \otimes b_{0}\right)\right. \\
& \left.+\left(\alpha^{0}\right)^{4} \breve{g}_{0 \nu} \breve{g}_{0 \mu} b_{0} \otimes b_{0}\right] .
\end{aligned}
$$

Then, we obtain

$$
\begin{aligned}
\nabla_{\lambda} \bar{g}\left(\beta^{0}, \beta^{0}\right) & =\left(\alpha^{0}\right)^{4} \breve{g}_{0 \nu} \breve{g}_{0 \mu} \nabla_{\lambda} g^{\nu \mu}, \quad \nabla_{\lambda} \bar{g}\left(\beta^{i}, \beta^{0}\right)=-\left(\alpha^{0}\right)^{2} \breve{g}_{0 \mu} \breve{\delta}_{\nu}^{i} \nabla_{\lambda} g^{\nu \mu}, \\
\nabla_{\lambda} \bar{g}\left(\beta^{i}, \beta^{j}\right) & =\breve{\delta}_{\nu}^{i} \breve{\delta}_{\mu}^{j} \nabla_{\lambda} g^{\nu \mu} .
\end{aligned}
$$


6.3. Lemma. We have the following coordinate expressions

$$
\begin{aligned}
& d_{K} g=2\left(\nabla_{\lambda} \breve{g}_{0 \mu}-\breve{g}_{0 \sigma} K_{\lambda}{ }^{\sigma}{ }_{\mu}\right)\left(d^{\lambda} \wedge d^{\mu}\right) \otimes \beta^{0}
\end{aligned}
$$

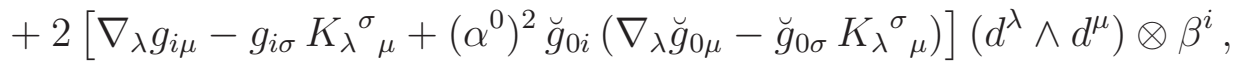

$$
\begin{aligned}
& g \otimes d_{K} g=2 \hat{g}_{00}\left(\nabla_{\lambda} \breve{g}_{0 \mu}-\breve{g}_{0 \sigma} K_{\lambda}{ }^{\sigma}{ }_{\mu}\right)\left(d^{\lambda} \wedge d^{\mu}\right) \otimes\left(\beta^{0} \otimes \beta^{0} \otimes \beta^{0}\right) \\
& +2 \hat{g}_{i j}\left(\nabla_{\lambda} \breve{g}_{0 \mu}-\breve{g}_{0 \sigma} K_{\lambda}{ }^{\sigma}{ }_{\mu}\right)\left(d^{\lambda} \wedge d^{\mu}\right) \otimes\left(\beta^{i} \otimes \beta^{j} \otimes \beta^{0}\right) \\
& +2 \hat{g}_{00}\left[\nabla_{\lambda} g_{i \mu}-g_{i \sigma} K_{\lambda}{ }^{\sigma}{ }_{\mu}+\left(\alpha^{0}\right)^{2} \breve{g}_{0 i}\left(\nabla_{\lambda} \breve{g}_{0 \mu}-\breve{g}_{0 \sigma} K_{\lambda}{ }^{\sigma}{ }_{\mu}\right)\right] \\
& \left(d^{\lambda} \wedge d^{\mu}\right) \otimes\left(\beta^{0} \otimes \beta^{0} \otimes \beta^{i}\right) \\
& +2 \hat{g}_{i j}\left[\nabla_{\lambda} g_{k \mu}-g_{k \sigma} K_{\lambda}{ }^{\sigma}{ }_{\mu}+\left(\alpha^{0}\right)^{2} \breve{g}_{0 k}\left(\nabla_{\lambda} \breve{g}_{0 \mu}-\breve{g}_{0 \sigma} K_{\lambda}{ }^{\sigma}{ }_{\mu}\right)\right] \\
& \left(d^{\lambda} \wedge d^{\mu}\right) \otimes\left(\beta^{i} \otimes \beta^{j} \otimes \beta^{k}\right) \text {. }
\end{aligned}
$$

Proof. We have

$$
\begin{aligned}
& d_{K} g=2\left(\nabla_{\lambda} g_{\rho \mu}-g_{\sigma \rho} K_{\lambda}{ }^{\sigma}{ }_{\mu}\right)\left(d^{\lambda} \wedge d^{\mu}\right) \otimes d^{\rho} \\
& =2\left(\nabla_{\lambda} \breve{g}_{0 \mu}-\breve{g}_{0 \sigma} K_{\lambda}{ }^{\sigma}{ }_{\mu}\right)\left(d^{\lambda} \wedge d^{\mu}\right) \otimes \beta^{0} \\
& +2\left[\nabla_{\lambda} g_{i \mu}-g_{i \sigma} K_{\lambda}{ }^{\sigma}{ }_{\mu}+\left(\alpha^{0}\right)^{2} \breve{g}_{0 i}\left(\nabla_{\lambda} \breve{g}_{0 \mu}-\breve{g}_{0 \sigma} K_{\lambda}{ }^{\sigma}{ }_{\mu}\right)\right]\left(d^{\lambda} \wedge d^{\mu}\right) \otimes \beta^{i} .
\end{aligned}
$$

We can write

$$
g=g_{\lambda \mu} d^{\lambda} \otimes d^{\mu}=\hat{g}_{00} \beta^{0} \otimes \beta^{0}+\hat{g}_{i j} \beta^{i} \otimes \beta^{j}
$$

hence

$$
\begin{aligned}
& g \otimes d_{K} g=2 g_{\rho \sigma}\left(\nabla_{\lambda} g_{\tau \mu}-g_{\omega \tau} K_{\lambda}{ }^{\omega}{ }_{\mu}\right)\left(d^{\lambda} \wedge d^{\mu}\right) \otimes\left(d^{\rho} \otimes d^{\sigma} \otimes d^{\tau}\right) \\
& =2 \hat{g}_{00}\left(\nabla_{\lambda} \breve{g}_{0 \mu}-\breve{g}_{0 \sigma} K_{\lambda}{ }^{\sigma}{ }_{\mu}\right)\left(d^{\lambda} \wedge d^{\mu}\right) \otimes\left(\beta^{0} \otimes \beta^{0} \otimes \beta^{0}\right) \\
& +2 \hat{g}_{i j}\left(\nabla_{\lambda} \breve{g}_{0 \mu}-\breve{g}_{0 \sigma} K_{\lambda}{ }^{\sigma}{ }_{\mu}\right)\left(d^{\lambda} \wedge d^{\mu}\right) \otimes\left(\beta^{i} \otimes \beta^{j} \otimes \beta^{0}\right) \\
& +2 \hat{g}_{00}\left[\nabla_{\lambda} g_{i \mu}-g_{i \sigma} K_{\lambda}{ }^{\sigma}{ }_{\mu}+\left(\alpha^{0}\right)^{2} \breve{g}_{0 i}\left(\nabla_{\lambda} \breve{g}_{0 \mu}-\breve{g}_{0 \sigma} K_{\lambda}{ }^{\sigma}{ }_{\mu}\right)\right] \\
& \left(d^{\lambda} \wedge d^{\mu}\right) \otimes\left(\beta^{0} \otimes \beta^{0} \otimes \beta^{i}\right) \\
& +2 \hat{g}_{i j}\left[\nabla_{\lambda} g_{k \mu}-g_{k \sigma} K_{\lambda}{ }^{\sigma}{ }_{\mu}+\left(\alpha^{0}\right)^{2} \breve{g}_{0 k}\left(\nabla_{\lambda} \breve{g}_{0 \mu}-\breve{g}_{0 \sigma} K_{\lambda}{ }^{\sigma}{ }_{\mu}\right)\right] \\
& \left(d^{\lambda} \wedge d^{\mu}\right) \otimes\left(\beta^{i} \otimes \beta^{j} \otimes \beta^{k}\right) \text {. }
\end{aligned}
$$

6.4. Lemma. For each $X, Y \in \sec (\boldsymbol{E}, T \boldsymbol{E})$, we have

$$
d_{K} g(X, Y)(\text { д })=2\left(\text { д }^{*} L_{\tilde{K}} \tilde{g}^{b}\right)(X, Y),
$$

where $\tilde{g}^{b}=$ id $\otimes g^{b}: \mathbb{T}^{*} \otimes T \boldsymbol{E} \rightarrow \mathbb{T}^{*} \otimes\left(\mathbb{L}^{2} \otimes T^{*} \boldsymbol{E}\right)$.

Proof. The claim follows from the coordinate expressions

$$
\begin{aligned}
d_{K} g(X, Y)(\text { д }) & =c \alpha^{0}\left(\partial_{\lambda} g_{\mu \rho}+g_{\mu \sigma} K_{\lambda}{ }^{\sigma}{ }_{\rho}-\partial_{\mu} g_{\lambda \rho}-g_{\lambda \sigma} K_{\mu}{ }^{\sigma}{ }_{\rho}\right) \breve{\delta}_{0}^{\rho} X^{\lambda} Y^{\mu}, \\
\text { д }^{*} L_{\tilde{K}} \tilde{g}^{b} & =c \alpha^{0}\left(\partial_{\lambda} g_{\mu \rho}+g_{\mu \sigma} K_{\lambda}{ }^{\sigma}{ }_{\rho}\right) \breve{\delta}_{0}^{\rho} d^{\lambda} \wedge d^{\mu} .
\end{aligned}
$$


6.5. Lemma. да $\widetilde{\Upsilon}_{\|}$is a horizontal 2-form and, for each $X, Y \in \sec (\boldsymbol{E}, T \boldsymbol{E})$, we have

$$
\text { д }{ }^{*} \widetilde{\Upsilon}_{\|}(X, Y)=\frac{1}{4 c^{2}}\left(g(\text { д, } Y)\left(\nabla_{X} g\right)\left(\text { д, д) }-g(\text { д, } X)\left(\nabla_{Y} g\right)(\text { д, д })\right)\right. \text {. }
$$

Proof. The coordinate expression of $д^{*} \widetilde{\Upsilon}_{\|}$from Proposition 4.11 can be rewritten as

$$
\text { , } \text { д }^{*} \widetilde{\Upsilon}_{\|}=\frac{1}{2} c\left(\alpha^{0}\right)^{3} \breve{g}_{0 \mu} \nabla_{\lambda} \hat{g}_{00} d^{\lambda} \wedge d^{\mu} .
$$

Then, we obtain

$$
\begin{aligned}
\text { д* } \widetilde{\Upsilon}_{\|}(X, Y) & =\frac{1}{4} c\left(\alpha^{0}\right)^{3}\left(\breve{g}_{0 \mu} \nabla_{\lambda} \hat{g}_{00}-\breve{g}_{0 \lambda} \nabla_{\mu} \hat{g}_{00}\right) X^{\lambda} Y^{\mu} \\
& =\frac{1}{4 c^{2}}\left(g(\text { д, } Y)\left(\nabla_{X} g\right)\left(\text { д, д) }-g(\text { д, } X)\left(\nabla_{Y} g\right)(\text { д, д })\right) .\right.
\end{aligned}
$$

6.6. Lemma. We have the coordinate expressions

$$
\begin{aligned}
L_{\chi(K)} \tau= & \frac{\alpha^{0}}{c}\left(\nabla_{\mu} \breve{g}_{0 \lambda}-\breve{g}_{0 \rho} K_{\mu}{ }^{\rho}{ }_{\lambda}+\frac{1}{2}\left(\alpha^{0}\right)^{2} \breve{g}_{0 \lambda} \nabla_{\mu} \hat{g}_{00}\right) d^{\lambda} \wedge d^{\mu}, \\
L_{R[\chi(K)]} \tau= & -\frac{\alpha^{0}}{c} \breve{g}_{\lambda \rho} R[K]_{\mu \nu}{ }^{\rho}{ }_{\sigma} \breve{\delta}_{0}^{\sigma} d^{\lambda} \wedge d^{\mu} \wedge d^{\nu}, \\
L_{\nu_{\tau}(X)} L_{\chi(K)} \tau= & \frac{1}{c^{2}}\left[\nabla_{\mu} g_{i \lambda}-g_{i \rho} K_{\mu}{ }^{\rho}{ }_{\lambda}\right. \\
& +\left(\alpha^{0}\right)^{2}\left(\breve{g}_{0 i}\left(\nabla_{\mu} \breve{g}_{0 \lambda}-\breve{g}_{0 \rho} K_{\mu}{ }^{\rho}{ }_{\lambda}\right)+\breve{g}_{0 \lambda} \nabla_{\mu} \breve{g}_{0 i}+\frac{1}{2} \breve{g}_{i \lambda} \nabla_{\mu} \hat{g}_{00}\right) \\
& \left.+\left(\alpha^{0}\right)^{4} \breve{g}_{0 i} \breve{g}_{0 \lambda} \nabla_{\mu} \hat{g}_{00}\right] \tilde{X}^{i} d^{\lambda} \wedge d^{\mu} .
\end{aligned}
$$

Proof. The above equalities follow from (5.14), (5.15) and (5.16), by taking into account the equality $\Gamma=\chi(K)$ and the identities $g_{p \mu} \breve{\delta}_{\nu}^{p}=g_{\nu \mu}-\breve{g}_{0 \mu} \delta_{\nu}^{0}, \breve{g}_{0 p} \breve{\delta}_{\nu}^{p}=\breve{g}_{0 \nu}-\hat{g}_{00} \delta_{\nu}^{0}$, and $\hat{g}_{i p} \breve{\delta}_{\nu}^{p}=\breve{g}_{i \nu}$.

6.7. Lemma. For each $X, Y \in \sec (\boldsymbol{E}, T \boldsymbol{E})$, we have

$$
\begin{aligned}
L_{\chi(K)} & \tau(X, Y)=\frac{1}{c^{2}}\left(\text { д }^{*} L_{\tilde{K}} \tilde{g}^{b}\right)(X, Y)-\frac{1}{c^{2}} \text { д }^{*} \widetilde{\Upsilon}_{\|}(X, Y) \\
& =-\frac{1}{2 c^{2}} d_{K} g(X, Y)\left(\text { д) }+\frac{1}{4 c^{4}}\left(g(\text { д, } X)\left(\nabla_{Y} g\right)(\text { д, д })-g(\text { д, } Y)\left(\nabla_{X} g\right)(\text { д, , д) }) .\right.\right.
\end{aligned}
$$

Proof. It follows from the coordinate expressions (6.1), (6.3) and Lemmas 6.4 and 6.5,

6.8. Lemma. $L_{\chi(K)} \tau=0$ if and only if, for each $X, Y \in \sec (\boldsymbol{E}, T \boldsymbol{E})$,

$$
g\left(b_{0}, b_{0}\right) d_{K} g(X, Y)\left(b_{0}\right)+\frac{1}{2} g\left(b_{0}, X\right)\left(\nabla_{Y} g\right)\left(b_{0}, b_{0}\right)-\frac{1}{2} g\left(b_{0}, Y\right)\left(\nabla_{X} g\right)\left(b_{0}, b_{0}\right)=0 .
$$

Proof. In virtue of the above Lemma 6.7, we have $L_{\chi(K)} \tau=0$ if and only if

$$
-\frac{1}{2 c^{2}} d_{K} g(X, Y)\left(\text { д) }+\frac{1}{4 c^{4}}\left(g ( \text { д, } X ) ( \nabla _ { Y } g ) \left(\text { д, д) }-g(\text { д, } Y)\left(\nabla_{X} g\right)(\text { д, д) })=0 .\right.\right.\right.
$$


Moreover, by multiplying the above equality by $2 c^{4}$ and by taking into account the fact that $g\left(\right.$ д, д) $=-c^{2}$, the above equality is equivalent to

$$
g\left(\text { д, д) } d_{K} g(X, Y)(\text { д })+\frac{1}{2}\left(g ( \text { д, } X ) ( \nabla _ { Y } g ) \left(\text { д, д) }-g(\text { д, } Y)\left(\nabla_{X} g\right)(\text { д, д) })=0,\right.\right.\right.
$$

and, by recalling the coordinate expression д $=c \alpha^{0} b_{0}$, we obtain (6.6).

6.9. Lemma. $L_{\chi(K)} \tau=0$ implies

$$
\begin{aligned}
g\left(b_{0}, b_{0}\right) & d_{K} g(X, Y)\left(b_{i}\right)+2 g\left(b_{0}, b_{i}\right) d_{K} g(X, Y)\left(b_{0}\right) \\
& +g\left(b_{0}, X\right)\left(\nabla_{Y} g\right)\left(b_{0}, b_{i}\right)-g\left(b_{0}, Y\right)\left(\nabla_{X} g\right)\left(b_{0}, b_{i}\right) \\
& +\frac{1}{2} g\left(b_{i}, X\right)\left(\nabla_{Y} g\right)\left(b_{0}, b_{0}\right)-\frac{1}{2} g\left(b_{i}, Y\right)\left(\nabla_{X} g\right)\left(b_{0}, b_{0}\right)=0, \\
2 g\left(b_{i}, b_{j}\right) & d_{K} g(X, Y)\left(b_{0}\right)+2 g\left(b_{0}, b_{i}\right) d_{K} g(X, Y)\left(b_{j}\right)+2 g\left(b_{0}, b_{j}\right) d_{K} g(X, Y)\left(b_{i}\right) \\
& +g\left(b_{i}, X\right)\left(\nabla_{Y} g\right)\left(b_{0}, b_{j}\right)+g\left(b_{j}, X\right)\left(\nabla_{Y} g\right)\left(b_{0}, b_{i}\right) \\
& -g\left(b_{i}, Y\right)\left(\nabla_{X} g\right)\left(b_{0}, b_{j}\right)-g\left(b_{j}, Y\right)\left(\nabla_{X} g\right)\left(b_{0}, b_{i}\right) \\
& +g\left(b_{0}, X\right)\left(\nabla_{Y} g\right)\left(b_{i}, b_{j}\right)-g\left(b_{0}, Y\right)\left(\nabla_{X} g\right)\left(b_{i}, b_{j}\right)=0, \\
2 g\left(b_{i}, b_{j}\right) & d_{K} g(X, Y)\left(b_{k}\right)+2 g\left(b_{k}, b_{i}\right) d_{K} g(X, Y)\left(b_{j}\right)+2 g\left(b_{k}, b_{j}\right) d_{K} g(X, Y)\left(b_{i}\right) \\
& +g\left(b_{i}, X\right)\left(\nabla_{Y} g\right)\left(b_{k}, b_{j}\right)+g\left(b_{j}, X\right)\left(\nabla_{Y} g\right)\left(b_{k}, b_{i}\right) \\
& -g\left(b_{i}, Y\right)\left(\nabla_{X} g\right)\left(b_{k}, b_{j}\right)-g\left(b_{j}, Y\right)\left(\nabla_{X} g\right)\left(b_{k}, b_{i}\right) \\
& +g\left(b_{k}, X\right)\left(\nabla_{Y} g\right)\left(b_{i}, b_{j}\right)-g\left(b_{k}, Y\right)\left(\nabla_{X} g\right)\left(b_{i}, b_{j}\right)=0 .
\end{aligned}
$$

Proof. $L_{\chi(K)} \tau=0$ implies $L_{\nu_{\tau}(Z)}^{r} L_{\chi(K)} \tau=0$, for each $Z \in \sec (\boldsymbol{E}, T \boldsymbol{E})$ and for each integer $r \geq 1$. Moreover, $L_{\nu_{\tau}(Z)}^{r} L_{\chi(K)} \tau$ is a horizontal 2-form and, for each, $X, Y \in$ $\sec (\boldsymbol{E}, T \boldsymbol{E})$, we have $\left(L_{\nu_{\tau}(Z)}^{r} L_{\chi(K)} \tau\right)(X, Y)=\nu_{\tau}(Z)^{r} \cdot L_{\chi(K)} \tau(X, Y)$. Let us denote the left hand side of (6.6) by $K(X, Y)$.

Then, from Lemma 6.7.

$$
\begin{aligned}
\nu_{\tau}(Z)^{r} \cdot L_{\chi(K)} \tau(X, Y) & =\frac{1}{2 c^{2}} \nu_{\tau}(Z)^{r} \cdot\left(\left(c \alpha^{0}\right)^{3} K(X, Y)\right) \\
& =\frac{c}{2} \sum_{k=0}^{r}\left(\nu_{\tau}(Z)^{r-k} \cdot\left(\alpha^{0}\right)^{3}\right)\left(\nu_{\tau}(Z)^{k} \cdot K(X, Y)\right) .
\end{aligned}
$$

Now, $L_{\chi(K)} \tau=0$ and Lemma 6.7 imply $\nu_{\tau}(Z)^{k} . K(X, Y)=0, k=1, \ldots, r$.

For $k=1$, we have

$$
\begin{aligned}
\nu_{\tau}(Z) . K(X, Y)= & \frac{1}{c \alpha^{0}} \tilde{Z}^{i} \partial_{i}^{0} \cdot K(X, Y) \\
= & \frac{1}{c \alpha^{0}} \tilde{Z}^{i}\left[g\left(b_{0}, b_{0}\right) d_{K} g(X, Y)\left(\partial_{i}\right)+2 g\left(b_{0}, \partial_{i}\right) d_{K} g(X, Y)\left(b_{0}\right)\right. \\
& +g\left(b_{0}, X\right)\left(\nabla_{Y} g\right)\left(b_{0}, \partial_{i}\right)-g\left(b_{0}, Y\right)\left(\nabla_{X} g\right)\left(b_{0}, \partial_{i}\right) \\
& \left.+\frac{1}{2} g\left(\partial_{i}, X\right)\left(\nabla_{Y} g\right)\left(b_{0}, b_{0}\right)-\frac{1}{2} g\left(\partial_{i}, Y\right)\left(\nabla_{X} g\right)\left(b_{0}, b_{0}\right)\right] \\
= & \frac{1}{c \alpha^{0}} \tilde{Z}^{i}\left[g\left(b_{0}, b_{0}\right) d_{K} g(X, Y)\left(b_{i}\right)+2 g\left(b_{0}, b_{i}\right) d_{K} g(X, Y)\left(b_{0}\right)\right.
\end{aligned}
$$




$$
\begin{aligned}
& +g\left(b_{0}, X\right)\left(\nabla_{Y} g\right)\left(b_{0}, b_{i}\right)-g\left(b_{0}, Y\right)\left(\nabla_{X} g\right)\left(b_{0}, b_{i}\right) \\
& +\frac{1}{2} g\left(b_{i}, X\right)\left(\nabla_{Y} g\right)\left(b_{0}, b_{0}\right)-\frac{1}{2} g\left(b_{i}, Y\right)\left(\nabla_{X} g\right)\left(b_{0}, b_{0}\right) \\
& \left.-3\left(\alpha^{0}\right)^{2} \breve{g}_{0 i} K(X, Y)\right]
\end{aligned}
$$

which vanishes if and only if (6.7) is satisfied.

Similarly, the condition $\nu_{\tau}(Z)^{2} \cdot K(X, Y)=0$ implies (6.8) and finally the condition $\nu_{\tau}(Z)^{3} \cdot K(X, Y)=0$ implies (6.9).

6.10. Theorem. $L_{\chi(K)} \tau=0$ if and only if the following condition hold, for each $X, Y, Z \in \sec (\boldsymbol{E}, T \boldsymbol{E})$,

$$
g(Z, Z) d_{K} g(X, Y)(Z)+\frac{1}{2} g(Z, Y)\left(\nabla_{X} g\right)(Z, Z)-\frac{1}{2} g(Z, X)\left(\nabla_{Y} g\right)(Z, Z)=0 .
$$

Proof. In virtue of Lemma 6.7, the condition (6.10) implies $L_{\chi(K)} \tau=0$.

On the other hand, in an orthogonal adapted base, we have the coordinate expression $Z=\tilde{Z}^{0} b_{0}+\tilde{Z}^{i} b_{i}$. If $L_{\chi(K)} \tau=0$, then, by linearity and in virtue of the above Lemmas 6.8 and 6.9, the condition (6.10) is satisfied.

6.11. Corollary. If additionally $K$ is a torsion free linear spacetime connection such that $\nabla g$ and $g \otimes \nabla g$ are symmetric, then $L_{\chi(K)} \tau=0$.

Proof. For a torsion free connection $K$ the symmetry of $\nabla g$ is equivalent to $d_{K} g=0$. Then, the conditions $\nabla g$ and $g \otimes \nabla g$ being symmetric imply (6.10) and this is equivalent to $L_{\chi(K)} \tau=0$.

6.2. Almost-cosymplectic-contact structure. Let us analyze conditions for $\left(-c^{2} \tau, \Omega\right)$ to be a scaled almost-cosymplectic-contact structure.

6.12. Lemma. We have the coordinate expression

$$
\begin{aligned}
d \Omega= & -c \alpha^{0}\left[\left(\alpha^{0}\right)^{4} \breve{g}_{0 i} \breve{g}_{0 \mu} \nabla_{\lambda} \hat{g}_{00}\right. \\
& +\left(\alpha^{0}\right)^{2}\left(\frac{1}{2} \breve{g}_{i \mu} \nabla_{\lambda} \hat{g}_{00}+\breve{g}_{0 \mu} \nabla_{\lambda} \breve{g}_{0 i}+\breve{g}_{0 i}\left(\nabla_{\lambda} \breve{g}_{0 \mu}-\breve{g}_{0 \rho} K_{\lambda}{ }^{\rho}{ }_{\mu}\right)\right) \\
& \left.+\nabla_{\lambda} g_{i \mu}-g_{i \rho} K_{\lambda}{ }^{\rho}{ }_{\mu}\right]\left(d_{0}^{i}-\breve{\delta}_{\tau}^{i} K_{\nu}{ }^{\rho}{ }_{\sigma} \breve{\delta}_{0}^{\sigma} d^{\nu}\right) \wedge d^{\lambda} \wedge d^{\mu} \\
& +\frac{1}{2} c \alpha^{0} \breve{g}_{\mu \rho} R[K]_{\beta \lambda}{ }^{\rho}{ }_{\sigma} \breve{\delta}_{0}^{\sigma} d^{\beta} \wedge d^{\lambda} \wedge d^{\mu} .
\end{aligned}
$$

Proof. The identity $\breve{g}_{p \mu} \breve{\delta}_{\nu}^{p}=\breve{g}_{\nu \mu}$ and (5.7) yield

$$
\begin{aligned}
d \Omega= & -c\left(\partial_{\lambda}\left(\alpha^{0} \breve{g}_{i \mu}\right)+\partial_{i}^{0}\left(\alpha^{0} \breve{g}_{p \mu} \breve{\delta}_{\nu}^{p} K_{\lambda}{ }^{\nu}{ }_{\rho} \breve{\delta}_{0}^{\rho}\right)\right) d_{0}^{i} \wedge d^{\lambda} \wedge d^{\mu} \\
& -c \partial_{\beta}\left(\alpha^{0} \breve{g}_{p \mu} \breve{\delta}_{\nu}^{p} K_{\lambda}{ }^{\nu}{ }_{\rho} \breve{\delta}_{0}^{\rho}\right) d^{\beta} \wedge d^{\lambda} \wedge d^{\mu} \\
= & -c \alpha^{0}\left[\frac{3}{2}\left(\alpha^{0}\right)^{4} \breve{g}_{0 i} \breve{g}_{0 \mu} \nabla_{\lambda} \hat{g}_{00}+\nabla_{\lambda} g_{i \mu}-g_{i \rho} K_{\lambda}{ }^{\rho}{ }_{\mu}\right. \\
& \left.+\left(\alpha^{0}\right)^{2}\left(\frac{1}{2} g_{i \mu} \nabla_{\lambda} \hat{g}_{00}+\breve{g}_{0 \mu} \nabla_{\lambda} \breve{g}_{0 i}+\breve{g}_{0 i}\left(\nabla_{\lambda} \breve{g}_{0 \mu}-\breve{g}_{0 \rho} K_{\lambda}{ }^{\rho}{ }_{\mu}\right)\right)\right] d_{0}^{i} \wedge d^{\lambda} \wedge d^{\mu} \\
= & -c \alpha^{0}\left[\frac{3}{2}\left(\alpha^{0}\right)^{4} \breve{g}_{0 \mu} \breve{g}_{0 \nu} K_{\lambda}{ }^{\nu}{ }_{\rho} \breve{\delta}_{0}^{\rho} \nabla_{\beta} \hat{g}_{00}+\left(\alpha^{0}\right)^{2}\left(\frac{1}{2} g_{\mu \nu} K_{\lambda}{ }_{\rho}{ }_{\rho} \breve{\delta}_{0}^{\rho} \nabla_{\beta} \hat{g}_{00}\right.\right. \\
& +\breve{g}_{0 \nu} K_{\lambda}{ }^{\nu}{ }_{\rho} \breve{\delta}_{0}^{\rho}\left(\nabla_{\beta} \breve{g}_{0 \mu}-\breve{g}_{0 \tau} K_{\beta}{ }^{\tau}{ }_{\mu}\right)+\breve{g}_{0 \mu} K_{\lambda}{ }^{\nu}{ }_{\rho} \breve{\delta}_{0}^{\rho}\left(\nabla_{\beta} \breve{g}_{0 \nu}-g_{\tau \nu} K_{\beta}{ }^{\tau} \breve{\delta}_{0}^{\sigma}\right)
\end{aligned}
$$




$$
\begin{aligned}
& \left.-\frac{1}{2} \breve{g}_{0 \mu} \breve{g}_{0 \nu} \breve{\delta}_{0}^{\rho} R[K]_{\beta \lambda}{ }^{\nu}{ }_{\rho}\right) \\
+ & \left.K_{\lambda}{ }^{\nu}{ }_{\rho} \breve{\delta}_{0}^{\rho}\left(\nabla_{\beta} g_{\mu \nu}-g_{\tau \nu} K_{\beta}{ }^{\tau}{ }_{\mu}\right)-\frac{1}{2} g_{\mu \nu} \breve{\delta}_{0}^{\rho} R[K]_{\beta \lambda}{ }^{\nu}{ }_{\rho}\right] d^{\beta} \wedge d^{\lambda} \wedge d^{\mu} .
\end{aligned}
$$

6.13. Theorem. The pair $\left(-c^{2} \tau, \Omega\right)$ is a scaled almost-cosymplectic-contact structure if and only if

$$
L_{\nu_{\tau}(X)} L_{\chi(K)} \tau=0, \quad \forall X \in \sec (\boldsymbol{E}, T \boldsymbol{E}), \quad \text { and } \quad L_{R[\chi(K)]} \tau=0 .
$$

Proof. It follows immediately from Theorem 5.19 by considering $\Gamma=\chi(K)$.

Eventually, we can prove directly Theorem by comparing the coordinate expression (6.11) with (6.4) and (6.5).

6.3. Almost-coPoisson-Jacobi structure. Let us analyze conditions for $\left(-\frac{1}{c^{2}} \gamma, \Lambda\right)$ to be a scaled contact structure.

6.14. Lemma. We have the coordinate expression

$$
\begin{aligned}
{[\gamma, \Lambda]=} & {\left[-\frac{1}{2}\left(\alpha^{0}\right)^{2} \breve{g}_{0 k}\left(\breve{g}^{j \lambda} \breve{g}^{k \rho}+\breve{g}^{j \rho} \breve{g}^{k \lambda}\right) \nabla_{\rho} \hat{g}_{00}+\left(\breve{g}^{j \sigma} \breve{\delta}_{0}^{\rho}-\breve{g}^{j \rho} \breve{\delta}_{0}^{\sigma}\right) K_{\rho}{ }^{\lambda} \sigma\right.} \\
& \left.+\breve{\delta}_{0}^{\rho} \nabla_{\rho} \breve{g}^{j \lambda}+\frac{1}{2}\left(g^{0 \rho} \breve{g}^{j \lambda}+g^{0 \lambda} \breve{g}^{j \rho}\right) \nabla_{\rho} \hat{g}_{00}\right]\left(\partial_{\lambda}+\breve{\delta}_{\nu}^{i} \breve{\delta}_{0}^{\kappa} K_{\lambda}{ }^{\nu}{ }_{\kappa} \partial_{i}^{0}\right) \wedge \partial_{j}^{0} \\
& +\breve{g}^{j \lambda} \breve{\delta}_{\nu}^{i} \breve{\delta}_{0}^{\rho} \breve{\delta}_{0}^{\sigma} R[K]_{\lambda \rho}{ }^{\nu}{ }_{\sigma} \partial_{i}^{0} \wedge \partial_{j}^{0}
\end{aligned}
$$

and, in the adapted base (5.1),

$$
\begin{aligned}
{[\gamma, \Lambda]=} & -\left(\alpha^{0}\right)^{2}\left[\frac{1}{2} \breve{g}^{j \rho}\left(\nabla_{\rho} \hat{g}_{00}-2 \breve{g}_{0 \nu} \breve{\delta}_{0}^{\sigma} K_{\rho}{ }^{\nu}{ }^{\prime}\right)\right. \\
& \left.+\breve{g}_{0 \lambda} \breve{\delta}_{0}^{\rho}\left(\nabla_{\rho} \breve{g}^{j \lambda}+\breve{g}^{j \sigma} K_{\rho}{ }^{\lambda}{ }_{\sigma}\right)\right] e_{0} \wedge e_{j}^{0} \\
- & {\left[\frac{1}{2}\left(\alpha^{0}\right)^{2} \hat{g}^{i j} \breve{\delta}_{0}^{\rho} \nabla_{\rho} \hat{g}_{00}+\breve{g}^{j \rho} \breve{\delta}_{\nu}^{i} \breve{\delta}_{0}^{\sigma} K_{\rho}{ }^{\nu}{ }_{\sigma}\right.} \\
& \left.-\breve{\delta}_{0}^{\rho}\left(\nabla_{\rho} \hat{g}^{j i}+\breve{\delta}_{\nu}^{i} \breve{g}^{j \sigma} K_{\rho}{ }^{\nu} \sigma+\hat{g}^{p i} \breve{\delta}_{0}^{\sigma} K_{\rho}{ }^{0} \sigma\right)\right] e_{i} \wedge e_{j}^{0} \\
- & \breve{g}^{j \lambda} \breve{\delta}_{\nu}^{i} \breve{\delta}_{0}^{\sigma} \breve{\delta}_{0}^{\rho} R[K]_{\lambda \rho}{ }^{\nu}{ }_{\sigma} e_{i}^{0} \wedge e_{j}^{0} .
\end{aligned}
$$

Proof. From Lemma 5.20 and the identity $\left(\alpha^{0}\right)^{2} \hat{g}_{00}=-1$ we get

$$
\begin{aligned}
{[\gamma, \Lambda]=} & \left(-\left(\alpha^{0}\right)^{2}\left(\breve{g}^{j \lambda} \breve{\delta}_{0}^{\rho}+\breve{g}^{j \rho} \breve{\delta}_{0}^{\lambda}\right)\left(\frac{1}{2} \partial_{\rho} \hat{g}_{00}+\breve{g}_{0 p} \breve{\delta}_{\nu}^{p} \breve{\delta}_{0}^{\sigma} K_{\rho}{ }^{\nu}\right)\right. \\
& +\breve{\delta}_{0}^{\rho} \partial_{\rho} \breve{g}^{j \lambda}-g^{0 \lambda} \breve{\delta}_{0}^{\rho} \breve{\delta}_{\nu}^{j} K_{\rho}{ }^{\nu}{ }_{\sigma} \breve{\delta}_{0}^{\sigma}-\delta_{p}^{\lambda} \breve{g}^{j \nu} \breve{\delta}_{\kappa}^{p} K_{\nu}{ }^{\kappa}{ }_{\sigma} \breve{\delta}_{0}^{\sigma}+\breve{g}^{j \lambda} \breve{\delta}_{0}^{\rho} K_{\rho}{ }^{0} \breve{\delta}_{0}^{\sigma} \\
& \left.-\breve{g}^{p \lambda} \breve{\delta}_{0}^{\rho} \breve{\delta}_{\nu}^{j} K_{\rho}{ }^{\nu} \sigma \delta_{p}^{\sigma}\right)\left(\partial_{\lambda}+\breve{\delta}_{\nu}^{i} \breve{\delta}_{0}^{\beta} K_{\lambda}{ }^{\nu}{ }_{\beta} \partial_{i}^{0}\right) \wedge \partial_{j}^{0} \\
& +\breve{g}^{j \lambda} \breve{\delta}_{\nu}^{i} \breve{\delta}_{0}^{\rho} \breve{\delta}_{0}^{\sigma} R[K]_{\lambda \rho}{ }^{\nu}{ }_{\sigma} \partial_{i}^{0} \wedge \partial_{j}^{0} \\
= & -\frac{1}{2}\left(\alpha^{0}\right)^{2}\left(\breve{g}^{j \lambda} \breve{\delta}_{0}^{\rho}+\breve{g}^{j \rho} \breve{\delta}_{0}^{\lambda}\right) \nabla_{\rho} \hat{g}_{00} \\
& \left.+\breve{\delta}_{0}^{\rho} \nabla_{\rho} \breve{g}^{j \lambda}+\left(\breve{g}^{j \sigma} \breve{\delta}_{0}^{\rho}-\breve{g}^{j \rho} \breve{\delta}_{0}^{\sigma}\right) K_{\rho}{ }^{\lambda}{ }_{\sigma}\right]\left(\partial_{\lambda}+\breve{\delta}_{\nu}^{i} \breve{\delta}_{0}^{\kappa} K_{\lambda}{ }^{\nu}{ }_{\kappa} \partial_{i}^{0}\right) \wedge \partial_{j}^{0} \\
& +\breve{g}^{j \lambda} \breve{\delta}_{\nu}^{i} \breve{\delta}_{0}^{\rho} \breve{\delta}_{0}^{\sigma} R[K]_{\lambda \rho}{ }^{\nu}{ }_{\sigma} \partial_{i}^{0} \wedge \partial_{j}^{0} .
\end{aligned}
$$

Finally, we obtain (6.13) by using the identity $\breve{\delta}_{0}^{\rho}=\breve{g}_{0 k} \breve{g}^{k \rho}-\frac{1}{\left(\alpha^{0}\right)^{2}} g^{0 \rho}$. 
6.15. Lemma. In the adapted base (5.1) we have the coordinate expression

$$
\begin{gathered}
{\left[-\frac{1}{c^{2}} \gamma, \Lambda\right]-\frac{1}{c^{2}} \gamma \wedge \Lambda^{\sharp}\left(L_{\gamma} \tau\right)=\frac{1}{c^{2}}\left[\frac{\left(\alpha^{0}\right)^{2}}{2 c^{2}} \hat{g}^{i j} \breve{\delta}_{0}^{\rho} \nabla_{\rho} \hat{g}_{00}+\breve{g}^{j \rho} \breve{\delta}_{\nu}^{i} \breve{\delta}_{0}^{\sigma} K_{\rho}{ }^{\nu} \sigma\right.} \\
\left.-\breve{\delta}_{0}^{\rho}\left(\nabla_{\rho} \hat{g}^{j i}+\breve{\delta}_{\nu}^{i} \breve{g}^{j \sigma} K_{\rho}{ }^{\nu}{ }_{\sigma}+\hat{g}^{p i} \breve{\delta}_{0}^{\sigma} K_{\rho}{ }^{0} \sigma\right)\right] e_{i} \wedge e_{j}^{0} \\
+\frac{1}{c^{2}} \breve{g}^{j \lambda} \breve{\delta}_{\nu}^{i} \breve{\delta}_{0}^{\sigma} \breve{\delta}_{0}^{\rho} R[K]_{\lambda \rho}{ }^{\nu}{ }_{\sigma} e_{i}^{0} \wedge e_{j}^{0} .
\end{gathered}
$$

6.16. Lemma. In the adapted base (5.1) we have the coordinate expression

$$
\begin{gathered}
{[\Lambda, \Lambda]-2 \gamma \wedge\left(\Lambda^{\sharp} \otimes \Lambda^{\sharp}\right)(d \tau)=} \\
=\frac{2}{c^{2}}\left[\left(\frac{1}{2} \hat{g}^{k i} \breve{g}^{j \rho} \nabla_{\rho} \hat{g}_{00}+\frac{1}{\left(\alpha^{0}\right)^{2}} \breve{g}^{k \rho}\left(\nabla_{\rho} \hat{g}^{k i}+\breve{\delta}_{\nu}^{i} \breve{g}^{j \sigma} K_{\rho}^{\nu}{ }^{\nu}+\hat{g}^{p i} \breve{\delta}_{0}^{\sigma} K_{\rho \sigma}{ }^{0}\right)\right) e_{i} \wedge e_{j}^{0} \wedge e_{k}^{0}\right. \\
\left.+\frac{1}{\left(\alpha^{0}\right)^{2}} \breve{g}^{i \rho} \breve{g}^{j \sigma} \breve{\delta}_{\nu}^{i} \breve{\delta}_{0}^{\sigma} R[K]_{\rho \sigma}{ }^{\nu}{ }_{\sigma} e_{i}^{0} \wedge e_{j}^{0} \wedge e_{k}^{0}\right] .
\end{gathered}
$$

6.17. Theorem. The pair $\left(-\frac{1}{c^{2}} \gamma, \Lambda\right)$ is a scaled almost-coPoisson-Jacobi pair if and only if the conditions (6.12) are satisfied.

Proof. It follows immediately from Theorem 5.31 by considering $\Gamma=\chi(K)$.

Eventually, we can prove directly Theorem by comparing the coordinate expressions (6.15) and (6.16) with

$$
{ }^{\sharp}\left(L_{R[\Gamma]} \tau\right)=-\frac{2 \hbar \alpha^{0}}{m c^{5}} \breve{g}^{k \lambda} \breve{\delta}_{0}^{\rho} \breve{\delta}_{\nu}^{i} \breve{\delta}_{0}^{\sigma} R_{\rho \lambda}{ }^{\nu}{ }_{\sigma} e_{i}^{0} \wedge e_{0} \wedge e_{k}^{0}-\frac{1}{c^{4}\left(\alpha^{0}\right)^{2}} \breve{g}^{j \lambda} \breve{g}^{k \mu} \breve{\delta}_{\nu}^{i} \breve{\delta}_{0}^{\sigma} R_{\lambda \mu}{ }^{\nu}{ } e_{i}^{0} \wedge e_{j}^{0} \wedge e_{k}^{0}
$$

and

$$
\begin{aligned}
\sharp\left(L_{\Lambda^{\sharp}(\omega)} L_{\chi(K)} \tau\right)= & -\frac{\hbar \alpha^{0}}{m c^{5}}\left[\frac{1}{2}\left(\alpha^{0}\right)^{2} \hat{g}^{j i} \breve{\delta}_{0}^{\rho} \nabla_{\rho} \hat{g}_{00}+\breve{g}^{j \rho} \breve{\delta}_{0}^{i} \breve{\delta}_{0}^{\sigma} K_{\rho}{ }^{\nu} \sigma\right. \\
& \left.-\breve{\delta}_{0}^{\rho}\left(\nabla_{\rho} \hat{g}^{j i}+\breve{\delta}_{\nu}^{i} \breve{g}^{j \sigma} K_{\rho}{ }^{\nu}+\hat{g}^{p i} \breve{\delta}_{0}^{\sigma} K_{\rho \sigma}{ }^{0}\right)\right] \widetilde{\omega}_{i} e_{0} \wedge e_{j}^{0} \\
- & \frac{1}{c^{4}\left(\alpha^{0}\right)^{2}}\left[\frac{1}{2}\left(\alpha^{0}\right)^{2} \hat{g}^{k i} \breve{g}^{j \rho} \nabla_{\rho} \hat{g}_{00}\right. \\
& \left.-\breve{g}^{j \rho}\left(\nabla_{\rho} \hat{g}^{k i}+\breve{\delta}_{\nu}^{i} \breve{g}^{j \sigma} K_{\rho}{ }^{\nu}{ }+\hat{g}^{p i} \breve{\delta}_{0}^{\sigma} K_{\rho}{ }^{0} \sigma\right)\right] \widetilde{\omega}_{i} e_{j}^{0} \wedge e_{k}^{0} .
\end{aligned}
$$

Then, $\left(-\frac{1}{c^{2}} \gamma, \Lambda\right)$ is a scaled almost-coPoisson-Jacobi structure if and only if

$$
\sharp\left(L_{\Lambda^{\sharp}(\omega)} L_{\Gamma} \tau\right)=0, \quad \text { for each } \omega \in \sec \left(\mathcal{J}_{1} \boldsymbol{E}, V_{\gamma}^{*} \mathcal{J}_{1} \boldsymbol{E}\right), \quad \text { and } \sharp\left(L_{R[\Gamma]} \tau\right)=0 \text {, }
$$

i.e. if and only if $L_{\nu_{\tau}(X)} L_{\Gamma} \tau=0$, for each $X \in \sec \left(\boldsymbol{E}, T \mathcal{J}_{1} \boldsymbol{E}\right)$, and $L_{R[\Gamma]} \tau=0$.

We can summarize the above results as follows.

6.18. Theorem. The following assertions are equivalent.

(1) $L_{\nu_{\tau}(X)} L_{\chi(K)} \tau=0$, for each $X \in \sec (\boldsymbol{E}, T \boldsymbol{E})$, and $L_{R[\chi(K)]} \tau=0$.

(2) $\Omega=0$, i.e. the pair $\left(-c^{2} \tau, \Omega\right)$ is a scaled almost-cosymplectic-contact pair;

(3) $\left[-\frac{1}{c^{2}} \gamma, \Lambda\right]=\frac{1}{c^{2}} \gamma \wedge \Lambda^{\sharp}\left(L_{\gamma} \tau\right),[\Lambda, \Lambda]=2 \gamma \wedge\left(\Lambda^{\sharp} \otimes \Lambda^{\sharp}\right)(d \tau)$, i.e. the pair $\left(-\frac{1}{c^{2}} \gamma, \Lambda\right)$ is a scaled almost-coPoisson-Jacobi pair. 
6.4. Contact structure. Let us analyse conditions for $\left(-c^{2} \tau, \Omega\right)$ to be a scaled contact structure.

6.19. Theorem. $\Omega=-c^{2} d \tau$, i.e. the pair $\left(-c^{2} \tau, \Omega\right)$ is a scaled contact structure, if and only if the identity (6.10) is satisfied.

Proof. It follows immediately from Theorems 5.35 and 6.10.

6.20. Corollary. In the particular case when $K$ is torsion free and the tensors $\nabla g$ and $g \otimes \nabla g$ are symmetric, the pair $\left(-c^{2} \tau, \Omega\right)$ is a scaled contact structure.

Proof. This follows from Theorem 5.35, Corollary 6.11 and Theorem 6.10.

Let us recall that, by Theorem 4.12 and Proposition 4.11 ,

$$
\Omega[g, K]=\text { д }^{*} \widetilde{\Upsilon}_{\perp}=\text { д }^{*} \widetilde{\Upsilon}-\text { д }^{*} \widetilde{\Upsilon}_{\|} \text {. }
$$

Then, we have the following result.

\subsection{Lemma.}

$$
\Omega=\text { д }^{*} \widetilde{\Upsilon}
$$

if and only if

$$
g(\text { д, } X) \nabla_{Y} g\left(\text { д, д) }=g(\text { д, } Y) \nabla_{X} g(\text { д, д) }, \quad \forall X, Y \in \sec (\boldsymbol{E}, T \boldsymbol{E}) .\right.
$$

Proof. The coordinate expression of $д^{*} \widetilde{\Upsilon}_{\|}$can be rewritten as

$$
\text { д }^{*} \widetilde{\Upsilon}_{\|}=\frac{1}{2} c\left(\alpha^{0}\right)^{3} \breve{g}_{0 \mu} \nabla_{\lambda} \hat{g}_{00} d^{\lambda} \wedge d^{\mu} .
$$

Then, $\Omega=$ д $^{*} \widetilde{\Upsilon}$ if and only if д $^{*} \widetilde{\Upsilon}_{\|}=0$, i.e., if and only if

$$
\breve{g}_{0 \mu} \nabla_{\lambda} \hat{g}_{00}-\breve{g}_{0 \lambda} \nabla_{\mu} \hat{g}_{00}=0,
$$

which is equivalent to

$$
g(\text { д, } X) \nabla_{Y} g\left(\text { д, д) }=g(\text { д, } Y) \nabla_{X} g(\text { д, д) } .\right.
$$

6.22. Remark. By Lemma 6.7, Theorems 6.10 and 6.19, the two conditions

$$
\begin{aligned}
d_{K} g & =0, \\
g(\text { д, } X) \nabla_{Y} g(\text { д, д }) & =g(\text { д, } Y) \nabla_{X} g(\text { д, д })
\end{aligned}
$$

imply that $\left(-c^{2} \tau, \Omega\right)$ is a scaled contact pair. But this fact can be viewed also as a consequence of Lemma 6.21 and Theorem 3.6. by considering the fact that $d_{K} g=0$ is equivalent to $\tilde{\Upsilon}=d \tilde{g}^{b}$, where $\tilde{g}^{b}=\mathrm{id} \otimes g^{b}$ is the Liouville 1 -form on $\tilde{T} \boldsymbol{E}$, and $\mathbf{д}^{*} d \tilde{g}^{b}=$ $-c^{2} \tau$.

6.23. Corollary. We have

$$
\text { д* } \widetilde{\Upsilon}-\text { д }^{*} \widetilde{\Upsilon}_{\|}=-c^{2}\left(d \tau+L_{\Gamma} \tau\right) .
$$

Proof. It follows from (6.17) and Corollary 5.34. 
6.5. Jacobi structure. Let us analyse conditions for $\left(-\frac{1}{c^{2}} \gamma, \Lambda\right)$ to define a scaled Jacobi structure.

6.24. Lemma. We have the coordinate expression

$$
\begin{aligned}
{[\Lambda, \Lambda]-\frac{2}{c^{2}} } & \gamma \wedge \Lambda=\frac{2}{c^{2}}\left[\frac{1}{2} \breve{g}^{j \rho} \breve{g}^{k \lambda} \nabla_{\rho} \hat{g}_{00}\right. \\
& \left.+\frac{1}{\left(\alpha^{0}\right)^{2}} \breve{g}^{k \rho}\left(\nabla_{\rho} \breve{g}^{j \lambda}+\breve{g}^{j \sigma} K_{\rho}{ }^{\lambda} \sigma\right)\right]\left(\partial_{\lambda}+\breve{\delta}_{\nu}^{i} K_{\lambda}{ }^{\nu}{ }_{\omega} \breve{\delta}_{0}^{\omega} \partial_{i}^{0}\right) \wedge \partial_{j}^{0} \wedge \partial_{k}^{0} \\
& +\frac{1}{\left(c \alpha^{0}\right)^{2}} \breve{g}^{i \rho} \breve{g}^{j \sigma} \breve{\delta}_{\nu}^{k} R[K]_{\rho \sigma}{ }^{\nu}{ }_{\omega} \breve{\delta}_{0}^{\omega} \partial_{i}^{0} \wedge \partial_{j}^{0} \wedge \partial_{k}^{0}
\end{aligned}
$$

and, in the adapted base (5.1), we have the coordinate expression

$$
\begin{aligned}
{[\Lambda, \Lambda]-\frac{2}{c^{2}} \gamma \wedge \Lambda=\frac{2}{c^{2}}\left[\frac{1}{2} \breve{g}^{j \rho} \breve{g}^{k \lambda} \nabla_{\rho} \hat{g}_{00}-\breve{g}^{k \rho} \breve{g}_{0 \lambda}\left(\nabla_{\rho} \breve{g}^{j \lambda}+\breve{g}^{j \sigma} K_{\rho}{ }^{\lambda}{ }\right)\right.} \\
\left.+\frac{1}{\left(\alpha^{0}\right)^{2}} \breve{g}^{k \rho}\left(\nabla_{\rho} \hat{g}^{i j}+\breve{\delta}_{\nu}^{i} \breve{g}^{j \sigma} K_{\rho}{ }^{\nu} \sigma+\hat{g}^{i j} \breve{\delta}_{0}^{\sigma} K_{\rho}{ }^{0} \sigma\right)\right] e_{i} \wedge e_{j}^{0} \wedge e_{k}^{0} \\
+\frac{2}{\left(c \alpha^{0}\right)^{2}} \breve{g}^{i \rho} \breve{g}^{j \sigma} \breve{\delta}_{\nu}^{k} \breve{\delta}_{0}^{\mu} R[K]_{\rho \sigma}{ }^{\nu}{ }_{\mu} e_{i}^{0} \wedge e_{j}^{0} \wedge e_{k}^{0} .
\end{aligned}
$$

Proof. It follows from (5.24), (5.25), (5.32) and (5.34).

6.25. Theorem. The pair $\left(-\frac{1}{c^{2}} \gamma, \Lambda\right)$ is a scaled Jacobi structure if and only if the condition (6.10) is satisfied.

Proof. It follows from Theorems 5.40 and 6.10 .

6.26. Corollary. In the particular case when $K$ is torsion free and the tensors $\nabla g$ and $g \otimes \nabla g$ are symmetric, $\left(-\frac{1}{c^{2}} \gamma, \Lambda\right)$ is a scaled Jacobi structure.

Proof. It follows from Corollary 6.11 and Theorem 6.25.

We can summarize the main results of the previous two sections as follows.

6.27. Theorem. The following assertions are equivalent.

(1) $g(Z, Z) d_{K} g(X, Y)(Z)+\frac{1}{2} g(Z, Y)\left(\nabla_{X} g\right)(Z, Z)-\frac{1}{2} g(Z, X)\left(\nabla_{Y} g\right)(Z, Z)=0$;

(2) $\Omega=-c^{2} d \tau$, i.e. the pair $\left(-c^{2} \tau, \Omega\right)$ is a scaled contact pair;

(3) $\left[-\frac{1}{c^{2}} \gamma, \Lambda\right]=0,[\Lambda, \Lambda]=\frac{2}{c^{2}} \gamma \wedge \Lambda$, i.e. the pair $\left(-\frac{1}{c^{2}} \gamma, \Lambda\right)$ is a scaled Jacobi pair.

Proof. It follows immediately from Theorems 6.19 and 6.25.

\section{MetriC AND NON-METRiC PHASE OBJECTS}

So far, we have analysed the scaled phase 2 -form $\Omega$ and the scaled phase 2 -vector $\Lambda$ associated with the metric $g$ and a generic phase connection $\Gamma$. 
On the other hand, the metric $g$ yields the Levi-Civita spacetime connection $K=: K[g]$, hence the distinguished metric phase connection $\Gamma[g]=: \chi(K[g])$. Accordingly, we obtain the distinguished scaled phase 2 -form $\Omega[g]=: \Omega[g, \Gamma[g]]$, the scaled phase 2-vector $\Lambda[g]=: \Lambda[g, \Gamma[g]]$ and the dynamical connection $\gamma[g]=: \gamma[\Gamma[g]]$.

Then, any phase connection $\Gamma$ and the associated scaled phase 2 -form $\Omega$ and scaled phase 2 -vector $\Lambda$ split into a metric component and an additional non-metric component.

This splitting provides further information on our phase structures. In particular, we shall see the effect of an additional closed spacetime 2-form, i.e. of an electromagnetic field.

7.1. Metric contact and Jacobi structures. We start by considering the metric scaled phase 2-form $\Omega[g]$ and scaled phase 2 -vector $\Lambda[g]$.

7.1. Theorem. The pairs $\left(-c^{2} \tau, \Omega[g]\right)$ and $\left(-\frac{1}{c^{2}} \gamma, \Lambda[g]\right)$ are a scaled contact structure and a scaled Jacobi structure, respectively, which are mutually dual.

Proof. The hypothesis $\nabla g=0$ and Corollary [6.20 imply that $\left(-c^{2} \tau, \Omega[g]\right)$ is a contact structure. On the other hand, we can also prove that $\Omega[g]=-d\left(c^{2} \tau\right)$, by a direct computation in coordinates.

Analogously, the hypothesis $\nabla g=0$ and Corollary [6.26 prove that $\left(-\frac{1}{c^{2}} \gamma, \Lambda[g]\right)$ is a Jacobi structure.

7.2. Non-metric contact and Jacobi structures. Next, we consider any phase connection $\Gamma$ and the induced scaled 2-form $\Omega=: \Omega[g, \Gamma]$, scaled 2-vector $\Lambda=: \Lambda[g, \Gamma]$ and dynamical connection $\gamma=: \gamma[\Gamma]$.

Here, we perform a further analysis of the conditions by which the pairs $\left(-c^{2} \tau, \Omega\right)$ and $\left(\frac{1}{c^{2}} \gamma, \Lambda\right)$ are a scaled contact structure and a scaled Jacobi structure, respectively.

In virtue of Theorems 5.41 and 6.27, the conditions for the contact structure and for the Jacobi structure coincide, for any phase connection $\Gamma$. For this reason, it suffices to prove explicitly only the conditions for the contact structure, which give the corresponding dual Jacobi structure, at the same time.

7.2. Proposition. Any phase connection $\Gamma$ can be uniquely written as

$$
\Gamma=\Gamma[g]+\Sigma, \quad \text { with } \quad \Sigma: \mathcal{J}_{1} \boldsymbol{E} \rightarrow T^{*} \boldsymbol{E} \otimes V \mathcal{J}_{1} \boldsymbol{E} .
$$

i.e., in coordinates, as

$$
\Gamma_{\lambda_{0}}^{i}=\Gamma[g]_{\lambda_{0}}^{i}+\Sigma_{\lambda_{0}}^{i}, \quad \text { with } \quad \Sigma_{\lambda_{0}}^{i} \in \operatorname{map}\left(\mathcal{J}_{1} \boldsymbol{E}, \mathbb{R}\right) .
$$

Correspondingly, the scaled phase 2 -form $\Omega$ and the scaled phase 2 -vector $\Lambda$ split as

$$
\Omega=: \Omega[g, \Gamma]=\Omega[g]+\Omega^{\mathfrak{a}}[g, \Sigma] \quad \text { and } \quad \Lambda=: \Lambda[g, \Gamma]=\Lambda[g]+\Lambda^{\mathfrak{a}}[g, \Sigma],
$$

where

$$
\begin{aligned}
& \left.\Omega^{\mathfrak{a}}[g, \Sigma]=: g\right\lrcorner\left(\left(\nu_{\tau} \circ \Sigma\right) \wedge \theta\right): \mathcal{J}_{1} \boldsymbol{E} \rightarrow\left(\mathbb{T}^{*} \otimes \mathbb{L}^{2}\right) \otimes \Lambda^{2} T^{*} \boldsymbol{E}, \\
& \left.\Lambda^{\mathfrak{a}}[g, \Sigma]=: \bar{g}\right\lrcorner\left(\Sigma \wedge \nu_{\tau}\right) \quad: \mathcal{J}_{1} \boldsymbol{E} \rightarrow\left(\mathbb{T} \otimes \mathbb{L}^{-2}\right) \otimes \Lambda^{2} V \mathcal{J}_{1} \boldsymbol{E},
\end{aligned}
$$


have the coordinate expressions

$$
\Omega^{\mathfrak{a}}[g, \Sigma]=-c \alpha^{0} \breve{g}_{i \mu} \Sigma_{\lambda_{0}}^{i} d^{\lambda} \wedge d^{\mu} \quad \text { and } \quad \Lambda^{\mathfrak{a}}[g, \Sigma]=\frac{1}{c \alpha^{0}} \breve{g}^{j \lambda} \Sigma_{\lambda_{0}}^{i} \partial_{i}^{0} \wedge \partial_{j}^{0} .
$$

Moreover, we have

$$
-c^{2} \tau \wedge \Omega[g, \Gamma] \wedge \Omega[g, \Gamma] \wedge \Omega[g, \Gamma]=-c^{2} \tau \wedge \Omega[g] \wedge \Omega[g] \wedge \Omega[g] .
$$

7.3. Lemma. Let us define the scaled $(0,2)$-tensor

$$
\underline{\Sigma}=:\left(g^{b} \circ \nu_{\tau}^{-1}\right)(\Sigma): \mathcal{J}_{1} \boldsymbol{E} \rightarrow\left(\mathbb{T}^{*} \otimes \mathbb{L}^{2}\right) \otimes T^{*} \boldsymbol{E} \otimes T^{*} \boldsymbol{E},
$$

with coordinate expression

$$
\underline{\Sigma}=c \alpha^{0} \breve{g}_{i \mu} \Sigma_{\lambda 0}^{i} d^{\lambda} \otimes d^{\mu}
$$

Indeed, we have

$$
\Omega^{\mathfrak{a}}[g, \Sigma]=-\operatorname{Alt} \underline{\Sigma} .
$$

Hence, the following conditions are equivalent:
1) $\Omega[g, \Gamma]=\Omega[g]$;
2) $\Omega^{\mathfrak{a}}[g, \Sigma]=0$;
3) $\underline{\Sigma}$ is symmetric.

Proof. The equality $\Omega^{\mathfrak{a}}[g, \Sigma]=-$ Alt $\underline{\Sigma}$ follows from the coordinate expressions of $\Omega^{\mathfrak{a}}[g, \Sigma]$ and Alt $\underline{\Sigma}$. Then, this equality implies the equivalence of conditions 1), 2) and 3).

7.4. Lemma. The scaled 2-form $L_{\Sigma} \tau: \mathcal{J}_{1} \boldsymbol{E} \rightarrow \mathbb{T} \otimes \Lambda^{2} T^{*} \boldsymbol{E}$ fulfills the equality

$$
L_{\Sigma} \tau=\frac{1}{c^{2}} \Omega^{\mathfrak{a}}[g, \Sigma]=-\frac{1}{c^{2}} \text { Alt } \underline{\Sigma} .
$$

Proof. The equalities $d \tau=-\frac{1}{c^{2}} \Omega[g]$ and $i_{\Sigma} \tau=0$ imply

$$
L_{\Sigma} \tau=i_{\Sigma} d \tau-d i_{\Sigma} \tau=\frac{1}{c^{2}} i_{\Sigma} \Omega[g]
$$

i.e., in coordinates,

$$
L_{\Sigma} \tau=-\frac{\alpha^{0}}{c} \breve{g}_{i \mu} \Sigma_{\lambda_{0}}^{i} d^{\lambda} \wedge d^{\mu}=\frac{1}{c^{2}} \Omega^{\mathfrak{a}}[g, \Sigma] .
$$

7.5. Theorem. The pairs $\left(-c^{2} \tau, \Omega\right)$ and $\left(-\frac{1}{c^{2}} \gamma, \Lambda\right)$ are scaled contact and Jacobi structures if and only if $\underline{\Sigma}$ is symmetric.

Proof. 1st Proof. In virtue of Theorem [7.1, we have $\Omega[g]=-c^{2} d \tau$. Hence, we obtain $\Omega=-c^{2} d \tau$ if and only if $\Omega^{\mathfrak{a}}[g, \Sigma]=0$, i.e., in virtue of Lemma [7.3, if and only if $\underline{\Sigma}$ is symmetric.

2nd Proof. Theorem 5.35 says that the pair $\left(-c^{2} \tau, \Omega[g, \Gamma]\right)$ is a scaled contact structure if and only if $L_{\Gamma} \tau=0$. On the other hand, in virtue of Corollary 6.11, we have $L_{\Gamma[g]} \tau=0$. Hence, the result follows from Lemma 7.4.

7.6. Note. Let $\Gamma$ and $\Gamma^{\prime}$ be two phase connections. Then, we have $\Omega[g, \Gamma]=\Omega\left[g, \Gamma^{\prime}\right]$, respectively $\Lambda[g, \Gamma]=\Lambda\left[g, \Gamma^{\prime}\right]$, if and only if the difference tensor $\Sigma=: \Gamma-\Gamma^{\prime}: \mathcal{J}_{1} \boldsymbol{E} \rightarrow$ $T^{*} \boldsymbol{E} \otimes V \mathcal{\partial}_{1} \boldsymbol{E}$ is such that $\Sigma=: g^{b}(\underline{\Phi})$ is symmetric.

Thus, the relations $\Omega[g, \Gamma]=\Omega\left[g, \Gamma^{\prime}\right]$, respectively $\Lambda[g, \Gamma]=\Lambda\left[g, \Gamma^{\prime}\right]$, define an equivalence relation on the space of phase connections. 
We are mainly interested in the phase connections which are equivalent to $\Gamma[g]$, because they yield an exact phase 2 -form $\Omega$.

7.7. Example. Let us consider the vertical valued 1 -form

$$
\Sigma=: \frac{c^{2} m}{\hbar} \nu_{\tau}: \mathcal{J}_{1} \boldsymbol{E} \rightarrow T^{*} \boldsymbol{E} \otimes V \mathcal{J}_{1} \boldsymbol{E},
$$

with coordinate expression

$$
\Sigma=\frac{c m}{\hbar \alpha^{0}} \breve{\delta}_{\mu}^{i} d^{\mu} \otimes \partial_{i}^{0} .
$$

Then, we obtain the symmetric scaled $(0,2)$-tensor

$$
\underline{\Sigma}=\frac{c^{2} m}{\hbar} g_{\perp}: \partial_{1} \boldsymbol{E} \rightarrow\left(\mathbb{T}^{*} \otimes \mathbb{L}^{2}\right) \otimes T^{*} \boldsymbol{E} \otimes T^{*} \boldsymbol{E},
$$

with coordinate expression

$$
\underline{\Sigma}=\frac{c^{2} m}{\hbar} \breve{g}_{i \mu} \breve{\delta}_{\lambda}^{i} d^{\lambda} \otimes d^{\mu}=\frac{c^{2} m}{\hbar}\left(g_{\mu \lambda}+\left(\alpha^{0}\right)^{2} \breve{g}_{0 \mu} \breve{g}_{0 \lambda}\right) d^{\lambda} \otimes d^{\mu}
$$

and the vanishing scaled phase 2 -form and 2 -vector

$$
\begin{gathered}
\Omega^{\mathfrak{a}}[g, \Sigma]: \mathcal{J}_{1} \boldsymbol{E} \rightarrow\left(\mathbb{T}^{*} \otimes \mathbb{L}^{2}\right) \otimes \Lambda^{2} T^{*} \boldsymbol{E}, \\
\Lambda^{\mathfrak{a}}[g, \Sigma]: \mathcal{J}_{1} \boldsymbol{E} \rightarrow\left(\mathbb{T} \otimes \mathbb{L}^{-2}\right) \otimes \Lambda^{2} T \boldsymbol{E},
\end{gathered}
$$

with coordinate expressions

$$
\begin{array}{ll}
\Omega^{\mathfrak{a}}[g, \Sigma]=-\frac{c^{2} m}{\hbar} \breve{g}_{i \mu} \breve{\delta}_{\lambda}^{i} d^{\lambda} \wedge d^{\mu}=-\frac{c^{2} m}{\hbar}\left(g_{\mu \lambda}+\left(\alpha^{0}\right)^{2} \breve{g}_{0 \mu} \breve{g}_{0 \lambda}\right) d^{\lambda} \wedge d^{\mu}=0, \\
\Lambda^{\mathfrak{a}}[g, \Sigma]=\frac{m}{\hbar\left(\alpha^{0}\right)^{2}} \breve{g}^{j \lambda} \breve{\delta}_{\lambda}^{i} \partial_{i}^{0} \wedge \partial_{j}^{0}=\frac{m}{\hbar\left(\alpha^{0}\right)^{2}} \hat{g}^{i j} \partial_{i}^{0} \wedge \partial_{j}^{0} & =0 .
\end{array}
$$

Thus, in virtue of Theorem $\left[7.5\right.$, the induced pairs $\left(-c^{2} \tau, \Omega[g, \Gamma]\right)$ and $\left(-\frac{1}{c^{2}} \gamma, \Lambda[g, \Gamma]\right)$ turn out to be scaled contact and Jacobi structures, respectively.

7.8. Lemma. Let us consider a scaled (0,3)-tensor

$$
\phi: \boldsymbol{E} \rightarrow \mathbb{L}^{2} \otimes\left(T^{*} \boldsymbol{E} \otimes T^{*} \boldsymbol{E} \otimes T^{*} \boldsymbol{E}\right)
$$

and the induced linear spacetime vertical valued 1 -form

$$
\Phi=: \Phi[g, \phi]=:\left(v \circ g^{\sharp 2}\right)(\phi): T \boldsymbol{E} \rightarrow T^{*} \boldsymbol{E} \otimes V T \boldsymbol{E},
$$

with coordinate expression

$$
\Phi=g^{\nu \rho} \phi_{\lambda \rho \mu} \dot{x}^{\mu} d^{\lambda} \otimes \dot{\partial}_{\nu} .
$$

Then, we obtain the linear spacetime connection $K=: K[g, \phi]=: K[g]+\Phi$, hence the phase connection $\Gamma=: \Gamma[g, \phi]=: \chi(K)$, which splits as $\Gamma=\Gamma[g]+\Sigma[g, \phi]$, where the vertical valued 1 -form

$$
\Sigma=: \Sigma[g, \phi]: \mathcal{J}_{1} \boldsymbol{E} \rightarrow T^{*} \boldsymbol{E} \otimes V \mathcal{J}_{1} \boldsymbol{E}
$$

has the coordinate expression

$$
\Sigma=\left(\breve{g}^{i \rho} \phi_{\lambda \rho \sigma} \breve{\delta}_{0}^{\sigma}\right) d^{\lambda} \otimes \partial_{i}^{0} .
$$


Thus, we obtain the associated scaled phase 2-form and 2-vector

$$
\Omega=: \Omega[g, \Gamma]=\Omega[g]+\Omega^{\mathfrak{a}}[g, \Sigma] \quad \text { and } \quad \Lambda=: \Lambda[g, \Gamma]=\Lambda[g]+\Lambda^{\mathfrak{a}}[g, \Sigma] .
$$

Indeed, the pairs $\left(-c^{2} \tau, \Omega\right)$ and $\left(-\frac{1}{c^{2}} \gamma, \Lambda\right)$ are scaled contact and Jacobi structures if and only if $L_{\Gamma} \tau=L_{\Sigma} \tau=0$, i.e., by Theorem 6.10, if and only if the condition (6.10) is satisfied, i.e. if and only if, for each $X, Y, Z \in \sec (\boldsymbol{E}, T \boldsymbol{E})$,

$g(Z, Z) \phi(X, Y, Z)-g(Z, Z) \phi(Y, X, Z)+g(Z, X) \phi(Y, Z, Z)-g(Z, Y) \phi(X, Z, Z)=0$.

7.3. Further examples of non-metric phase structures. Let us discuss further distinguished examples of non-metric phase structures generated by a horizontal scaled phase $(0,2)$-tensor. In the cases when this tensor is symmetric or antisymmetric we obtain different results.

Let us start with the general case.

7.9. Lemma. Given a scaled (0,2)-tensor

$$
\sigma: \mathcal{J}_{1} \boldsymbol{E} \rightarrow\left(\mathbb{T}^{*} \otimes \mathbb{L}^{2}\right) \otimes T^{*} \boldsymbol{E} \otimes T^{*} \boldsymbol{E}
$$

we obtain the vertical valued 1 -form

$$
\Sigma=: \Sigma[g, \sigma]=:\left(\nu_{\tau} \circ g^{\sharp 2}\right)(\sigma): \mathcal{J}_{1} \boldsymbol{E} \rightarrow T^{*} \boldsymbol{E} \otimes V \mathcal{J}_{1} \boldsymbol{E},
$$

with coordinate expression

$$
\Sigma=\frac{1}{c \alpha^{0}} \breve{g}^{i \rho} \sigma_{\lambda \rho} d^{\lambda} \otimes \partial_{i}^{0} .
$$

Hence, according to Proposition 7.2, we obtain the phase connection

$$
\Gamma=: \Gamma[g, \sigma]=: \Gamma[g]+\Sigma[g, \sigma]
$$

and the associated scaled phase 2 -form and 2 -vector

$$
\begin{aligned}
& \Omega=: \Omega[g, \sigma]=: \Omega[g, \Gamma]=\Omega[g]+\Omega^{\mathfrak{a}}[g, \sigma] \\
& \Lambda=: \Lambda[g, \sigma]=: \Lambda[g, \Gamma]=\Lambda[g]+\Lambda^{\mathfrak{a}}[g, \sigma] .
\end{aligned}
$$

We have

$$
\begin{array}{rlrl}
\Omega^{\mathfrak{a}}[g, \sigma] & =-\operatorname{Alt}\left(\pi_{\perp}^{2}(\sigma)\right) & : \mathcal{J}_{1} \boldsymbol{E} \rightarrow\left(\mathbb{T}^{*} \otimes \mathbb{L}^{2}\right) \otimes \Lambda^{2} T^{*} \boldsymbol{E}, \\
\Lambda^{\mathfrak{a}}[g, \sigma] & =-\operatorname{Alt}\left(\left(\nu_{\tau} \circ g^{\sharp}\right) \otimes\left(\nu_{\tau} \circ g^{\sharp}\right)\right)(\sigma): \mathcal{J}_{1} \boldsymbol{E} \rightarrow\left(\mathbb{T}^{*} \otimes \mathbb{L}^{2}\right) \otimes \Lambda^{2} T^{*} \boldsymbol{E}
\end{array}
$$

(where $\pi_{\perp}^{2}$ denotes the orthogonal projection of the 2 nd factor), i.e., in coordinates,

$$
\Omega^{\mathfrak{a}}[g, \sigma]=-\breve{g}_{i \mu} \breve{g}^{i \rho} \sigma_{\lambda \rho} d^{\lambda} \wedge d^{\mu} \quad \text { and } \quad \Lambda^{\mathfrak{a}}[g, \sigma]=-\frac{1}{\left(c \alpha^{0}\right)^{2}} \breve{g}^{i \lambda} \breve{g}^{j \mu} \sigma_{\lambda \mu} \partial_{i}^{0} \wedge \partial_{j}^{0}
$$

Moreover, we define the scaled $(0,2)$-tensor

$$
\left.[\sigma]=: \sigma-(\text { д }\lrcorner^{2} \sigma\right) \otimes \tau: \mathcal{J}_{1} \boldsymbol{E} \rightarrow\left(\mathbb{T}^{*} \otimes \mathbb{L}^{2}\right) \otimes T^{*} \boldsymbol{E} \otimes T^{*} \boldsymbol{E}
$$

(where $\lrcorner^{2}$ denotes the insertion on the 2 nd factor), with coordinate expression

$$
[\sigma]=\left(\sigma_{\lambda \mu}+\left(\alpha^{0}\right)^{2} \sigma_{\lambda \rho} \breve{\delta}_{0}^{\rho} \breve{g}_{0 \mu}\right) d^{\lambda} \otimes d^{\mu} .
$$

Indeed, we have $L_{\Gamma} \tau=0$ if and only if $[\sigma]$ is symmetric. 
Proof. We have $L_{\Gamma} \tau=L_{\Gamma[g]} \tau+L_{\Sigma} \tau=L_{\Sigma} \tau=\left(\sigma_{\lambda \mu}+\left(\alpha^{0}\right)^{2} \breve{g}_{0 \mu} \sigma_{\lambda \rho} \breve{\delta}_{0}^{\rho}\right) d^{\lambda} \wedge d^{\mu}$.

Hence, $L_{\Gamma} \tau=0$ if and only if $[\sigma]$ is symmetric.

7.10. Theorem. The pairs $\left(-c^{2} \tau, \Omega[g, \sigma]\right)$ and $\left(-\frac{1}{c^{2}} \gamma, \Lambda[g, \sigma]\right)$ are scaled contact and Jacobi structures if and only if $[\sigma]$ is symmetric.

7.11. Example. Let us consider the scaled $(0,2)$-tensor

$$
\sigma=: \frac{c^{2} m}{\hbar}\left(g+k c^{2} \tau \otimes \tau\right)
$$

with $k \in \operatorname{map}\left(\mathcal{J}_{1} \boldsymbol{E}, \mathbb{R}\right)$, and the induced vertical valued form and the scaled 2-form

$$
\Sigma=:\left(\nu_{\tau} \circ g^{\sharp 2}\right)(\sigma)=\frac{c^{2} m}{\hbar} \nu_{\tau} .
$$

Indeed, we obtain the symmetric scaled $(0,2)$-tensor (which does not depend on $k$ )

$$
[\sigma]=\frac{c^{2} m}{\hbar}\left(g+c^{2} \tau \otimes \tau\right)
$$

and the scaled phase 2-form and 2-vector

$$
\begin{aligned}
\Omega^{\mathfrak{a}}[g, \sigma] & =-\frac{c^{2} m}{\hbar} \breve{g}_{i \mu} \breve{g}^{i \rho} g_{\lambda \rho} d^{\lambda} \wedge d^{\mu} \\
& =-\frac{c^{2} m}{\hbar}\left(\delta_{\mu}^{\rho}+\left(\alpha^{0}\right)^{2} \breve{g}_{0 \mu} \breve{\delta}_{0}^{\rho}\right) g_{\lambda \rho} d^{\lambda} \wedge d^{\mu} \\
& =-\frac{c^{2} m}{\hbar}\left(g_{\lambda \mu}+\left(\alpha^{0}\right)^{2} \breve{g}_{0 \lambda} \breve{g}_{0 \mu}\right) d^{\lambda} \wedge d^{\mu}=0, \\
\Lambda^{\mathfrak{a}}[g, \sigma] & =-\frac{1}{\left(c \alpha^{0}\right)^{2}} \breve{g}^{i \lambda} \breve{g}^{j \mu} g_{\lambda \mu} \partial_{i}^{0} \wedge \partial_{j}^{0}=0 .
\end{aligned}
$$

Thus, in virtue of Theorem 7.10 and according to Example 7.7 , the pairs $\left(-c^{2} \tau, \Omega[g, \sigma]\right)$ and $\left(-\frac{1}{c^{2}} \gamma, \Lambda[g, \sigma]\right)$ are scaled contact and Jacobi structures.

7.12. Lemma. Let us consider a scaled symmetric $(0,2)$-tensor

$$
\psi: \mathcal{J}_{1} \boldsymbol{E} \rightarrow\left(\mathbb{T}^{*} \otimes \mathbb{L}^{2}\right) \otimes S^{2} T^{*} \boldsymbol{E}
$$

and the induced scaled symmetric $(0,2)$-tensor

$$
\left.\sigma=:-\frac{1}{2}(\psi-2(\text { д }\lrcorner) \odot \tau\right): \partial_{1} \boldsymbol{E} \rightarrow\left(\mathbb{T}^{*} \otimes \mathbb{L}^{2}\right) \otimes S^{2} T^{*} \boldsymbol{E},
$$

with coordinate expression

$$
\sigma=-\frac{1}{2}\left(\psi_{\lambda \mu}+2\left(\alpha^{0}\right)^{2} \breve{g}_{0 \lambda}\left(\psi_{0 \mu}+x_{0}^{i} \psi_{i \mu}\right)\right) d^{\lambda} \odot d^{\mu} .
$$

Then, the induced vertical valued 1 -form turns out to be the section

$$
\left.\Sigma=-\frac{1}{2}\left(\nu_{\tau} \circ g^{\sharp 2}\right)(\psi-\tau \otimes(\text { д }\lrcorner \psi)\right),
$$

with coordinate expression

$$
\Sigma=\frac{1}{2 c \alpha^{0}} \breve{g}^{i \rho}\left(\psi_{\lambda \rho}+\left(\alpha^{0}\right)^{2} \breve{g}_{0 \lambda}\left(\psi_{0 \rho}+\psi_{i \rho} x_{0}^{i}\right)\right) d^{\lambda} \otimes \partial_{i}^{0} .
$$


Indeed, the scaled $(0,2)$-tensor

$$
\left.\left.\left.[\sigma]=-\frac{1}{2}(\psi-2 \tau \odot(\text { д }\lrcorner \psi)+(\text { д }\lrcorner \text { д }\right\lrcorner \psi\right) \tau \otimes \tau\right)
$$

turns out to be symmetric. Hence, in virtue of Theorem 7.10 , we have

$$
\Omega^{\mathfrak{a}}[g, \sigma]=0=\Lambda^{\mathfrak{a}}[g, \sigma]
$$

and the pairs $\left(-c^{2} \tau, \Omega[g, \sigma]\right)$ and $\left(-\frac{1}{c^{2}} \gamma, \Lambda[g, \sigma]\right)$ are scaled contact and Jacobi structures, respectively.

7.13. Note. With reference to the above Lemma, a direct computation gives

$$
\begin{aligned}
\Omega^{\mathfrak{a}}[g, \sigma]= & -c \alpha^{0} \breve{g}_{i \mu} \Sigma_{\lambda_{0}}^{i} d^{\lambda} \wedge d^{\mu} \\
= & -\frac{1}{2} \breve{g}_{i \mu} \breve{g}^{i \rho}\left(\psi_{\lambda \rho}+\left(\alpha^{0}\right)^{2} \breve{g}_{0 \lambda}\left(\psi_{0 \rho}+x_{0}^{i} \psi_{i \rho}\right)+\left(\alpha^{0}\right)^{2} \breve{g}_{0 \rho}\left(\psi_{0 \lambda}+x_{0}^{i} \psi_{i \lambda}\right)\right) d^{\lambda} \wedge d^{\mu} \\
= & -\frac{1}{2}\left(\delta_{\mu}^{\rho}+\left(\alpha^{0}\right)^{2} \breve{g}_{0 \mu} \breve{\delta}_{0}^{\rho}\right)\left(\psi_{\lambda \rho}+\left(\alpha^{0}\right)^{2}\left(\breve{g}_{0 \lambda} \psi_{\sigma \rho}+\breve{g}_{0 \rho} \psi_{\sigma \lambda}\right) \breve{\delta}_{0}^{\sigma}\right) d^{\lambda} \wedge d^{\mu} \\
= & -\frac{1}{2}\left(\psi_{\lambda \mu}+\left(\alpha^{0}\right)^{2}\left(\breve{g}_{0 \lambda} \psi_{\sigma \mu}+\breve{g}_{0 \mu} \psi_{\sigma \lambda}\right) \breve{\delta}_{0}^{\sigma}+\left(\alpha^{0}\right)^{2} \breve{g}_{0 \mu} \psi_{\lambda \rho} \breve{\delta}_{0}^{\rho}\right. \\
& \left.\quad+\left(\alpha^{0}\right)^{4}\left(\breve{g}_{0 \lambda} \breve{g}_{0 \mu} \psi_{\sigma \rho} \breve{\delta}_{0}^{\rho} \breve{\delta}_{0}^{\sigma}+\breve{g}_{0 \rho} \breve{g}_{0 \mu} \psi_{\sigma \lambda} \breve{\delta}_{0}^{\rho} \breve{\delta}_{0}^{\sigma}\right)\right) d^{\lambda} \wedge d^{\mu} \\
= & -\frac{1}{2}\left(\psi_{\lambda \mu}+\left(\alpha^{0}\right)^{2}\left(\breve{g}_{0 \lambda} \psi_{\sigma \mu}+\breve{g}_{0 \mu} \psi_{\sigma \lambda}\right) \breve{\delta}_{0}^{\sigma}+\left(\alpha^{0}\right)^{2} \breve{g}_{0 \mu} \psi_{\lambda \rho} \breve{\delta}_{0}^{\rho}\right. \\
\quad & \left.\left.\quad+\left(\alpha^{0}\right)^{4} \breve{g}_{0 \lambda} \breve{g}_{0 \mu} \psi_{\sigma \rho} \breve{\delta}_{0}^{\rho} \breve{\delta}_{0}^{\sigma}-\left(\alpha^{0}\right)^{2} \breve{g}_{0 \mu} \psi_{\sigma \lambda} \breve{\delta}_{0}^{\sigma}\right)\right) d^{\lambda} \wedge d^{\mu} \\
= & 0 . \quad
\end{aligned}
$$

7.14. Lemma. Let us consider a scaled antisymmetric $(0,2)$-tensor

$$
\varphi: \mathcal{J}_{1} \boldsymbol{E} \rightarrow\left(\mathbb{T}^{*} \otimes \mathbb{L}^{2}\right) \otimes \Lambda^{2} T^{*} \boldsymbol{E},
$$

and the induced scaled antisymmetric (0,2)-tensor

$$
\left.\sigma=:-\frac{1}{2}(\varphi-2(\text { д }\lrcorner) \wedge \tau\right): \mathcal{J}_{1} \boldsymbol{E} \rightarrow\left(\mathbb{T}^{*} \otimes \mathbb{L}^{2}\right) \otimes \Lambda^{2} T^{*} \boldsymbol{E},
$$

with coordinate expression

$$
\sigma=-\frac{1}{2}\left(\varphi_{\lambda \mu}-2\left(\alpha^{0}\right)^{2} \breve{g}_{0 \lambda} \varphi_{\sigma \mu} \breve{\delta}_{0}^{\sigma}\right) d^{\lambda} \wedge d^{\mu} .
$$

Then, the induced vertical valued 1 -form turns out to be the section

$$
\left.\Sigma=-\frac{1}{2}\left(\nu_{\tau} \circ g^{\sharp 2}\right)(\varphi+\tau \otimes(\text { д }\lrcorner \varphi)\right),
$$

with coordinate expression

$$
\Sigma=-\frac{1}{2} \frac{1}{c \alpha^{0}} \breve{g}^{i \rho}\left(\varphi_{\lambda \rho}-\left(\alpha^{0}\right)^{2} \breve{g}_{0 \lambda} \varphi_{\sigma \rho} \breve{\delta}_{0}^{\sigma}\right) d^{\lambda} \otimes \partial_{i}^{0} .
$$

Hence, the induced scaled phase 2 -form and $(0,2)$-vector turn out to be

$$
\Omega^{\mathfrak{a}}[g, \Sigma]=\frac{1}{2} \varphi \quad \text { and } \quad \Lambda^{\mathfrak{a}}[g, \Sigma]=\frac{1}{2} \operatorname{Alt}\left(\left(\nu_{\tau} \circ g^{\sharp}\right) \otimes\left(\nu_{\tau} \circ g^{\sharp}\right)\right)(\varphi) .
$$

Proof. 1st proof. We have

$$
\begin{aligned}
\Omega^{\mathfrak{a}}[g, \Sigma] & =-c \alpha^{0} \breve{g}_{i \mu} \Sigma_{\lambda_{0}}^{i} d^{\lambda} \wedge d^{\mu} \\
& =\frac{1}{2} \breve{g}_{i \mu} \breve{g}^{i \rho}\left(\varphi_{\lambda \rho}-\left(\alpha^{0}\right)^{2}\left(\breve{g}_{0 \lambda} \varphi_{\sigma \rho}-\breve{g}_{0 \rho} \varphi_{\sigma \lambda}\right) \breve{\delta}_{0}^{\sigma}\right) d^{\lambda} \wedge d^{\mu} \\
& =\frac{1}{2}\left(\delta_{\mu}^{\rho}+\left(\alpha^{0}\right)^{2} \breve{g}_{0 \mu} \breve{\delta}_{0}^{\rho}\right)\left(\varphi_{\lambda \rho}-\left(\alpha^{0}\right)^{2}\left(\breve{g}_{0 \lambda} \varphi_{\sigma \rho}-\breve{g}_{0 \rho} \varphi_{\sigma \lambda}\right) \breve{\delta}_{0}^{\sigma}\right) d^{\lambda} \wedge d^{\mu}
\end{aligned}
$$




$$
\begin{aligned}
= & \frac{1}{2}\left(\varphi_{\lambda \mu}-\left(\alpha^{0}\right)^{2} \breve{g}_{0 \lambda} \varphi_{\sigma \mu} \breve{\delta}_{0}^{\sigma}+\left(\alpha^{0}\right)^{2} \breve{g}_{0 \mu} \varphi_{\sigma \lambda} \breve{\delta}_{0}^{\sigma}+\left(\alpha^{0}\right)^{2} \breve{g}_{0 \mu} \varphi_{\lambda \sigma}\right] \breve{\delta}_{0}^{\sigma} \\
& \left.-\left(\alpha^{0}\right)^{4} \breve{g}_{0 \lambda} \breve{g}_{0 \mu} \varphi_{\sigma \rho} \breve{\delta}_{0}^{\sigma} \breve{\delta}_{0}^{\rho}+\left(\alpha^{0}\right)^{4} \breve{g}_{0 \mu} \hat{g}_{00} \varphi_{\sigma \lambda} \breve{\delta}_{0}^{\sigma}\right) d^{\lambda} \wedge d^{\mu} \\
= & \frac{1}{2} \varphi_{\lambda \mu} d^{\lambda} \wedge d^{\mu}=\frac{1}{2} \varphi \\
\Lambda^{\mathfrak{a}}[g, \Sigma]= & \frac{1}{c \alpha^{0}} \breve{g}^{j \lambda} \Sigma_{\lambda_{0}}^{i} \partial_{i}^{0} \wedge \partial_{j}^{0} \\
= & -\frac{1}{2\left(c \alpha^{0}\right)^{2}} \breve{g}^{j \lambda} \breve{g}^{i \rho}\left(\varphi_{\lambda \rho}-\left(\alpha^{0}\right)^{2} \breve{g}_{0 \lambda} \varphi_{\sigma \rho} \breve{\delta}_{0}^{\sigma}\right) \partial_{i}^{0} \wedge \partial_{j}^{0} \\
= & -\frac{1}{2\left(c \alpha^{0}\right)^{2}} \breve{g}^{j \lambda} \breve{g}^{i \rho} \varphi_{\lambda \rho} \partial_{i}^{0} \wedge \partial_{j}^{0} .
\end{aligned}
$$

2nd proof. By applying Lemma 7.9 and recalling the identity д $\lrcorner \tau=1$, we obtain

$$
\begin{aligned}
\Omega^{\mathfrak{a}}[g, \Sigma]= & -\operatorname{Alt}\left(\pi_{\perp}^{2}(\sigma)\right) \\
= & \left.\frac{1}{2} \operatorname{Alt}\left(\pi_{\perp}^{2}(\varphi-2(\text { д }\lrcorner) \wedge \tau\right)\right) \\
= & \left.\left.\frac{1}{2} \operatorname{Alt}\left(\pi_{\perp}^{2}(\varphi+\tau \otimes(\text { д }\lrcorner)-(\text { д }\lrcorner \varphi\right) \otimes \tau\right)\right) \\
= & \left.\left.\frac{1}{2} \operatorname{Alt}(\varphi-(\text { д }\lrcorner) \otimes \tau+\tau \otimes(\text { д }\lrcorner\right)+(\text { д }\lrcorner \varphi\right) \otimes \tau \\
& -\tau \otimes(\text { Д }\lrcorner) \tau-(\text { д }\lrcorner\lrcorner \varphi) \tau \otimes \tau)=\frac{1}{2} \varphi, \\
\Lambda^{\mathfrak{a}}[g, \Sigma]= & -\operatorname{Alt}\left(\left(\nu_{\tau} \circ g^{\sharp}\right) \otimes\left(\nu_{\tau} \circ g^{\sharp}\right)\right)(\sigma) \\
= & \frac{1}{2} \operatorname{Alt}\left(\left(\nu_{\tau} \circ g^{\sharp}\right) \otimes\left(\nu_{\tau} \circ g^{\sharp}\right)\right)(\varphi) .
\end{aligned}
$$

In the above Lemmas, we have defined $\sigma$ by using a normalising factor $\frac{1}{2}$; indeed, this factor might be chosen in an arbitrary way. Our choice is selected in such a way that, in the case when the source of the additional term $\phi$ is the electromagnetic field, the resulting $\Omega^{\mathfrak{a}}$ and $\Lambda^{\mathfrak{a}}$ be normalised as it is usually done in the standard literature.

7.15. Theorem. Let us consider a scaled (0,2)-tensor

$$
\omega=\psi+\varphi: \mathcal{J}_{1} \boldsymbol{E} \rightarrow\left(\mathbb{T}^{*} \otimes \mathbb{L}^{2}\right) \otimes T^{*} \boldsymbol{E} \otimes T^{*} \boldsymbol{E},
$$

where $\psi$ and $\phi$ are the symmetric and antisymmetric components of $\omega$, respectively, and the induced scaled (0,2)-tensor

$$
\left.\left.\sigma=:-\frac{1}{2}(\omega-(\text { д }\lrcorner \omega) \otimes \tau-\tau \otimes(\text { д }\lrcorner^{2} \omega\right)\right): \partial_{1} \boldsymbol{E} \rightarrow\left(\mathbb{T}^{*} \otimes \mathbb{L}^{2}\right) \otimes T^{*} \boldsymbol{E} \otimes T^{*} \boldsymbol{E},
$$

with coordinate expression

$$
\sigma=-\frac{1}{2}\left(\omega_{\lambda \mu}+\left(\alpha^{0}\right)^{2} \breve{g}_{0 \mu}\left(\omega_{0 \lambda}+\omega_{i \lambda} x_{0}^{i}\right)+\left(\alpha^{0}\right)^{2} \breve{g}_{0 \lambda}\left(\omega_{\mu 0}+\omega_{\mu i} x_{0}^{i}\right)\right) d^{\lambda} \otimes d^{\mu} .
$$

Then, the induced vertical valued 1-form turns out to be the section

$$
\left.\Sigma[g, \sigma]=-\frac{1}{2}\left(\nu_{\tau} \circ g^{\sharp 2}\right)\left(\omega-\tau \otimes(\text { д }\lrcorner^{2} \omega\right)\right),
$$

with coordinate expression

$$
\left.\Sigma[g, \sigma]=-\frac{1}{2 c \alpha^{0}} \breve{g}^{i \rho}\left(\omega_{\lambda \rho}-\left(\alpha^{0}\right)^{2} \breve{g}_{0 \lambda} \omega_{\sigma \rho} \breve{\delta}_{0}^{\sigma}\right)\right) d^{\lambda} \otimes \partial_{i}^{0} .
$$

Hence, the induced scaled phase 2-form and 2-vector turn out to be

$$
\Omega[g, \Sigma]=-c^{2} d \tau+\frac{1}{2} \varphi \quad \text { and } \quad \Lambda[g, \Sigma]=\Lambda[g]+\frac{1}{2} \operatorname{Alt}\left(\left(\nu_{\tau} \circ g^{\sharp}\right) \otimes\left(\nu_{\tau} \circ g^{\sharp}\right)\right)(\varphi) \text {. }
$$


Proof. It follows from Lemma 7.12 and Lemma 7.14 .

7.16. Lemma. Let $A: \mathcal{J}_{1} \boldsymbol{E} \rightarrow\left(\mathbb{T}^{*} \otimes \mathbb{L}^{2}\right) \otimes T^{*} \boldsymbol{E}$ be a scaled 1 -form and $\Gamma=\Gamma[g]+\Sigma$ any phase connection. Then, we have

$$
\left(-c^{2} \tau+A\right) \wedge \Omega[g, \Gamma] \wedge \Omega[g, \Gamma] \wedge \Omega[g, \Gamma]=\left(-c^{2} \tau+A\right) \wedge \Omega[g] \wedge \Omega[g] \wedge \Omega[g] .
$$

Proof. We can easily see, in coordinates, that the terms including $\Omega^{\mathfrak{a}}[g, \Sigma]$ disappear in the wedge product because they are horizontal 2 -forms, hence they generate horizontal forms of degree greater than 4.

We stress that the above 7 -form $\left(-c^{2} \tau+A\right) \wedge \Omega[g] \wedge \Omega[g] \wedge \Omega[g]$ needs not to be a volume form.

7.17. Lemma. Let us consider an exact scaled 2-form

$$
\varphi=: 2 d A: \mathcal{J}_{1} \boldsymbol{E} \rightarrow\left(\mathbb{T}^{*} \otimes \mathbb{L}^{2}\right) \otimes \Lambda^{2} T^{*} \boldsymbol{E}
$$

and the induced phase 2 -form $\Omega[g, \varphi]=: \Omega[g]+\Omega^{\mathfrak{a}}[g, \varphi]$.

Then, we have

$$
\Omega[g, \varphi]=d\left(-c^{2} \tau+A\right)
$$

if and only if $A$ is the pullback of a scaled spacetime 1 -form $A: \boldsymbol{E} \rightarrow\left(\mathbb{T}^{*} \otimes \mathbb{L}^{2}\right) \otimes T^{*} \boldsymbol{E}$.

Proof. In fact, we have $\Omega[g, \varphi]=-c^{2} d \tau$ and

$$
\Omega^{\mathfrak{a}}[g, \phi]=-c \alpha^{0} \breve{g}_{i \mu} \Sigma_{\lambda 0}^{i} d^{\lambda} \wedge d^{\mu}=\partial_{\lambda} A_{\mu} d^{\lambda} \wedge d^{\mu}+\partial_{i}^{0} A_{\lambda} d_{0}^{i} \wedge d^{\lambda} .
$$

Hence, $\Omega^{\mathfrak{a}}[g, \varphi]=d A$ if and only if $\partial_{i}^{0} A_{\lambda}=0$.

We can summarize the above results as follows.

7.18. Corollary. Let us consider a closed scaled spacetime 2-form

$$
\varphi: \boldsymbol{E} \rightarrow\left(\mathbb{T}^{*} \otimes \mathbb{L}^{2}\right) \otimes \Lambda^{2} T^{*} \boldsymbol{E}
$$

and the induced phase 2 -form $\Omega[g, \varphi]=: \Omega[g]+\Omega^{\mathfrak{a}}[g, \varphi]=\Omega[g]+\frac{1}{2} \varphi$.

Then, the pair $\left(-c^{2} \tau, \Omega[g, \varphi]\right)$ turns out to be an almost-cosymplectic-contact structure, i.e. $\Omega[g, \varphi]$ is closed and $-c^{2} \tau \wedge \Omega \wedge \Omega \wedge \Omega$ is a volume form.

Moreover, if $\varphi=2 d A$, where

$$
A: \boldsymbol{E} \rightarrow\left(\mathbb{T}^{*} \otimes \mathbb{L}^{2}\right) \otimes T^{*} \boldsymbol{E}
$$

is a scaled spacetime 1 -form, then the induced phase 2 -form is given by $\Omega[g, 2 d A]=: \Omega[g]+$ $\Omega^{\mathfrak{a}}[g, 2 d A]=d\left(-c^{2} \tau+A\right)$.

Hence, the pair $\left(-c^{2} \tau+A, \Omega[g, 2 d A]\right)$ turns out to be a contact structure if and only if $\left(-c^{2} \tau+A\right) \wedge \Omega[g] \wedge \Omega[g] \wedge \Omega[g]$ is everywhere non vanishing.

7.19. Remark. Let us consider a closed 2-form $\varphi: \boldsymbol{E} \rightarrow\left(\mathbb{T}^{*} \otimes \mathbb{L}^{2}\right) \otimes \Lambda^{2} T^{*} \boldsymbol{E}$. Then, the dual almost-coPoisson-Jacobi pair $\left(-\frac{1}{c^{2}} \gamma[\varphi], \Lambda[g, \varphi]\right)$ is given by

$$
\gamma=\gamma[g]+\gamma[\varphi]=: \text { д }\lrcorner(\Gamma[g]+\Sigma[\varphi])
$$

and $\Lambda[g, \varphi]=: \Lambda[g]+\Lambda^{\mathfrak{a}}[g, \varphi]$, where

$$
\left.\Sigma=-\frac{1}{2}\left(\nu_{\tau} \circ g^{\sharp 2}\right)(\varphi+\tau \otimes(\text { д }\lrcorner \varphi)\right)
$$


and

$$
\Lambda^{\mathfrak{a}}[g, \Sigma]=\frac{1}{2} \operatorname{Alt}\left(\left(\nu_{\tau} \circ g^{\sharp}\right) \otimes\left(\nu_{\tau} \circ g^{\sharp}\right)\right)(\varphi) .
$$

We can apply the above results to the phase structures associated with an electromagnetic field represented by a scaled closed 2-form $F: \boldsymbol{E} \rightarrow\left(\mathbb{L}^{1 / 2} \otimes \mathbb{M}^{1 / 2}\right) \otimes \Lambda^{2} T^{*} \boldsymbol{E}$.

For this purpose, it suffices to consider a mass $m \in \mathbb{M}$ and a charge $q \in \mathbb{T}^{-1} \otimes \mathbb{L}^{3 / 2} \otimes \mathbb{M}^{1 / 2}$ and set $\varphi=: \frac{q}{m} F$.

\section{REFERENCES}

[1] M. De Leon, G.M. Tuynman: A universal model for cosymplectic manifolds, J. Geom. Phys. 20 (1996) 77-86.

[2] J. JANYŠKA: Remarks on symplectic and contact 2-forms in relativistic theories, Bollettino U.M.I. (7) 9-B (1995) 587-616.

[3] J. JANYŠKA: Natural Poisson and Jacobi structures on the tangent bundle of a pseudo-Riemannian manifold, Contemporary Mathematics 288, Global Diff. Geom.: The Math. Legacy of Alfred Gray, eds. M. Fernándes and J. A. Wolf, 2001, 343-347.

[4] J. JANYŠKA: Geometric structures on the tangent bundle of the Einstein spacetime, Arch. Math. (Brno). 42 (2006) 195-203, arXiv: math/0508449.

[5] J. JanYŠKa, M. Modugno: Classical particle phase space in general relativity, in: Differential Geometry and Applications, Proc. Conf., Aug. 28 - Sept. 1, 1995, Brno, Czech republic, Masaryk University, Brno 1996, 573-602.

[6] J. JanyšKa, M. Modugno: Relations between linear connections on the tangent bundle and connections on the jet bundle of a fibred manifold, Arch. Math. (Brno) 32 (1996) 281-288, in honour of I. Kolár̆.

[7] J. JANYŠKa J., M. Modugno: Hermitian vector fields and special phase functions, Internat. J. Geom. Methods in Modern Physics 3 (2006) 719-754, arXiv: math-ph/0507070.

[8] J. JanyšKa, M. Modugno: Generalized geometrical structures of odd dimensional manifolds, arXiv: 0801.1447.

[9] J. JanyšKa, M. Modugno, R. Vitolo: Semi-vector spaces and units of measurement, arXiv: 0710.1313.

[10] I. Kolár̆, P. W. Michor, J. Slovák: Natural Operations in Differential Geometry, SpringerVerlag 1993.

[11] P. Libermann, Ch. M. Marle: Symplectic Geometry and Analytical Mechanics, Reidel Publ., Dordrecht 1987.

[12] I. Vaisman: Lectures on the Geometry of Poisson Manifolds, Birkhäuser Verlag 1994.

[13] A. M. Vinogradov: An informal introduction to the geometry of jet spaces, Rend. Seminari Fac. Sci. Univ. Cagliari 58 (1988) 301-333.

[14] H. WeYL: Zur Infinitesimalgeometrie; Einordnung der projektiven und der knoformen Auffassung, Gottingen Nachr. (1921) 99-122.

Department of Mathematics and Statistics, Masaryk University JanáČKovo nám 2A, 60200 BRno, CZeCh RePublic

E-MAIL: janyska@math.muni.cz

Department of Applied Mathematics, Florence University

Via S. Marta 3, 50139 Florence, Italy

E-MAIL: marco.modugno@unifi.it 